\title{
On the Impact of Known-Key Attacks on Hash Functions
}

\author{
Bart Mennink and Bart Preneel \\ Dept. Electrical Engineering, ESAT/COSIC, KU Leuven, and iMinds, Belgium \\ bart.mennink@esat.kuleuven.be, bart.preneel@esat.kuleuven.be
}

\begin{abstract}
Hash functions are often constructed based on permutations or blockciphers, and security proofs are typically done in the ideal permutation or cipher model. However, once these random primitives are instantiated, vulnerabilities of these instantiations may nullify the security. At ASIACRYPT 2007, Knudsen and Rijmen introduced known-key security of blockciphers, which gave rise to many distinguishing attacks on existing blockcipher constructions. In this work, we analyze the impact of such attacks on primitive-based hash functions. We present and formalize the weak cipher model, which captures the case a blockcipher has a certain weakness but is perfectly random otherwise. A specific instance of this model, considering the existence of sets of $B$ queries whose XOR equals 0 at bit-positions $C$, where $C$ is an index set, covers a wide range of known-key attacks in literature. We apply this instance to the PGV compression functions, as well as to the Grøstl (based on two permutations) and Shrimpton-Stam (based on three permutations) compression functions, and show that these designs do not seriously succumb to any differential known-key attack known to date.
\end{abstract}

Keywords. Hash functions, known-key security, Knudsen-Rijmen, PGV, Grøstl, Shrimpton-Stam, collision resistance, preimage resistance.

\section{Introduction}

Cryptographic hash functions are conventionally built on top of compression functions, and in turn on one or more blockciphers. Since the first appearance of such compression function $\mathrm{F}(h, m)=\operatorname{DES}_{m}(h)$ by Rabin 49 in the late 70s, many blockcipher-based functions appeared in the literature $23,25,29,30,40,43,48,59$. These all enjoy security proofs in the ideal model, where the underlying ciphers are assumed to behave ideally. Characteristic to these designs is that the key input to the cipher depends on the input to the compression function, and that the key scheduling needs to be sufficiently strong. For instance, Biryukov et al. 6 derived a related-key attack on AES and claimed that it invalidates the security of the Davies-Meyer compression function when the underlying primitive is instantiated with AES. A more recent approach to compression function design is to base them on a limited number of permutations 8, 41, 42, 51, 57. These permutations could be designed from scratch, or obtained by fixing a small set of keys and using a blockcipher for these keys only. Related- or chosen-key attacks on blockciphers do not help the adversary here, as the keys are fixed.

Known-Key Security of Blockciphers. While in the classical security models for blockciphers the key is secret and randomly drawn and the adversary's target is to distinguish the instantiation of the cipher from a random permutation (also known as (strong) pseudorandom permutation security), this notion does not apply if the key is known to the adversary. At ASIACRYPT 2007, Knudsen and Rijmen 27] introduced known-key security of blockciphers. Here, the key is presumed known, and the adversary succeeds in distinguishing if it identifies a structural property of the cipher. Andreeva et al. 11 proposed a way to formalize the known-key security of blockciphers based on the underlying primitives. The model is 
derived from the indifferentiability framework 37 and hence all composition results carry over. Intuitively: suppose some cryptosystem $\mathrm{F}$ is proven to achieve a certain level of security in the ideal permutation model, and consider $\mathrm{F}^{\prime}$ to be $\mathrm{F}$ with the permutations replaced by independent blockcipher instantiations. Then, $F^{\prime}$ achieves the same level of security as $F$, up to the known-key indifferentiability bound of the underlying blockciphers.

In [1, several blockcipher constructions are proven to be known-key indifferentiable, such as the multiple Even-Mansour cipher and 14 rounds of balanced Feistel with random functions (using a result of Holenstein et al. 24]). For such ciphers, the above approach works well, although for Even-Mansour the composition is trivial (one essentially replaces an ideal permutation by an ideal permutation) and for Feistel with 14 rounds security is only guaranteed up to $2^{n / 32}$ queries, where $n$ is the state size of the cipher.

Known-Key Attacks on Blockciphers. Knudsen and Rijmen also demonstrated that the Feistel network on $n$ bits with 7 rounds (called "Feistel 7 ") is not known-key indifferentiable 1.27): an adversary can generically find $2^{n / 2}$ plaintext/ciphertext tuples $(m, c)$ and $\left(m^{\prime}, c^{\prime}\right)$ satisfying $\mathrm{Ri}_{n / 2}\left(m \oplus c \oplus m^{\prime} \oplus c^{\prime}\right)=0$ (where $\mathrm{Ri}_{r}(x)$ outputs the $r$ rightmost bits of $x$ ). This result has lead to a wave of other known-key attacks on practical constructions, including generalized/extended variants of Feistel [1, 27, 47,53,56], reduced versions of AES or Rijndael 22, 27, 38, 44, 52, reduced variants of the blockciphers underlying SHA-2 and SHA-3 finalists BLAKE and Skein $2,2,7,31,34,61$, and many more [3, 11, 12, 14, 17, 18, 28, 33, 46, 47, 54, 55. This paper will mostly be concerned with differential known-key attacks, including reboundand boomerang-based attacks (the majority of above-mentioned attacks). We highlight two results that are among the best-known ones and that exemplify the idea of the other attacks. Gilbert and Peyrin 22 used the rebound technique 39 to derive a known-key attack on 8 rounds of AES (called "AES 8 "). It starts from the middle, and results in a differential trail with four active words in the beginning, and four at the end. These active words are overlapping at two positions, hence one could consider this result as two tuples $(m, c)$ and $\left(m^{\prime}, c^{\prime}\right)$ satisfying $m \oplus c \oplus m^{\prime} \oplus c^{\prime}=0$ at $10 n / 16$ bit-positions. The adversary has $2^{15} \leq 2^{n / 8}$ degrees of freedom in the attack, and for any choice it results in such a tuple with a certain probability. (The bound of $2^{n / 8}$ is used for simplicity later on.) The second attack we highlight is by $\mathrm{Yu}$ et al. 61, who employ the boomerang technique 60 to attack 36 rounds of the blockcipher Threefish-512 (called "Threefish 36 ") used in Skein. This attack results in four tuples $\left(m^{1}, c^{1}\right), \ldots,\left(m^{4}, c^{4}\right)$ satisfying $m^{1} \oplus \cdots \oplus c^{4}=0$. The adversary has $2^{n}$ degrees of freedom, but any trial succeeds with probability approximately $2^{-454}$. Therefore, the expected number of solutions is about $2^{n-454} \leq 2^{n / 8}$. This attack is in fact a knownrelated-key attack, where a fixed difference in the key exists. For simplicity, we condone this, observing that an attack with no key difference must logically be harder.

In any of these cases, the traditional and commonly employed ideal cipher/permutation model falls short: results achieved in this model do not necessarily hold if the primitives are instantiated with Feistel $_{7}, \mathrm{AES}_{8}$, Threefish 36 , or any other known-key distinguishable cipher.

\subsection{Our Contributions}

In their seminal work, Knudsen and Rijmen state: "In some cases blockciphers are used with a key that is known to the adversary, and at least to a certain extent, the key is under the adversary's control. Our attacks are quite relevant to this case." We investigate this fundamental question whether known-key attacks invalidate the security of primitive-based hash functions, but we do so in a much more general way. At a high level, we present a model that goes beyond the traditional ideal cipher model as well as the principle of known-key attacks and that allows to generically analyze the impact of various weaknesses of blockciphers on various blockcipher- and permutation-based cryptosystems.

Model. A naive approach to analyzing the impact of known-key attacks would be to simply plug a certain blockcipher construction into a hash function and to analyze its security, 
but this would be a devious and complex combinatorial task: for a function based on $r$ permutations, plugging Feistel ${ }_{7}$ into it would lead to $7 r$ underlying primitive calls. Note that proving security of the Feistel construction itself is already extraordinarily hard $16,24,32$. Instead, we model the blockciphers in such a way that they behave randomly, except that an adversary can exploit the particular relation. More formally, we pose a certain predicate $\Phi$, and we draw blockciphers randomly from the set of all ciphers that comply with predicate $\Phi$. Throughout, we refer to this model as the "weak cipher model (WCM)." It corresponds to the ideal cipher model if $\Phi$ is trivial.

We present an explicit description of a random weak cipher for the case where $\Phi$ implies for each key $k$ the existence of $A$ sets of $B$ queries $\left\{\left(k, m^{1}, c^{1}\right), \ldots,\left(k, m^{B}, c^{B}\right)\right\}$ that comply with a certain condition $\varphi$. These ciphers are modeled to have three interfaces: forward queries, inverse queries, and predicate queries. Forward and inverse queries are as usual; on a predicate query, an adversary is given a set of $B$ queries satisfying $\varphi$. Multiple technicalities are involved in this formalization. Most importantly, predicate $\Phi$ applies to tuples of queries, rather than single queries only, and some query responses may have a reduced entropy.

Above-mentioned known-key attacks are covered by our model if the condition $\varphi$ states for some $C \subseteq\{1, \ldots, n\}$ that

$$
\operatorname{Bits}_{C}\left(m^{1} \oplus c^{1} \oplus \cdots \oplus m^{B} \oplus c^{B}\right)=0,
$$

where $\operatorname{Bits}_{C}(x)$ outputs a string consisting of all bits of $x$ whose index is in $C$. (In fact, our model is much more general: above-mentioned attacks aim to generate only one relation, while we allow an adversary to see multiple relations.) The value $A$ usually depends on $n$ and $C$ is regularly a large subset. We consider $B$ being a relatively small number (independent of $n$ ). For the above-mentioned attack on Feistel ${ }_{7}, A=2^{n / 2}, B=2$, and $C$ corresponds to the rightmost $n / 2$ bits. Similarly, the attacks on $\mathrm{AES}_{8}$ (for $A=2^{n / 8}, B=2$, and $C$ a certain set of size 10n/16) and Threefish 36 (for $A=2^{n / 8}, B=4$, and $C=\{1, \ldots, n\}$ ) are covered, and so are almost all known differential (rebound- or boomerang-based) known-key attacks. We remark that, on the other hand, the predicate is not well-suited for integral-based known-key attacks: upon a predicate query an attacker would receive $B \approx 2^{n}$ queries.

The weak cipher model is similar to an approach followed by Bresson et al. 15] for the indifferentiability analysis of the SHA-3 candidate Shabal if the underlying blockcipher shows some non-random behavior, and by Bouillaguet et al. [13] to analyze the indifferentiability security of SIMD when the underlying compression function is distinguishable from a random function. However, in both approaches, the underlying biased primitives were relatively easy to model. For instance in 15 (using our terminology), predicate $\Phi$ is a relation that holds for single queries only, and not for combinations of queries. This considerably simplifies the analysis: one can derive a bias $\beta$ to measure the distance between primitive responses and fully random responses, and consider oracle responses to be drawn from a set of size at least $2^{n-\beta}$, and the original indifferentiability analysis carries over with minor modifications. The predicate used in the analysis in 13, on the other hand, does apply to tuples of queries, but the model can simply be described using two sampling algorithms, and an adversary cannot hit a weak pair by accident (which is possible in our analysis). Liskov [35 used a similar approach to prove indifferentiability security of the zipper hash if the underlying compression function is invertible up to a certain degree. However, the analysis is significantly simpler, as this primitive can be perfectly modeled. We finally remark that Katz et al. 26 analyze the impact of related-key attacks on blockciphers to hash functions. However, in their model, the differences $\Delta k, \Delta x, \Delta y$ are fixed, an ideal cipher is generated for half of the key space, and for the other half the cipher is adjusted as $\mathrm{E}_{k}(x, y)=\mathrm{E}_{k \oplus \Delta k}(x \oplus \Delta x) \oplus \Delta_{y}$. This primitive can be easily modeled, but is also too generous to the attacker.

To our knowledge, this is the first attempt to formally analyze the effect of a wide class of blockcipher attacks on higher level cryptographic functions. Nonetheless, the weak cipher model is in essence still a model: we use an abstraction of the cryptanalytic known-key attacks in such a way that the ideal cipher model can be relaxed to cope them. A further discussion on the accuracy of the model is given in Sect.77. 
Table 1. Security results for the PGV, Grøstl, and Shrimpton-Stam compression functions in the weak cipher model. Ideal cipher/permutation model bounds match the ones of $B \geq 3$. All results are tight except for the case $(B=1,|C|>n / 2)$ for Shrimpton-Stam.

\begin{tabular}{|c|c|c|c|c|c|c|c|}
\hline \multirow[b]{2}{*}{$B$} & \multirow[b]{2}{*}{$|C|$} & \multicolumn{2}{|c|}{ PGV } & \multicolumn{2}{|c|}{ Grøstl } & \multicolumn{2}{|c|}{ Shrimpton-Stam } \\
\hline & & collision & preimage & collision & preimage & collision & preimage \\
\hline \multirow[t]{2}{*}{1} & $\leq n / 2$ & $2^{(n-|C|) / 2}$ & $2^{n-|C|}$ & $2^{(n-|C|) / 4}$ & $2^{(n-|C|) / 2}$ & $2^{(n-|C|) / 2}$ & $2^{n / 2}$ \\
\hline & $>n / 2$ & $2^{(n-|C|) / 2}$ & $2^{n-|C|}$ & $2^{(n-|C|) / 4}$ & $2^{(n-|C|) / 2}$ & $2^{(n-|C|) / 2}$ & $2^{n-|C|}$ \\
\hline \multirow[t]{2}{*}{2} & $\leq n / 2$ & $2^{n / 2}$ & $2^{n}$ & $2^{n / 4}$ & $2^{n / 2}$ & $2^{n / 2}$ & $2^{n / 2}$ \\
\hline & $>n / 2$ & $2^{n-|C|}$ & $2^{n}$ & $2^{(n-|C|) / 2}$ & $2^{n / 2}$ & $2^{n-|C|}$ & $2^{n / 2}$ \\
\hline$\geq 3$ & arbitrary & $2^{n / 2}$ & $2^{n}$ & $2^{n / 4}$ & $2^{n / 2}$ & $2^{n / 2}$ & $2^{n / 2}$ \\
\hline
\end{tabular}

Application to Blockcipher-Based Hash Functions. Preneel, Govaerts, and Vandewalle (PGV) 48] classified the 64 most basic ways of constructing a $2 n$-to- $n$-bit compression function from a blockcipher with $n$-bit key and $n$-bit state, and claimed security of 12 of them. A formal security analysis of these functions in the ICM has been performed by Black et al. [9], and later by Duo and Li [19, Stam [59], and Black et al. 10]. In more detail, in the ICM these constructions achieve tight collision security up to about $2^{n / 2}$ queries and preimage security up to about $2^{n}$ queries. Baecher et al. [4 recently showed that the 12 secure PGV functions can be divided into two classes, in such a way that if a primitive makes one function secure it makes the entire class secure.

As first application of our model, we consider the PGV compression functions in the $\mathrm{WCM}$ and derive collision and preimage bounds for general $(A, B, C)$. A schematic summary of the results for various $B$ and $C$ is given in Table 1 (we remark that $A$ is merely a technical parameter that has no influence on the results). We also show that the bounds are optimal, by providing matching attacks. Some of these attacks are similar to methods used in $[27,53,56$ to detect (near-)collisions in certain PGV modes of operations using known-key attacks.

Application to Permutation-Based Hash Functions. We also apply the WCM to permutation-based compression functions. This is particularly interesting for two reasons: (i) it allows us to understand the impact of distinguishers on permutations that are used in hash functions, and (ii) a blockcipher with a fixed and known key is a permutation and can be used as such. In more detail, we consider the Grøstl compression function [21] and the permutation-based equivalent of the Shrimpton-Stam compression function [57 (see also Fig. 4. In the IPM, the former is proven to achieve collision security up to $2^{n / 4}$ queries, where $n$ is the state size, and preimage security up to $2^{n / 2}[20$. Rogaway and Steinberger $[51]$ showed via an automated analysis that the latter function is collision and preimage resistant up to $2^{n / 2}$ queries (asymptotically). This has been confirmed in the generalized work of Mennink and Preneel 41].

A summary of our findings for the Grøstl and Shrimpton-Stam compression functions in the WCM is given in Table 1. All results are tight, except for the case $(B=1,|C|>n / 2)$ for Shrimpton-Stam, for which we leave proving tightness as an open problem. We remark that the analysis for these schemes is much more demanding as multiple primitives are involved.

Impact. An application of our formalization to the PGV functions and various permutationbased functions shows that these achieve a comparable level of security in the ideal and weak cipher model for a spectrum of choices for $(A, B, C)$. This result particularly implies that most relevant rebound-based (including [12, $22,28,38,52,53,56]$ ) and boomerang-based (including [2,7, 31, 54, 61]) known-key attacks known to date do not invalidate the security of such functions, or only have a little effect. For instance, the above-discussed attack on Feistel $_{7}$ satisfies $B=2$ and $|C|=n / 2$ and it does not affect the security; similarly for Threefish $_{36}$ for which $B=4$. The attack on $\mathrm{AES}_{8}$ is covered for $B=2$ and $|C|=10 \mathrm{n} / 16$, 
which demonstrates a slight security degradation to $2^{6 n / 16}$ for the PGV functions, but this may in part be due to our over-generosity to the adversary. We remark that, even though we focused on collision and preimage resistance, the techniques can be generalized to other security notions, such as near-collisions. This may entail differences in the security results.

We stress that these results do not mean that the analyzed functions are secure when the underlying permutations are instantiated with, say, Feistel 7 or Threefish ${ }_{36}$ : it only means that existing known-key attacks, or more general weaknesses such as relation (1), alone are not sufficient to invalidate the collision and preimage security of the construction. Indeed, more sophisticated attacks which are not yet covered by our application of the WCM may still invalidate the security of certain modes [6]. It remains a challenging open research problem to generalize the findings to underlying primitives that have multiple or different weaknesses.

\subsection{Outline}

In Sect. 2 we formally present the "weak cipher model," and in Sect. 3 we show how it relates to known-key attacks. We apply the model to the PGV functions in Sect. 4, to the Grøstl compression function in Sect. 5, and to Shrimpton-Stam in Sect. 6. We conclude this work in Sect. 7.

\section{Weak Cipher Model}

If $X$ is a set, by $x \stackrel{\$}{\leftarrow} X$ we denote the uniformly random sampling of an element from $X$. By $X \leftarrow x$, we denote $X \leftarrow X \cup\{x\}$. For a bit string $x$, its bits are numbered $x=x_{|x|} \cdots x_{2} x_{1}$. If $C \subseteq\{1, \ldots,|x|\}$, the function $\operatorname{Bits}_{C}(x)$ outputs a string consisting of all bits of $x$ whose index is in $C$. Abusing notation, $\operatorname{Bits}_{\bar{C}}(x)$ always denotes the remaining bits (technically, $\bar{C}=\{1, \ldots,|x|\} \backslash C)$. For $0 \leq r \leq|x|$, we consider $\operatorname{Ri}_{r}(x)$ that outputs the $r$ rightmost bits of $x$. In other words, $\operatorname{Ri}_{r}(x)=\operatorname{Bits}_{\{1, \ldots, r\}}(x)$. For a function $f$, by $\operatorname{dom}(f)$ and $\operatorname{rng}(f)$ we denote its domain and range, respectively.

\subsection{Security Model}

For $\kappa \geq 0$ and $n \geq 1$, by $\mathrm{BC}(\kappa, n)$ we denote the set of all blockciphers with $\kappa$-bit key operating on $n$ bits. If $\kappa=0, \operatorname{BC}(n):=\mathrm{BC}(0, n)$ denotes the set of all $n$-bit permutations. If $\Phi$ is a predicate, by $\mathrm{BC}[\Phi](\kappa, n)$ we denote the subset of ciphers of $\mathrm{BC}(\kappa, n)$ that satisfy predicate $\Phi$. For $\pi \in \mathrm{BC}[\Phi](\kappa, n)$, the input-output tuples are denoted $(k, x, z)$, where $\pi(k, x)=\pi_{k}(x)=z$ and $\pi^{-1}(k, z)=\pi_{k}^{-1}(z)=x$. The key $k$ is omitted in case $\kappa=0$.

Let $\mathrm{F}:\{0,1\}^{s} \rightarrow\{0,1\}^{n}$ be a compressing function instantiated with $\ell \geq 1$ primitives from $\mathrm{BC}[\Phi](\kappa, n)$, for some predicate $\Phi$. Throughout, we consider security of $\mathrm{F}$ in an idealized model: we consider an adversary $\mathcal{A}$ that is a probabilistic algorithm with oracle access to a randomly sampled primitive $\pi=\left(\pi_{1}, \ldots, \pi_{\ell}\right) \stackrel{\$}{\leftarrow} \mathrm{BC}[\Phi](\kappa, n)^{\ell} . \mathcal{A}$ is information-theoretic and its complexity is only measured by the number of queries made to its oracles. The adversary can make forward and inverse queries to its oracles, and these queries are stored in a query history $\mathcal{Q}$.

A collision-finding adversary $\mathcal{A}$ for $\mathrm{F}$ aims at finding two distinct inputs to $\mathrm{F}$ that compress to the same range value. In more detail, we say that $\mathcal{A}$ succeeds if it finds two distinct inputs $X, X^{\prime}$ such that $\mathrm{F}(X)=\mathrm{F}\left(X^{\prime}\right)$ and $\mathcal{Q}$ contains all queries required for these evaluations of $F$. We define by

$$
\operatorname{Adv}_{\mathrm{F}}^{\mathrm{col}}(\mathcal{A})=\operatorname{Pr}\left(\boldsymbol{\pi} \stackrel{\$}{\leftarrow} \mathrm{BC}[\Phi](\kappa, n)^{\ell}, X, X^{\prime} \leftarrow \mathcal{A}^{\boldsymbol{\pi}}: X \neq X^{\prime} \wedge \mathrm{F}(X)=\mathrm{F}\left(X^{\prime}\right)\right)
$$

the probability that $\mathcal{A}$ succeeds in this. $\operatorname{By} \operatorname{Adv}_{\mathrm{F}}^{\text {col }}(q)$ we define the maximum collision advantage taken over all adversaries making $q$ queries. 
For preimage resistance, we focus on everywhere preimage resistance 50, which captures preimage security for every point of $\{0,1\}^{n}$. Let $Z \in\{0,1\}^{n}$ be any range value. Then, we say that $\mathcal{A}$ succeeds in finding a preimage if it obtains an input $X$ such that $\mathrm{F}(X)=Z$ and $\mathcal{Q}$ contains all queries required for this evaluation of $\mathrm{F}$. We define by

$$
\operatorname{Adv}_{\mathrm{F}}^{\mathrm{epre}}(\mathcal{A})=\max _{Z \in\{0,1\}^{n}} \operatorname{Pr}\left(\boldsymbol{\pi} \stackrel{\$}{\leftarrow} \mathrm{BC}[\Phi](\kappa, n)^{\ell}, X \leftarrow \mathcal{A}^{\boldsymbol{\pi}}(Z): \mathrm{F}(X)=Z\right)
$$

the probability that $\mathcal{A}$ succeeds, maximized over all possible choices for $Z$. By $\mathbf{A d v}_{\mathrm{F}}^{\text {epre }}(q)$ we define the maximum (everywhere) preimage advantage taken over all adversaries making $q$ queries.

If $\Phi$ is a trivial relation, we have $\mathrm{BC}[\Phi](\kappa, n)=\mathrm{BC}(\kappa, n)$, and the above definitions boil down to security in the ideal cipher model (ICM) if $\kappa>0$ or the ideal permutation model (IPM) if $\kappa=0$. On the other hand, if $\Phi$ is a non-trivial predicate, it strictly reduces the set $\mathrm{BC}(\kappa, n)$. In this case, we will refer to the model as the "weak cipher model (WCM)," for both $\kappa>0$ and $\kappa=0$. Very informally, this model still involves random ciphers/permutations, with the difference that an adversary may exploit a certain additional property. The modeling of a randomly drawn weak ciphers is much more delicate.

\subsection{Random Weak Cipher}

For a certain class of predicates, we discuss how to model a randomly drawn weak cipher $\pi$ from $\mathrm{BC}[\Phi](\kappa, n)$. Let $A, B \in \mathbb{N}$. We will consider predicates that imply, for every $k \in$ $\{0,1\}^{\kappa}$, the existence of $A$ sets of $B$ distinct queries $\left\{\left(x^{1}, z^{1}\right), \ldots,\left(x^{B}, z^{B}\right)\right\}$ that satisfy $\varphi_{k}\left(\left\{\left(x^{1}, z^{1}\right), \ldots,\left(x^{B}, z^{B}\right)\right\}\right)$ for some condition $\varphi$ depending on key $k$. The predicate is denoted $\Phi(A, B, \varphi)$. $A$ is merely a technical parameter, and throughout we assume it is larger than $q$, the number of oracle calls an adversary can make. This definition of $\Phi(A, B, \varphi)$ is fairly general. Particularly, predicate $B$-sets may overlap and the condition $\varphi$ can represent any function on the inputs. We note that $\Phi$ can be easily generalized to tuples of different length and/or to multiple types of conditions at the same time.

Traditionally, an adversary has only forward $\pi_{k}(x)$ and inverse $\pi_{k}^{-1}(z)$ query access. In order for the adversary to be able to exploit the weakness present in $\pi$, we give it additional access to $\pi$ via a "predicate query" $\pi_{k}^{\Phi}(y)$ : on input of $y \in\{1, \ldots, A\}$, the adversary obtains a $B$-set $\left\{\left(x^{1}, z^{1}\right), \ldots,\left(x^{B}, z^{B}\right)\right\}$ that satisfies $\varphi_{k}\left(\left\{\left(x^{1}, z^{1}\right), \ldots,\left(x^{B}, z^{B}\right)\right\}\right)$.

A formal description of how to model $\pi \stackrel{\$}{\leftarrow} \mathrm{BC}[\Phi(A, B, \varphi)](\kappa, n)$ is given in Fig. 1 Here, for every $k \in\{0,1\}^{\kappa}, P_{k}$ is an initially empty list of $\pi_{k}$-evaluations, where a regular forward/inverse query adds one element $(x, z)$ to $P_{k}$ and a $\pi_{k}^{\Phi}$-query may add up to $B$ elements. Additionally, $P_{k}^{\Phi}$ is an initially empty list of queries to $\pi_{k}^{\Phi}$. We denote by $\Sigma_{k}\left(P_{k}, P_{k}^{\Phi}\right) \subseteq\left(\{0,1\}^{n} \times\{0,1\}^{n}\right)^{B}$ the set of all tuples $\left\{\left(x^{1}, z^{1}\right), \ldots,\left(x^{B}, z^{B}\right)\right\}$ such that

(i) $x^{1}, \ldots, x^{B}$ are pairwise distinct and $z^{1}, \ldots, z^{B}$ are pairwise distinct;

(ii) $\forall_{\ell=1}^{B}: x^{\ell} \in \operatorname{dom}\left(P_{k}\right) \Longrightarrow z^{\ell}=P_{k}\left(x^{\ell}\right)$ and $z^{\ell} \in \operatorname{rng}\left(P_{k}\right) \Longrightarrow x^{\ell}=P_{k}^{-1}\left(z^{\ell}\right)$;

(iii) $\varphi_{k}\left(\left\{\left(x^{1}, z^{1}\right), \ldots,\left(x^{B}, z^{B}\right)\right\}\right)$ holds;

(iv) $\left\{\left(x^{p(1)}, z^{p(1)}\right), \ldots,\left(x^{p(B)}, z^{p(B)}\right)\right\} \notin \operatorname{rng}\left(P_{k}^{\Phi}\right)$ for any permutation $p$ on $\{1, \ldots, B\}$.

For a new query $\pi_{k}^{\Phi}(y)$, the response is then randomly drawn from $\Sigma_{k}\left(P_{k}, P_{k}^{\Phi}\right)$. Conditions (i-iii) are fairly self-evident; note particularly that an existing $(x, z) \in P_{k}$ may appear in multiple predicate queries. Condition (iv) assures that the drawing from $\Sigma_{k}\left(P_{k}, P_{k}^{\Phi}\right)$ is not just an old predicate query or a reordering thereof. The usage of this set $\Sigma_{k}\left(P_{k}, P_{k}^{\Phi}\right)$ allows for a uniform behavior of $\pi_{k}^{\Phi}$ for every $k$, and in general of $\pi \stackrel{\$}{\leftarrow} \operatorname{BC}[\Phi(A, B, \varphi)](\kappa, n)$, modulo the known existence of condition $\varphi$. This step is fundamental to our model and new compared with previous approaches of $13,15,35$. We remark that the model allows adversaries to make their queries at their own discretion, e.g., duplicate queries and regular queries after predicate queries are allowed. 

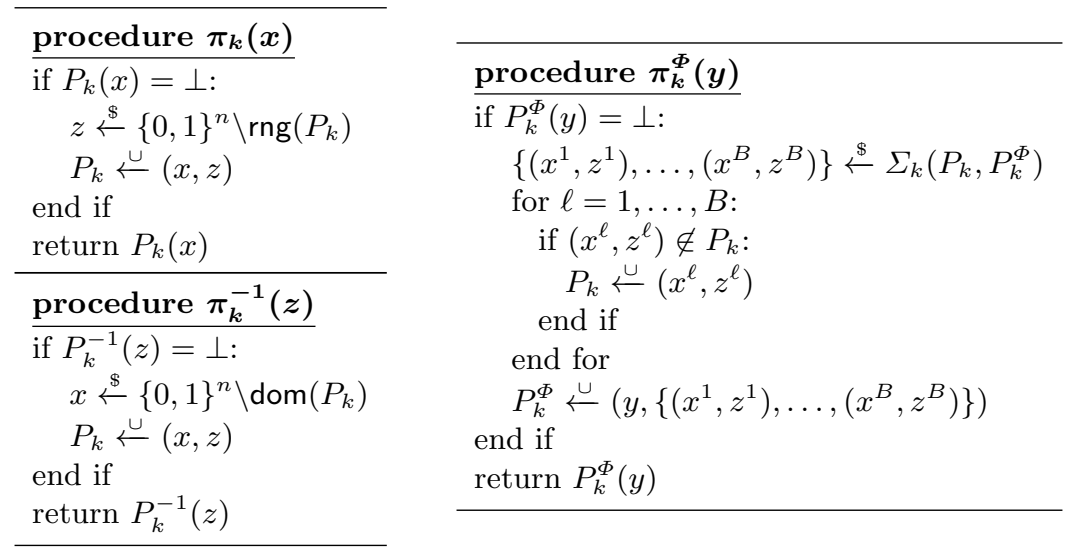

Fig. 1. Random weak cipher $\pi$. An adversary has access to $\pi, \pi^{-1}$, and $\pi^{\Phi}$.

\subsection{Random Abortable Weak Cipher}

Security analyses in the WCM are significantly more complex than in the ICM or IPM, which is in part because predicate queries may consist of older queries. This will particularly be an issue once collisions among queries are investigated. To suit the analysis for this case, we transform the WCM to an abortable weak cipher model (AWCM), which we denote as $\overline{\mathrm{BC}}[\Phi(A, B, \varphi)](\kappa, n)$. At a high-level, an abortable weak cipher responds to predicate queries with new query tuples only, and aborts once it turns out that an older query appears in a newer predicate query.

For any $k \in\{0,1\}^{\kappa}$ and partial $P_{k}$ and $P_{k}^{\Phi}$, define by $\bar{\Sigma}_{k}\left(P_{k}^{\Phi}\right) \subseteq\left(\{0,1\}^{n} \times\{0,1\}^{n}\right)^{B}$ the set of all tuples $\left\{\left(x^{1}, z^{1}\right), \ldots,\left(x^{B}, z^{B}\right)\right\}$ such that

(iii) $\varphi_{k}\left(\left\{\left(x^{1}, z^{1}\right), \ldots,\left(x^{B}, z^{B}\right)\right\}\right)$ holds;

(iv) $\left\{\left(x^{p(1)}, z^{p(1)}\right), \ldots,\left(x^{p(B)}, z^{p(B)}\right)\right\} \notin \operatorname{rng}\left(P_{k}^{\Phi}\right)$ for any permutation $p$ on $\{1, \ldots, B\}$.

$\bar{\Sigma}_{k}\left(P_{k}^{\Phi}\right)$ differs from $\Sigma\left(P_{k}, P_{k}^{\Phi}\right)$ in that conditions (i) and (ii) are omitted, and particularly: it is independent of $P_{k}$. A formal description of a random cipher $\bar{\pi} \stackrel{\$}{\leftarrow} \overline{\mathrm{BC}}[\Phi(A, B, \varphi)](\kappa, n)$ is given in Fig. 2. It deviates from Fig. 1 as follows: for every key $k, \bar{\pi}_{k}^{\Phi}$ responds randomly from $\bar{\Sigma}_{k}\left(P_{k}^{\Phi}\right)$, and it aborts if the response violates one of the two skipped conditions of $\Sigma_{k}\left(P_{k}, P_{k}^{\Phi}\right)$.

The next lemma shows that the WCM and AWCM are indistinguishable as long as the abortable weak cipher does not abort, approximately up to the birthday bound. Here, we assume that $\bar{\Sigma}_{k}\left(P_{k}^{\Phi}\right)$ is always large enough.

Lemma 1. Let $\bar{\pi} \stackrel{\$}{\leftarrow} \overline{\mathrm{BC}}\left[\Phi\left(A, B, \varphi^{C}\right)\right](\kappa, n)$. Consider an adversary that makes $q$ queries to $\bar{\pi}$. Then,

$$
\operatorname{Pr}(\bar{\pi} \text { sets abort }) \leq \frac{B^{2} q(q+1)}{2^{n}-\frac{B ! q 2^{n}}{\left|\bar{\Sigma}_{k}(\varnothing)\right|}} .
$$

Proof. Consider the $i^{\text {th }}$ query, for $i \in\{1, \ldots, q\}$, and assume it is a predicate query $\bar{\pi}_{k}^{\Phi}(y)$. We will consider the probability that this query makes $\bar{\pi}$ abort, provided it has not aborted so far. Prior to this $i^{\text {th }}$ query, $\left|P_{k}\right| \leq B(i-1)$ and $\left|P_{k}^{\Phi}\right| \leq i$. Basic combinatorics shows that

$$
\left|\bar{\Sigma}_{k}\left(P_{k}^{\Phi}\right)\right|=\left|\bar{\Sigma}_{k}(\varnothing)\right|-B ! \cdot\left|P_{k}^{\Phi}\right|,
$$

where we use that $\bar{\pi}$ has not aborted so far. This $i^{\text {th }}$ query aborts only if for some $\ell \in$ $\{1, \ldots, B\}$, the value $x^{\ell}$ equals an element in $\operatorname{dom}\left(P_{k}\right) \cup\left\{x^{1}, \ldots, x^{\ell-1}\right\}$ or the value $z^{\ell}$ equals an element in $\operatorname{rng}\left(P_{k}\right) \cup\left\{z^{1}, \ldots, z^{\ell-1}\right\}$. 

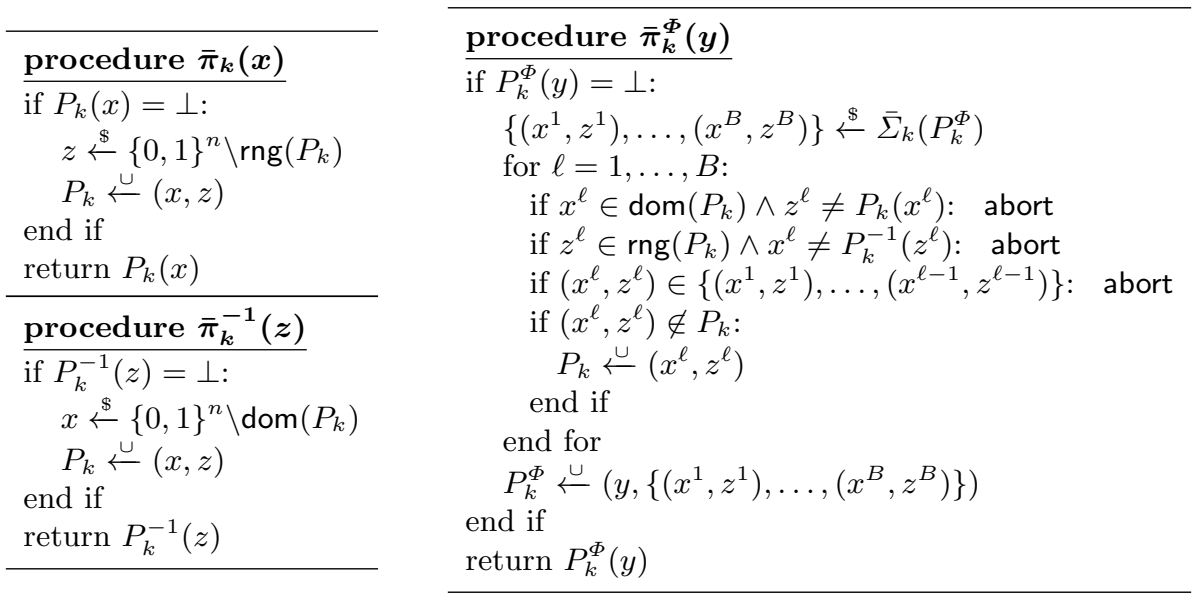

Fig. 2. Random abortable weak cipher $\bar{\pi}$. An adversary has access to $\bar{\pi}, \bar{\pi}^{-1}$, and $\bar{\pi}^{\Phi}$.

Define by $\bar{\Sigma}_{k}^{\text {abort }}\left(P_{k}^{\Phi}\right)$ the set of all elements of $\bar{\Sigma}_{k}\left(P_{k}^{\Phi}\right)$ that would lead to abort. We have $2 B$ possible values to cause the abort (namely, $x^{1}, \ldots, z^{B}$ ), and it causes the abort if it equals an element in a set of size at most $\left|P_{k}\right|+B$. For any of these $2 B\left(\left|P_{k}\right|+B\right)$ choices, the number of tuples in $\bar{\Sigma}_{k}\left(P_{k}^{\Phi}\right)$ complying with this choice is at most $\frac{\left|\bar{\Sigma}_{k}(\varnothing)\right|}{2^{n}}$. Thus,

$$
\operatorname{Pr}\left(\bar{\pi}^{\Phi}(y) \text { sets abort }\right)=\frac{\left|\bar{\Sigma}_{k}^{\text {abort }}\left(P_{k}^{\Phi}\right)\right|}{\left|\bar{\Sigma}_{k}\left(P_{k}^{\Phi}\right)\right|} \leq \frac{2 B\left(\left|P_{k}\right|+B\right) \cdot \frac{\left|\bar{\Sigma}_{k}(\varnothing)\right|}{2^{n}}}{\left|\bar{\Sigma}_{k}(\varnothing)\right|-B ! \cdot\left|P_{k}^{\Phi}\right|} \leq \frac{2 B^{2} i}{2^{n}-\frac{B ! q 2^{n}}{\left|\bar{\Sigma}_{k}(\varnothing)\right|}}
$$

The proof is completed by summation over $i=1, \ldots, q$.

\section{Modeling Known-Key Attacks}

We next apply the WCM to known-key attacks. For the sake of explanation, we first reconsider the Knudsen-Rijmen attack on Feistel $_{7}$ 27. (A detailed description of the attack is given in App. A.) Let $n \in \mathbb{N}$, and let $\pi:=\pi_{k}$ be an instance of Feistel 7 with fixed key $k$. Knudsen and Rijmen revealed four functions $f, f^{\prime}, g, g^{\prime}:\{0,1\}^{n / 2} \rightarrow\{0,1\}^{n}$ such that for all $y \in\{0,1\}^{n / 2}$ :

$$
\begin{aligned}
& g(y)=\pi(f(y)) \text { and } g^{\prime}(y)=\pi\left(f^{\prime}(y)\right), \\
& \operatorname{Ri}_{n / 2}(f(y) \oplus g(y))=\operatorname{Ri}_{n / 2}\left(f^{\prime}(y) \oplus g^{\prime}(y)\right) .
\end{aligned}
$$

These four functions correspond to the equations of (9) in App. A and depend on the cryptographic primitive underlying Feistel $_{7}$ in a complicated way. Therefore, we can safely assume that these functions behave sufficiently random, besides this particular relation (2), and that they are unknown to the adversary. $f, f^{\prime}, g, g^{\prime}$ are all injective and satisfy $f(y) \neq$ $f^{\prime}(y)$ and $g(y) \neq g^{\prime}(y)$ for all $y$. On the other hand, collisions of the form $f(y)=f^{\prime}\left(y^{\prime}\right)$ and $g(y)=g^{\prime}\left(y^{\prime}\right)$ may occur.

Generically, the attack demonstrates that for key $k$ there exist $2^{n / 2}$ possibly overlapping sets of distinct queries $\left\{\left(x^{1}, z^{1}\right),\left(x^{2}, z^{2}\right)\right\}$ that satisfy $\operatorname{Ri}_{n / 2}\left(x^{1} \oplus z^{1} \oplus x^{2} \oplus z^{2}\right)=0$. In other words, Feistel ${ }_{7}$ meets predicate $\Phi\left(2^{n / 2}, 2, \varphi^{\text {Feistel }_{7}}\right)$, where

$$
\varphi_{k}^{\text {Feistel }_{7}}\left(\left\{\left(x^{1}, z^{1}\right),\left(x^{2}, z^{2}\right)\right\}\right): \operatorname{Ri}_{n / 2}\left(x^{1} \oplus z^{1} \oplus x^{2} \oplus z^{2}\right)=0 .
$$

Here, we remark that the Knudsen-Rijmen attack works for any fixed but known key $k$, and that condition $\varphi_{k}^{\text {Feistel }_{7}}$ is in fact independent of the key. In this work, we will consider a 
more general predicate $\Phi\left(A, B, \varphi^{C}\right)$ for $A, B \in \mathbb{N}$ and $C \subseteq\{1, \ldots, n\}$, where

$$
\varphi_{k}^{C}\left(\left\{\left(x^{1}, z^{1}\right), \ldots,\left(x^{B}, z^{B}\right)\right\}\right): \operatorname{Bits}_{C}\left(x^{1} \oplus z^{1} \oplus \cdots \oplus x^{B} \oplus z^{B}\right)=0 .
$$

This generalized predicate considers the case of arbitrary but fixed and known keys, where the adversary can even choose the key every time it makes a predicate query. Note that also the attacks on $\mathrm{AES}_{8}$ and $\mathrm{Threefish}_{36}$ (see Sect. 1) are covered, as they satisfy $\Phi\left(2^{n / 8}, 2, \varphi^{C}\right.$ ) for certain $C$ of size $10 n / 16$ and $\Phi\left(2^{n / 8}, 4, \varphi^{\{1, \ldots, n\}}\right)$, respectively. In general, all rebound- or boomerang-based known-key attack in literature are covered by predicate $\Phi\left(A, B, \varphi^{C}\right)$ for some $A, B, C$. Here, $B$ is always a value independent of $n$ (usually 2 or 4 ) and $C$ is regularly a large subset (of size at least $n / 4$ ). Throughout, we consider $A$ to be sufficiently large.

\section{Basic Computations for AWCM}

For the specific condition $\varphi^{C}$ of $(3)$, we derive a simpler bound on the probability that a primitive $\bar{\pi} \stackrel{\$}{\leftarrow} \overline{\mathrm{BC}}\left[\Phi\left(A, B, \varphi^{C}\right)\right](\kappa, n)$ aborts, along with some other elementary observations for $\bar{\pi}$. To this end, we define the notation " $[X]$," which equals 1 if $X$ holds and 0 otherwise. For conciseness, we introduce the function $\delta_{B, C}[b]$ defined as

$$
\delta_{B, C}[b]=2^{|C|}[B=b]+[B>b]=\left\{\begin{array}{l}
2^{|C|} \text { if } B=b, \\
1 \text { if } B>b, \\
0 \text { otherwise }
\end{array}\right.
$$

Lemma 2. Let $\bar{\pi} \stackrel{\$}{\leftarrow} \overline{\mathrm{BC}}\left[\Phi\left(A, B, \varphi^{C}\right)\right](\kappa, n)$. Consider an adversary that makes $q \leq 2^{n-1} / B$ queries to $\bar{\pi}$. Then,

$$
\operatorname{Pr}(\bar{\pi} \text { sets abort }) \leq \frac{B^{2} q(q+1)}{2^{n}-B q} .
$$

Let $k \in\{0,1\}^{\kappa}$ and let $Z, Z^{\prime}, Z^{\prime \prime} \in\{0,1\}^{n}$. Consider any new query $\bar{\pi}_{k}^{\Phi}(y)$ and assume it does not abort. Write the response as $\left\{\left(x^{1}, z^{1}\right), \ldots,\left(x^{B}, z^{B}\right)\right\}$. Then,

(i) $\forall a \in\{1, \ldots, B\}: \operatorname{Pr}\left(x^{a}=Z\right), \operatorname{Pr}\left(z^{a}=Z\right) \leq \frac{1}{2^{n}-B q}$;

(ii) $\forall a \in\{1, \ldots, B\}: \operatorname{Pr}\left(x^{a} \oplus z^{a}=Z\right) \leq \frac{\delta_{B, C}[1]}{2^{n}-B q}$;

(iii) $\forall\{a, b\} \subseteq\{1, \ldots, B\}: \operatorname{Pr}\left(x^{a} \oplus z^{a}=Z \wedge x^{b} \oplus z^{b}=Z^{\prime}\right) \leq \frac{\delta_{B, C}[2]}{2^{2 n}-B q}$;

(iv) $\forall\{a, b\} \subseteq\{1, \ldots, B\}$ :

$$
\operatorname{Pr}\left(x^{a}=Z \wedge x^{b}=Z^{\prime} \wedge x^{a} \oplus z^{a} \oplus x^{b} \oplus z^{b}=Z^{\prime \prime}\right) \leq \frac{\delta_{B, C}[2]}{2^{3 n}-B q} .
$$

Proof. Recall from the proof of Lem. 1 that

$$
\left|\bar{\Sigma}_{k}\left(P_{k}^{\Phi}\right)\right|=\left|\bar{\Sigma}_{k}(\varnothing)\right|-B !\left|P_{k}^{\Phi}\right|,
$$

where $\left|P_{k}^{\Phi}\right| \leq q$. For the specific predicate analyzed in this lemma, $\left|\bar{\Sigma}_{k}(\varnothing)\right|=\left(2^{n}\right)^{2 B-1} 2^{n-|C|}$. In the remainder, we regularly bound $B ! \leq B \cdot\left(2^{n}\right)^{2 B-2}$ for $B \geq 1$ or $B ! \leq B \cdot\left(2^{n}\right)^{2 B-4}$ for $B \geq 2$.

Probability of abortion. The bound of (5) directly follows from Lem. 1, the abovementioned size of $\bar{\Sigma}_{k}(\varnothing)$, and the bound on $\bar{B}$ !.

Part (i). Define by $\bar{\Sigma}_{k}^{(\mathrm{i})}\left(P_{k}^{\Phi}\right)$ the set of all elements of $\bar{\Sigma}_{k}\left(P_{k}^{\Phi}\right)$ that satisfy $x^{a}=Z$. Then, $\left|\bar{\Sigma}_{k}^{(\mathrm{i})}\left(P_{k}^{\Phi}\right)\right| \leq\left(2^{n}\right)^{2 B-2} 2^{n-|C|}$, and

$$
\operatorname{Pr}\left(x^{a}=Z\right)=\frac{\left|\bar{\Sigma}_{k}^{(\mathrm{i})}\left(P_{k}^{\Phi}\right)\right|}{\left|\bar{\Sigma}_{k}\left(P_{k}^{\Phi}\right)\right|} \leq \frac{1}{2^{n}-B q} .
$$


A similar analysis applies to the case $z^{a}=Z$.

Part (ii). Define by $\bar{\Sigma}_{k}^{(\mathrm{ii})}\left(P_{k}^{\Phi}\right)$ the set of all elements of $\bar{\Sigma}_{k}\left(P_{k}^{\Phi}\right)$ that satisfy $x^{a} \oplus z^{a}=Z$. We make a distinction between $B=1$ and $B>1$. In case $B>1$, a similar reasoning as in (i) applies, and we have $\left|\bar{\Sigma}_{k}^{(\mathrm{ii})}\left(P_{k}^{\Phi}\right)\right| \leq\left(2^{n}\right)^{2 B-2} 2^{n-|C|}$. On the other hand, if $B=1$, we have $\left|\bar{\Sigma}_{k}^{(i i)}\left(P_{k}^{\Phi}\right)\right|=0$ if $\operatorname{Bits}_{C}(Z) \neq 0$ and $\left|\bar{\Sigma}_{k}^{(i i)}\left(P_{k}^{\Phi}\right)\right| \leq 2^{n}$ if $\operatorname{Bits}_{C}(Z)=0$. In any case,

$$
\left|\bar{\Sigma}_{k}^{(i i)}\left(P_{k}^{\Phi}\right)\right| \leq\left(2^{n}\right)^{2 B-2} 2^{n-|C|} \delta_{B, C}[1],
$$

and

$$
\operatorname{Pr}\left(x^{a} \oplus z^{a}=Z\right)=\frac{\left|\bar{\Sigma}_{k}^{(i i)}\left(P_{k}^{\Phi}\right)\right|}{\left|\bar{\Sigma}_{k}\left(P_{k}^{\Phi}\right)\right|} \leq \frac{\delta_{B, C}[1]}{2^{n}-B q} .
$$

Part (iii). This part only applies to $B>1$; if $B=1$ the probability equals 0 by construction. Define by $\bar{\Sigma}_{k}^{(\mathrm{iii})}\left(P_{k}^{\Phi}\right)$ the set of all elements of $\bar{\Sigma}_{k}\left(P_{k}^{\Phi}\right)$ that satisfy $x^{a} \oplus z^{a}=Z$ and $x^{b} \oplus z^{b}=$ $Z^{\prime}$. We make a distinction between $B=2$ and $B>2$. In case $B>2$, a similar reasoning as in (i) and (ii) applies, and we have $\left|\bar{\Sigma}_{k}^{(\text {iii) }}\left(P_{k}^{\Phi}\right)\right| \leq\left(2^{n}\right)^{2 B-3} 2^{n-|C|}$. On the other hand, if $B=2$, we have $\left|\bar{\Sigma}_{k}^{(\mathrm{iii})}\left(P_{k}^{\Phi}\right)\right|=0$ if $\operatorname{Bits}_{C}\left(Z \oplus Z^{\prime}\right) \neq 0$ and $\left|\bar{\Sigma}_{k}^{(\mathrm{iii})}\left(P_{k}^{\Phi}\right)\right| \leq\left(2^{n}\right)^{2}$ if $\operatorname{Bits}_{C}\left(Z \oplus Z^{\prime}\right)=0$. In any case,

$$
\left|\bar{\Sigma}_{k}^{(\mathrm{iii})}\left(P_{k}^{\Phi}\right)\right| \leq\left(2^{n}\right)^{2 B-3} 2^{n-|C|} \delta_{B, C}[2],
$$

and

$$
\operatorname{Pr}\left(x^{a} \oplus z^{a}=Z \wedge x^{b} \oplus z^{b}=Z^{\prime}\right)=\frac{\left|\bar{\Sigma}_{k}^{(i i i)}\left(P_{k}^{\Phi}\right)\right|}{\left|\bar{\Sigma}_{k}\left(P_{k}^{\Phi}\right)\right|} \leq \frac{\delta_{B, C}[2]}{2^{2 n}-B q} .
$$

Part (iv). The approach is fairly similar to case (iii). If $B=1$ the probability is 0 by construction. Define by $\bar{\Sigma}_{k}^{(\text {iv) }}\left(P_{k}^{\Phi}\right)$ the set of all elements of $\bar{\Sigma}_{k}\left(P_{k}^{\Phi}\right)$ that satisfy $x^{a}=Z$, $x^{b}=Z^{\prime}$, and $x^{a} \oplus z^{a} \oplus x^{b} \oplus z^{b}=Z^{\prime \prime}$. In case $B>2$, we have $\left|\bar{\Sigma}_{k}^{\text {(iv) }}\left(P_{k}^{\Phi}\right)\right| \leq\left(2^{n}\right)^{2 B-4} 2^{n-|C|}$. On the other hand, if $B=2$, we have $\left|\bar{\Sigma}_{k}^{(\text {iv })}\left(P_{k}^{\Phi}\right)\right|=0$ if $\operatorname{Bits}_{C}\left(Z^{\prime \prime}\right) \neq 0$ and $\left|\bar{\Sigma}_{k}^{(\text {iv })}\left(P_{k}^{\Phi}\right)\right| \leq 2^{n}$ if $\operatorname{Bits}_{C}\left(Z^{\prime \prime}\right)=0$. In any case,

$$
\left|\bar{\Sigma}_{k}^{(\mathrm{iv})}\left(P_{k}^{\Phi}\right)\right| \leq\left(2^{n}\right)^{2 B-4} 2^{n-|C|} \delta_{B, C}[2],
$$

and

$$
\operatorname{Pr}\left(x^{a}=Z \wedge x^{b}=Z^{\prime} \wedge x^{a} \oplus z^{a} \oplus x^{b} \oplus z^{b}=Z^{\prime \prime}\right)=\frac{\left|\bar{\Sigma}_{k}^{(\mathrm{iv})}\left(P_{k}^{\Phi}\right)\right|}{\left|\bar{\Sigma}_{k}\left(P_{k}^{\Phi}\right)\right|} \leq \frac{\delta_{B, C}[2]}{2^{3 n}-B q} .
$$

\section{Application to PGV Compression Functions}

We consider the 12 blockcipher-based compression functions from Preneel, Govaerts, and Vandewalle (PGV) 48. In the ICM these constructions achieve tight collision security up to about $2^{n / 2}$ queries and preimage security up to about $2^{n}$ queries $9,10,19,59$. The 12 constructions are depicted in Fig. 3. Here, we follow the ordering of [10], where PGV1, PGV2, and PGV5 are better known as the Matyas-Meyer-Oseas [36, Miyaguchi-Preneel, and Davies-Meyer [45] compression functions.

Baecher et al. 4] analyzed the 12 PGV constructions under ideal cipher reducibility, which at a high level covers the idea of two constructions being equally secure for the same underlying idealized blockcipher. They divide the PGV functions into two classes, in such a way that if some blockcipher makes one of the constructions secure, it makes all functions in the corresponding class secure. Applied to our WCM, the results of Baecher et al. imply the following: 

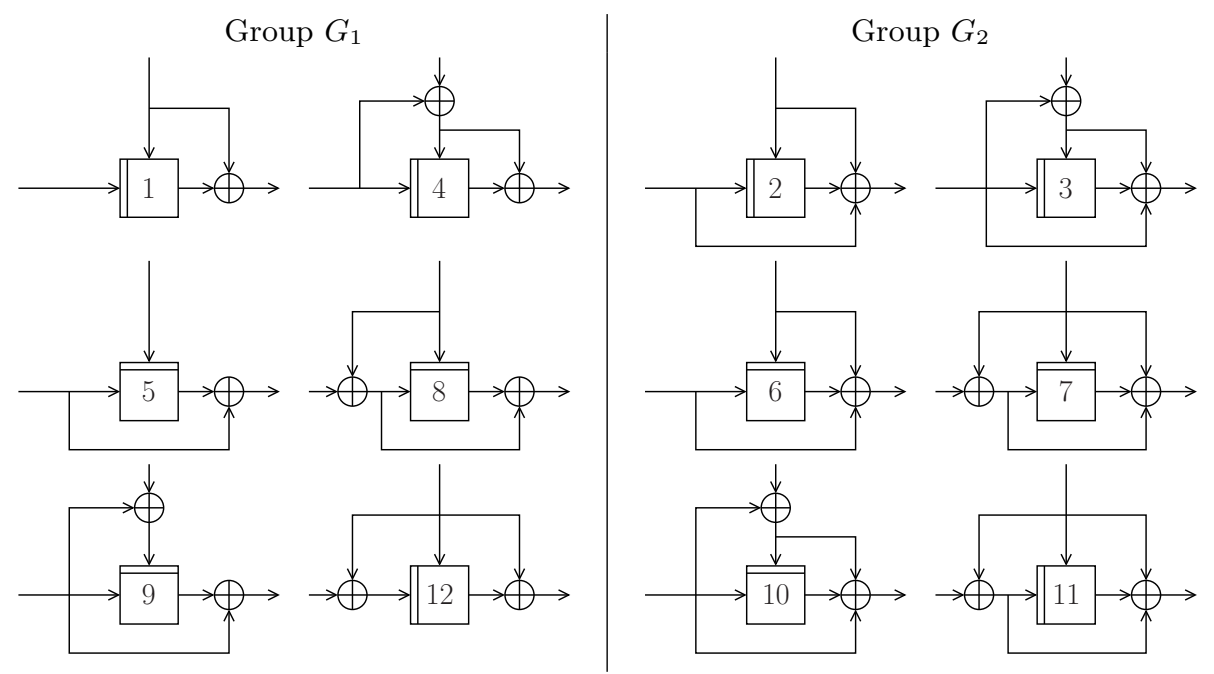

Fig. 3. The $12 \mathrm{PGV}$ compression functions. When in iteration mode, the message comes in at the top. The groups $G_{1}$ and $G_{2}$ refer to Lem. 3 .

Lemma 3 (Ideal Cipher Reducibility of PGV [4], informal). Let $\pi \stackrel{\&}{\leftarrow} \mathrm{BC}[\Phi](n, n)$ for some predicate $\Phi$. Let

$$
G_{1}=\{1,4,5,8,9,12\} \text {, and } G_{2}=\{2,3,6,7,10,11\} .
$$

For any $\alpha \in\{1,2\}$ and $i, j \in G_{\alpha}$, PGVi and PGV $j$ achieve the same level of collision and preimage security once instantiated with $\pi$.

Baecher et al. also derive a reduction between the two classes, but this reduction requires a non-direct transformation on the ideal cipher $\left.\pi\right|^{1}$ making it unsuitable for our purposes. Thanks to Lem. 3, it suffices to only analyze PGV1 and PGV2 in the WCM: the bounds carry over to the other 10 PGV constructions. In Sect. 4.1 we analyze the collision security of these functions in the WCM. The preimage security is considered in Sect. 4.2 .

\subsection{Collision Security}

Theorem 1. Let $n \in \mathbb{N}$. Let $\alpha \in\{1,2\}$ and consider PGV $\alpha$. Suppose $\pi \stackrel{\&}{\leftarrow}$ $\mathrm{BC}\left[\Phi\left(A, B, \varphi^{C}\right)\right](n, n)$. Then, for $q \leq 2^{n-1} / B$,

$$
\operatorname{Adv}_{\mathrm{PGV} \alpha}^{\mathrm{col}}(q) \leq \frac{B^{2} \delta_{B, C}[1] q^{2}}{2^{n}}+\left(\begin{array}{c}
B \\
2
\end{array}\right) \frac{2 \delta_{B, C}[2] q}{2^{n}}+\frac{4 B^{2} q^{2}}{2^{n}} .
$$

Proof. We focus on PGV2. The analysis for PGV1 is a simplification due to the absence of the feed-forward of the key. We consider any adversary that has query access to $\pi \stackrel{\&}{\leftarrow}$ $\mathrm{BC}\left[\Phi\left(A, B, \varphi^{C}\right)\right](n, n)$ and makes $q$ queries. As a first step, we move from $\pi$ to $\bar{\pi} \leftarrow$ $\overline{\mathrm{BC}}\left[\Phi\left(A, B, \varphi^{C}\right)\right](n, n)$. By Lem. 2 this costs us an additional term $\frac{B^{2} q(q+1)}{2^{n}-B q}$.

A collision for PGV2 would imply the existence of two distinct query pairs $(k, x, z)$, $\left(k^{\prime}, x^{\prime}, z^{\prime}\right)$ such that $k \oplus x \oplus z=k^{\prime} \oplus x^{\prime} \oplus z^{\prime}$. We consider the $i^{\text {th }}$ query $(i \in\{1, \ldots, q\})$ to be the first query to make this condition satisfied, and sum over $i=1, \ldots, q$ at the end. For regular (forward or inverse) queries, the analysis of $[9,10,59$ mostly carries over. The analysis of predicate queries is a bit more technical.

\footnotetext{
${ }^{1}$ If $\pi$ makes the PGV constructions from group $G_{1}$ secure, there is a transformation $\tau$ such that $\tau^{\pi}$ makes the constructions from $G_{2}$ secure, and vice versa.
} 
Query $\bar{\pi}_{k}(x)$ or $\bar{\pi}_{k}^{-1}(z)$. The cases are the same by symmetry, and we consider $\bar{\pi}_{k}(x)$ only. Denote the response by $z$. There are at most $B(i-1)$ possible $\left(k^{\prime}, x^{\prime}, z^{\prime}\right)$. As $z$ is randomly drawn from a set of size at least $2^{n}-B q$, it satisfies $z=k \oplus x \oplus k^{\prime} \oplus x^{\prime} \oplus z^{\prime}$ with probability at most $\frac{B(i-1)}{2^{n}-B q}$.

Query $\overline{\boldsymbol{\pi}}_{\boldsymbol{k}}^{\boldsymbol{\Phi}}(\boldsymbol{y})$. Denote the query response by $\left\{\left(k, x^{1}, z^{1}\right), \ldots,\left(k, x^{B}, z^{B}\right)\right\}$. In case the $B$ set contributes only to $(k, x, z)$, the same reasoning as for regular queries applies with the difference that any query of the $B$-set may be successful and that the bound of Lem. 2 part (ii) applies: $\frac{B^{2} \delta_{B, C}[1](i-1)}{2^{n}-B q}$.

Now, consider the case the predicate query contributes to both $(k, x, z)$ and $\left(k, x^{\prime}, z^{\prime}\right)$. There are $\left(\begin{array}{c}B \\ 2\end{array}\right)$ ways for the predicate query to contribute (or 0 if $B=1$ ). By Lem. 2 part (iii), which considers the success probability for any such combination, the predicate query results in a collision with probability at most $\left(\begin{array}{c}B \\ 2\end{array}\right) \frac{\delta_{B, C}[2] 2^{n}}{2^{2 n}-B q}$.

Conclusion. Taking the maximum of all success probabilities, the $i^{\text {th }}$ query is successful with probability at most $\frac{B^{2} \delta_{B, C}[1](i-1)}{2^{n}-B q}+\left(\begin{array}{c}B \\ 2\end{array}\right) \frac{\delta_{B, C}[2] 2^{n}}{2^{2 n}-B q}$. Summation over $i=1, \ldots, q$ gives

$$
\mathbf{A d v}_{\mathrm{PGV} 2}^{\mathrm{col}}(q) \leq \frac{B^{2} \delta_{B, C}[1] q^{2}}{2\left(2^{n}-B q\right)}+\left(\begin{array}{c}
B \\
2
\end{array}\right) \frac{\delta_{B, C}[2] q}{2^{n}-B q}+\frac{B^{2} q(q+1)}{2^{n}-B q},
$$

where the last part of the bound comes from the transition from WCM to AWCM. The proof is completed by using the fact that $2^{n}-B q \geq 2^{n-1}$ for $B q \leq 2^{n-1}$, and that $q+1 \leq 2 q$ for $q \geq 1$.

We note that the bound gets worse for increasing values of $B$. This has a technical cause: predicate queries are counted equally expensive as regular queries, but result in up to $B$ new query tuples. This leads to several factors of $B$ in the bound. As this work is mainly concerned with differential known-key attacks for which $B$ is regularly small, these factors are of no major influence.

The implications of the bound of Thm. 1 become more visible when considering particular choices of $B$ and $C$.

(i) If $B=1$, then $\operatorname{Adv}_{\mathrm{PGV} \alpha}^{\mathrm{col}}(q) \leq \frac{2^{|C|} q^{2}}{2^{n}}+\frac{4 q^{2}}{2^{n}}$;

(ii) If $B=2$, then $\operatorname{Adv}_{\mathrm{PGV} \alpha}^{\mathrm{col}}(q) \leq \frac{20 q^{2}}{2^{n}}+\frac{4 \cdot 2^{|C|} q}{2^{n}}$;

(iii) If $B \geq 3$ (independent of $n$ ), then $\operatorname{Adv}_{\mathrm{PGV} \alpha}^{\text {col }}(q) \leq \frac{5 B^{2} q^{2}}{2^{n}}+\frac{B^{2} q}{2^{n}}$.

In other words, for $B=2$ and $C$ with $|C| \leq n / 2$, or for $B \geq 3$ constant and $C$ arbitrary, the PGV functions achieve the same $2^{n / 2}$ collision security level as in the ICM. On the other hand, if $B=1$, collisions can be found in about $2^{(n-|C|) / 2}$ queries, and if $B=2$ with $|C|>n / 2$, in about $2^{n-|C|}<2^{n / 2}$ queries. See also Table 1.

\section{Tightness}

For the cases $B=1$ and $C$ arbitrary, and $B=2$ and $C$ arbitrary such that $|C|>n / 2$, we derive generic attacks that demonstrate tightness of the bound of Thm. 1. Knudsen and Rijmen [27] and Sasaki et al. [53,56] already considered how to exploit a known-key pair for the underlying blockcipher to find a collision for the Matyas-Meyer-Oseas (PGV1) and/or Miyaguchi-Preneel (PGV2) compression functions. Their attacks correspond to our $B=2$ case.

Proposition $1(\boldsymbol{B}=\mathbf{1})$. Let $n \in \mathbb{N}$. Let $\alpha \in\{1,2\}$ and consider PGV $\alpha$. Suppose $\pi \stackrel{\&}{\leftarrow}$ $\operatorname{BC}\left[\Phi\left(A, 1, \varphi^{C}\right)\right](n, n)$. Then, $\mathbf{A d v}_{\mathrm{PGV} \alpha}^{\mathrm{col}}(q) \geq \frac{q^{2}}{2^{n-|C|}}$.

Proof. We construct a collision-finding adversary $\mathcal{A}$ for PGV2. It fixes key $k=0$, and makes predicate queries to $\pi_{k}^{\Phi}$ on input of distinct values $y$ to obtain $q$ queries $\left(k, x_{y}, z_{y}\right)$ satisfying $\operatorname{Bits}_{C}\left(x_{y} \oplus z_{y}\right)=0$. Any two such queries collide on the entire state, $k \oplus x_{y} \oplus z_{y}=k \oplus x_{y^{\prime}} \oplus z_{y^{\prime}}$, with probability at least $\frac{q^{2}}{2^{n-|C|}}$. The attack for PGV1 is the same as we have taken $k=0$. 
Proposition $2(\boldsymbol{B}=\mathbf{2}$ and $|\boldsymbol{C}|>\boldsymbol{n} / \mathbf{2})$. Let $n \in \mathbb{N}$. Let $\alpha \in\{1,2\}$ and consider PGV $\alpha$. Suppose $\pi \stackrel{\&}{\leftarrow} \mathrm{BC}\left[\Phi\left(A, 2, \varphi^{C}\right)\right](n, n)$. Then, $\operatorname{Adv}_{\mathrm{PGV} \alpha}^{\mathrm{col}}(q) \geq \frac{q}{2^{n-|C|}}$.

Proof. We construct a collision-finding adversary $\mathcal{A}$ for PGV2. It fixes key $k=0$, and makes predicate queries to $\pi_{k}^{\Phi}$ on input of distinct values $y$ to obtain $q 2$-sets $\left\{\left(k, x_{y}^{1}, z_{y}^{1}\right),\left(k, x_{y}^{2}, z_{y}^{2}\right)\right\}$ satisfying $\operatorname{Bits}_{C}\left(x_{y}^{1} \oplus z_{y}^{1}\right)=\operatorname{Bits}_{C}\left(x_{y}^{2} \oplus z_{y}^{2}\right)$. These two queries collide on the entire state,

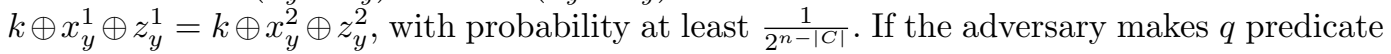
queries, we directly obtain our bound. The attack for PGV1 is the same as we have taken $k=0$.

\subsection{Preimage Security}

Theorem 2. Let $n \in \mathbb{N}$. Let $\alpha \in\{1,2\}$ and consider PGV $\alpha$. Suppose $\pi \stackrel{\$}{\leftarrow}$ $\mathrm{BC}\left[\Phi\left(A, B, \varphi^{C}\right)\right](n, n)$. Then, for $q \leq 2^{n-2} / B$,

$$
\operatorname{Adv}_{\mathrm{PGV} \alpha}^{\text {epre }}(q) \leq\left(\frac{2 B q}{2^{n}}\right)^{B}+\frac{2 B^{2} \delta_{B, C}[1] q}{2^{n}} .
$$

Due to space limitations, the proof is given in App. B. It is much more involved than the one of Thm. 1, particularly as we cannot make use of abortable ciphers. Entering various choices of $B$ and $C$ shows that in the PGV functions remain mostly unaffected in the WCM if $B \geq 2$, and the same security level as in the ICM is achieved [9, 10,59. A slight security degradation appears for $B=1$ as preimages can be found in about $2^{n-|C|}$.

\section{Tightness}

For the case $B=1$, we derive a generic attack that demonstrates the tightness of the bound of Thm. 2 ,

Proposition $3(\boldsymbol{B}=\mathbf{1})$. Let $n \in \mathbb{N}$. Let $\alpha \in\{1,2\}$ and consider PGV $\alpha$. Suppose $\pi \stackrel{\$}{\leftarrow}$ $\mathrm{BC}\left[\Phi\left(A, 1, \varphi^{C}\right)\right](n, n)$. Then, $\mathbf{A d v}_{\mathrm{PGV} \alpha}^{\mathrm{epre}}(q) \geq \frac{q}{2^{n-|C|}}$.

Proof. Let $Z$ be any given range value with $\operatorname{Bits}_{C}(Z)=0$ (note that epre guarantees security for every range point). A preimage-finding adversary $\mathcal{A}$ for PGV2 proceeds as follows. It fixes key $k=0$, and makes predicate queries to $\pi_{k}^{\Phi}$ on input of distinct values $y$ to obtain $q$ queries $\left(k, x_{y}, z_{y}\right)$ satisfying $\operatorname{Bits}_{C}\left(x_{y} \oplus z_{y}\right)=0$. Any such query hits $Z$ on the entire state,

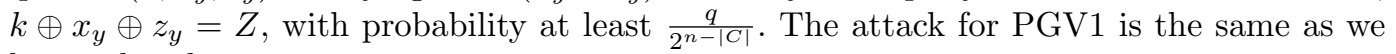
have taken $k=0$.

\section{Application to Grøstl Compression Function}

We consider the provable security of the compression function mode of operation of Grøstl 21] (see also Fig. 4):

$$
\mathrm{F}_{\mathrm{Gr} ø \mathrm{stl}}\left(x_{1}, x_{2}\right)=x_{2} \oplus \pi_{1}\left(x_{1}\right) \oplus \pi_{2}\left(x_{1} \oplus x_{2}\right) .
$$

The Grøstl compression function is in fact designed to operate in a wide-pipe mode, and in the IPM, the function is proven collision secure up to about $2^{n / 4}$ queries and preimage secure up to $2^{n / 2}$ queries 20. We consider the security of $\mathrm{F}_{\mathrm{Grøs}}$ in the WCM, where $\left(\pi_{1}, \pi_{2}\right) \stackrel{\&}{\leftarrow}$ $\mathrm{BC}\left[\Phi\left(A, B, \varphi^{C}\right)\right](n)^{2}$. We remark that in this section we consider keyless primitives, hence $\kappa=0$ and the $k$-input is dropped throughout. We furthermore note that finding collisions and preimages for $F_{\text {Grøstl }}$ is equivalent to finding them for

$$
\mathrm{F}_{\text {Grøstl }}^{\prime}\left(x_{1}, x_{2}\right)=x_{1} \oplus x_{2} \oplus \pi_{1}\left(x_{1}\right) \oplus \pi_{2}\left(x_{2}\right),
$$

as $\mathrm{F}_{\text {Grøstl }}\left(x_{1}, x_{2}\right)=\mathrm{F}_{\text {Grøstl }}^{\prime}\left(x_{1}, x_{1} \oplus x_{2}\right)$, and we will consider $\mathrm{F}_{\text {Grøstl }}^{\prime}$ throughout. 

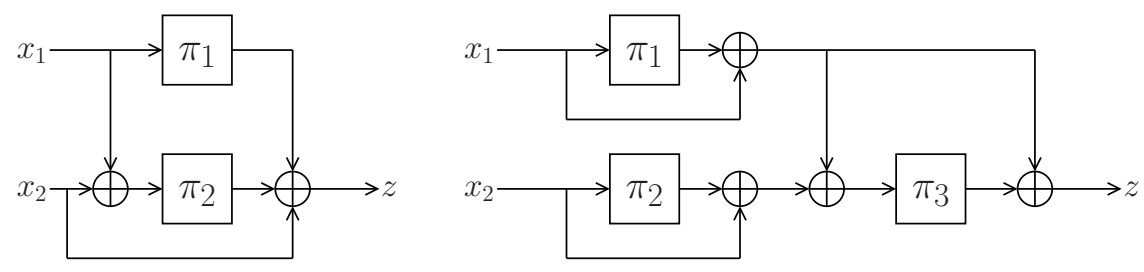

Fig. 4. Grøstl compression function (left) and Shrimpton-Stam (right).

\subsection{Collision Security}

Theorem 3. Let $n \in \mathbb{N}$. Suppose $\left(\pi_{1}, \pi_{2}\right) \stackrel{\&}{\leftarrow} \mathrm{BC}\left[\Phi\left(A, B, \varphi^{C}\right)\right](n)^{2}$. Then, for $q \leq 2^{n-1} / B$,

$$
\mathbf{A d v}_{\mathrm{F}_{\text {Grøstl }}^{\prime}}^{\mathrm{col}}(q) \leq \frac{B^{4} \delta_{B, C}[1] q^{4}}{2^{n}}+\left(\begin{array}{c}
B \\
2
\end{array}\right) \frac{2 \delta_{B, C}[2]\left(q^{2}+2^{n / 2-|C|} q\right)}{2^{n}}+\frac{B^{2} q^{2}}{2 \cdot 2^{n / 2}}+\frac{4 B^{2} q^{2}}{2^{n}}
$$

The proof is given in App. C. If we enter particular choices of $B$ and $C$ into the bound, we find results comparable to the case of Sect. 4.1. In more detail, for $B=2$ and $C$ with $|C| \leq n / 2$, or for $B \geq 3$ constant and $C$ arbitrary, $\mathrm{F}_{\mathrm{Gr} ø s t l}$ achieves the same $2^{n / 4}$ collision security level as in the ICM 20]. If $B=1$, the bound guarantees security up to about $2^{(n-|C|) / 4}$, and if $B=2$ with $|C|>n / 2$, collisions can be found in about $2^{(n-|C|) / 2}$ queries. See also Table 1. In App. D we show that the bound is optimal, by presenting tight attacks on $\mathrm{F}_{\text {Grøstl }}^{\prime}$ in the WCM.

\subsection{Preimage Security}

Theorem 4. Let $n \in \mathbb{N}$. Suppose $\left(\pi_{1}, \pi_{2}\right) \stackrel{\&}{\leftarrow} \mathrm{BC}\left[\Phi\left(A, B, \varphi^{C}\right)\right](n)^{2}$. Then, for $q \leq 2^{n-1} / B$,

$$
\mathbf{A d v}_{\mathbf{F}_{\text {Grostl }}^{\prime}}^{\text {epre }}(q) \leq \frac{2 B^{2} \delta_{B, C}[1]\left(q^{2}+2^{n / 2-|C|} q\right)}{2^{n}}+\frac{B q}{2^{n / 2}}+\frac{4 B^{2} q^{2}}{2^{n}}
$$

The proof is given in App. E As before, we find that $\mathrm{F}_{\mathrm{Grostl}}$ remains unaffected in the WCM for most cases, the sole exception being $B=1$ for which preimages can be found in about $2^{(n-|C|) / 2}$. In App. F we show that the bound is optimal, by presenting a tight attack on $\mathrm{F}_{\text {Grøstl }}^{\prime}$ for $B=1$ in the WCM.

\section{Application to Shrimpton-Stam Compression Function}

In this section, we consider the provable security of the Shrimpton-Stam compression function [57] (see also Fig. 4):

$$
\mathrm{F}_{\mathrm{SS}}\left(x_{1}, x_{2}\right)=x_{1} \oplus \pi_{1}\left(x_{1}\right) \oplus \pi_{3}\left(x_{1} \oplus \pi_{1}\left(x_{1}\right) \oplus x_{2} \oplus \pi_{2}\left(x_{2}\right)\right) .
$$

This function is proven asymptotically optimally collision and preimage secure up to $2^{n / 2}$ queries in the IPM $441,51,57]$. We consider the security of $\mathrm{F}_{\mathrm{SS}}$ in the WCM, where $\left(\pi_{1}, \pi_{2}, \pi_{3}\right) \stackrel{\$}{\leftarrow} \mathrm{BC}\left[\Phi\left(A, B, \varphi^{C}\right)\right](n)^{3}$. (As in Sect. 5 we consider keyless functions, hence $\kappa=0$ and the key inputs are dropped throughout.) Our findings readily apply to the generalization of $F_{S S}$ of 41]. The analysis of this construction is significantly more complex than

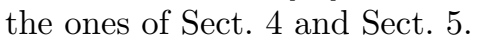




\subsection{Collision Security}

Theorem 5. Let $n \in \mathbb{N}$. Suppose $\left(\pi_{1}, \pi_{2}, \pi_{3}\right) \stackrel{\$}{\leftarrow} \mathrm{BC}\left[\Phi\left(A, B, \varphi^{C}\right)\right](n)^{3}$. Then,

(i) If $B=1$ and $C$ arbitrary, $\mathbf{A d v}_{\mathrm{F}_{\mathrm{SS}}}^{\text {col }}\left(2^{(n-|C|) / 2-n \varepsilon}\right) \rightarrow 0$ for $n \rightarrow \infty$;

(ii) If $B=2$ and $C$ with $|C| \leq n / 2, \mathbf{A d v}_{\mathrm{F}_{\mathrm{SS}}}^{\mathrm{col}}\left(2^{n / 2-n \varepsilon}\right) \rightarrow 0$ for $n \rightarrow \infty$;

(iii) If $B=2$ and $C$ with $|C|>n / 2, \mathbf{A d v}_{\mathbf{F}_{\mathrm{SS}}^{\text {col }}}^{\text {col }}\left(2^{n-|C|-n \varepsilon}\right) \rightarrow 0$ for $n \rightarrow \infty$;

(iv) If $B \geq 3$ (independent of $n$ ) and $C$ arbitrary, $\mathbf{A d v}_{\mathrm{F}_{\mathrm{SS}}}^{\mathrm{col}}\left(2^{n / 2-n \varepsilon}\right) \rightarrow 0$ for $n \rightarrow \infty$.

Due to the technicality of the proof, the results are expressed in asymptotic terms. The proof is given in App. G. For $B=2$ and $C$ with $|C| \leq n / 2$, or for $B \geq 3$ constant and $C$ arbitrary, $\mathrm{F}_{\mathrm{SS}}$ achieves the same security level as in the IPM. On the other hand, if $B=1$, or if $B=2$ but $|C|>n / 2$, Thm. 5 results in a worse bound. See also Table1. In App. H we show that the bound is optimal, by presenting tight attacks on $\mathrm{F}_{\mathrm{SS}}$ in the WCM.

\subsection{Preimage Security}

Theorem 6. Let $n \in \mathbb{N}$. Suppose $\left(\pi_{1}, \pi_{2}, \pi_{3}\right) \stackrel{\$}{\leftarrow} \mathrm{BC}\left[\Phi\left(A, B, \varphi^{C}\right)\right](n)^{3}$. Then,

(i) If $B=1$ and $C$ with $|C| \leq n / 2, \mathbf{A d v}_{\mathrm{F}_{\mathrm{SS}}}^{\text {epre }}\left(2^{n / 2-n \varepsilon}\right) \rightarrow 0$ for $n \rightarrow \infty$;

(ii) If $B=1$ and $C$ with $|C|>n / 2, \mathbf{A d v}_{\mathrm{F}_{\mathrm{SS}}}^{\text {epre }}\left(2^{n-|C|-n \varepsilon}\right) \rightarrow 0$ for $n \rightarrow \infty$;

(iii) If $B \geq 2$ (independent of $n$ ) and $C$ arbitrary, $\mathbf{A d v}_{\mathrm{F}_{\mathrm{SS}}}^{\mathrm{epre}}\left(2^{n / 2-n \varepsilon}\right) \rightarrow 0$ for $n \rightarrow \infty$.

As for collision resistance, the results are expressed in asymptotic terms. The proof is given in App. I. The bounds match the ones in the IPM, except for the case of $B=1$ and $|C|>n / 2$. We leave it as an open problem to prove tightness of Thm. 6 part (ii).

\section{Conclusions}

Since their formal introduction by Knudsen and Rijmen at ASIACRYPT 2007 [27], numerous known-key attacks on blockciphers have appeared in literature. These attacks are often considered delicate, as it is not always clear to what extent they influence the security of cryptographic functions based on these known-key blockciphers. We presented the weak cipher model in order to investigate this impact. For a specific instance of this model, considering the existence of $A$ sets of $B$ queries that satisfy condition $\varphi^{C}$ of (3), we proved that the PGV compression functions [48], the Grøstl compression function [21], and the Shrimpton-Stam compression function [57 remain mostly unaffected by the generalized weakness. Additionally, preimage security of the functions turned out to be significantly less susceptible to these types of weaknesses than collision security. The results can be readily generalized to other primitive-based functions, such as the double block length compression functions Tandem-DM, Abreast-DM, and Hirose's compression functions [23, 30], and to the permutation-based sponge mode 5 .

Our model is general enough to cover practically all differential known-key attacks in literature, such as latest results based on the rebound attack $12,22,28,38,52,53,56$ and on the boomerang attack $2,7,31,54,61$. To our knowledge, our work provides the first attempt to formally analyze the effect of a wide class of cryptanalytic attacks from a modular and provable security point of view. It is a step in the direction of security beyond the ideal model, connecting practical attacks from cryptanalysis with ideal model provable security. There is still a long way to go: in order to make the connection between the two fields, we abstracted known-key attacks to a certain degree. It remains a highly challenging open research problem to generalize our findings to multiple or different weaknesses, and to different permutationbased cryptographic functions. These generalizations include the analysis of known-key based constructions for more advanced conditions $\varphi$ (such as arbitrary polynomials). 
ACKNOWLEDGments. This work was supported in part by European Union's Horizon 2020 research and innovation programme under grant agreement No 644052 HECTOR and grant agreement No H2020-MSCA-ITN-2014-643161 ECRYPT-NET, and in part by the Research Council KU Leuven: GOA TENSE (GOA/11/007). Bart Mennink is a Postdoctoral Fellows of the Research Foundation - Flanders (FWO). The authors would like to thank the anonymous reviewers for their valuable help and feedback.

\section{References}

1. Andreeva, E., Bogdanov, A., Mennink, B.: Towards understanding the known-key security of block ciphers. In: Fast Software Encryption 2013. LNCS, vol. 8424, pp. 348-366. Springer, Heidelberg (2013)

2. Aumasson, J., Çalik, Çagdas., Meier, W., Özen, O., Phan, R., Varıcı, K.: Improved cryptanalysis of Skein. In: Advances in Cryptology - ASIACRYPT 2009. LNCS, vol. 5912, pp. 542-559. Springer, Heidelberg (2009)

3. Aumasson, J., Meier, W.: Zero-sum distinguishers for reduced Keccak- $f$ and for the core functions of Luffa and Hamsi (2009)

4. Baecher, P., Farshim, P., Fischlin, M., Stam, M.: Ideal-cipher (ir)reducibility for blockcipherbased hash functions. In: Advances in Cryptology - EUROCRYPT 2013. LNCS, vol. 7881, pp. 426-443. Springer, Heidelberg (2013)

5. Bertoni, G., Daemen, J., Peeters, M., Van Assche, G.: Sponge functions. ECRYPT Hash Function Workshop (2007)

6. Biryukov, A., Khovratovich, D., Nikolić, I.: Distinguisher and related-key attack on the full AES-256. In: Advances in Cryptology - CRYPTO 2009. LNCS, vol. 5677, pp. 231-249. Springer, Heidelberg (2009)

7. Biryukov, A., Nikolić, I., Roy, A.: Boomerang attacks on BLAKE-32. In: Fast Software Encryption 2011. LNCS, vol. 6733, pp. 218-237. Springer, Heidelberg (2011)

8. Black, J., Cochran, M., Shrimpton, T.: On the impossibility of highly-efficient blockcipherbased hash functions. In: Advances in Cryptology - EUROCRYPT 2005. LNCS, vol. 3494, pp. 526-541. Springer, Heidelberg (2005)

9. Black, J., Rogaway, P., Shrimpton, T.: Black-box analysis of the block-cipher-based hashfunction constructions from PGV. In: Advances in Cryptology - CRYPTO 2002. LNCS, vol. 2442, pp. 320-335. Springer, Heidelberg (2002)

10. Black, J., Rogaway, P., Shrimpton, T., Stam, M.: An analysis of the blockcipher-based hash functions from PGV. Journal of Cryptology 23(4), 519-545 (2010)

11. Blondeau, C., Peyrin, T., Wang, L.: Known-key distinguisher on full PRESENT. In: Advances in Cryptology - CRYPTO 2015, Part I. LNCS, vol. 9215, pp. 455-474. Springer, Heidelberg (2015)

12. Bouillaguet, C., Dunkelman, O., Leurent, G., Fouque, P.: Attacks on hash functions based on generalized feistel: Application to reduced-round Lesamnta and SHAvite-3512. In: Selected Areas in Cryptography 2010. LNCS, vol. 6544, pp. 18-35. Springer, Heidelberg (2010)

13. Bouillaguet, C., Fouque, P., Leurent, G.: Security analysis of SIMD. In: Selected Areas in Cryptography 2010. LNCS, vol. 6544, pp. 351-368. Springer, Heidelberg (2011)

14. Boura, C., Canteaut, A.: Zero-sum distinguishers for iterated permutations and application to Keccak- $f$ and Hamsi-256. In: Selected Areas in Cryptography 2010. LNCS, vol. 6544, pp. 1-17. Springer, Heidelberg (2010)

15. Bresson, E., Canteaut, A., Chevallier-Mames, B., Clavier, C., Fuhr, T., Gouget, A., Icart, T., Misarsky, J.F., Naya-Plasencia, M., Paillier, P., Pornin, T., Reinhard, J., Thuillet, C., Videau, M.: Indifferentiability with distinguishers: Why Shabal does not require ideal ciphers. Cryptology ePrint Archive, Report 2009/199 (2009)

16. Coron, J., Patarin, J., Seurin, Y.: The random oracle model and the ideal cipher model are equivalent. In: Advances in Cryptology - CRYPTO 2008. LNCS, vol. 5157, pp. 1-20. Springer, Heidelberg (2008)

17. Dong, L., Wu, W., Wu, S., Zou, J.: Known-key distinguisher on round-reduced 3D block cipher. In: Information Security Applications - WISA 2011. LNCS, vol. 7115, pp. 55-69. Springer, Heidelberg (2012)

18. Duan, M., Lai, X.: Improved zero-sum distinguisher for full round Keccak- $f$ permutation. Chinese Science Bulletin 57(6), 694-697 (2012) 
19. Duo, L., Li, C.: Improved collision and preimage resistance bounds on PGV schemes. Cryptology ePrint Archive, Report 2006/462 (2006)

20. Fouque, P., Stern, J., Zimmer, S.: Cryptanalysis of tweaked versions of SMASH and reparation. In: Selected Areas in Cryptography 2008. LNCS, vol. 5381, pp. 136-150. Springer, Heidelberg (2009)

21. Gauravaram, P., Knudsen, L.R., Matusiewicz, K., Mendel, F., Rechberger, C., Schläffer, M., Thomsen, S.: Grøstl - a SHA-3 candidate (2011), submission to NIST's SHA-3 competition

22. Gilbert, H., Peyrin, T.: Super-Sbox cryptanalysis: Improved attacks for AES-like permutations. In: Fast Software Encryption 2010. LNCS, vol. 6147, pp. 365-383. Springer, Heidelberg (2010)

23. Hirose, S.: Some plausible constructions of double-block-length hash functions. In: Fast Software Encryption 2006. LNCS, vol. 4047, pp. 210-225. Springer, Heidelberg (2006)

24. Holenstein, T., Künzler, R., Tessaro, S.: The equivalence of the random oracle model and the ideal cipher model, revisited. In: Proc. ACM Symposium on Theory of Computing 2011. pp. 89-98. ACM, New York (2011)

25. Jetchev, D., Özen, O., Stam, M.: Collisions are not incidental: A compression function exploiting discrete geometry. In: Theory of Cryptography Conference 2012. LNCS, vol. 7194, pp. 303-320. Springer, Heidelberg (2012)

26. Katz, J., Lucks, S., Thiruvengadam, A.: Hash functions from defective ideal ciphers. In: CTRSA 2015. LNCS, vol. 9048, pp. 273-290. Springer, Heidelberg (2015)

27. Knudsen, L., Rijmen, V.: Known-key distinguishers for some block ciphers. In: Advances in Cryptology - ASIACRYPT 2007. LNCS, vol. 4833, pp. 315-324. Springer, Heidelberg (2007)

28. Koyama, T., Sasaki, Y., Kunihiro, N.: Multi-differential cryptanalysis on reduced DMPRESENT-80: collisions and other differential properties. In: Information Security and Cryptology - ICISC 2012. Lecture Notes in Computer Science, vol. 7839, pp. 352-367. Springer, Heidelberg (2013)

29. Kuwakado, H., Hirose, S.: Hashing mode using a lightweight blockcipher. In: IMA International Conference 2013. LNCS, vol. 8308, pp. 213-231. Springer, Heidelberg (2013)

30. Lai, X., Massey, J.: Hash function based on block ciphers. In: Advances in Cryptology - EUROCRYPT '92. LNCS, vol. 658, pp. 55-70. Springer, Heidelberg (1992)

31. Lamberger, M., Mendel, F.: Higher-order differential attack on reduced SHA-256. Cryptology ePrint Archive, Report 2011/037 (2011)

32. Lampe, R., Seurin, Y.: Security analysis of key-alternating Feistel ciphers. In: Fast Software Encryption 2014. LNCS, vol. 8540, pp. 243-264. Springer, Heidelberg (2015)

33. Lauridsen, M.M., Rechberger, C.: Linear distinguishers in the key-less setting: Application to PRESENT. In: Fast Software Encryption 2015. LNCS, vol. 9054, pp. 217-240. Springer, Heidelberg (2015)

34. Leurent, G., Roy, A.: Boomerang attacks on hash function using auxiliary differentials. In: CT-RSA 2012. LNCS, vol. 7178, pp. 215-230. Springer, Heidelberg (2012)

35. Liskov, M.: Constructing an ideal hash function from weak ideal compression functions. In: Selected Areas in Cryptography 2006. LNCS, vol. 4356, pp. 358-375. Springer, Heidelberg (2007)

36. Matyas, S., Meyer, C., Oseas, J.: Generating strong one-way functions with cryptographic algorithm. IBM Techn. Disclosure Bull. 27(10A), 5658-5659 (1985)

37. Maurer, U., Renner, R., Holenstein, C.: Indifferentiability, impossibility results on reductions, and applications to the random oracle methodology. In: Theory of Cryptography Conference 2004. LNCS, vol. 2951, pp. 21-39. Springer, Heidelberg (2004)

38. Mendel, F., Peyrin, T., Rechberger, C., Schläffer, M.: Improved cryptanalysis of the reduced Grøstl compression function, ECHO permutation and AES block cipher. In: Selected Areas in Cryptography 2009. LNCS, vol. 5867, pp. 16-35. Springer, Heidelberg (2009)

39. Mendel, F., Rechberger, C., Schläffer, M., Thomsen, S.S.: The rebound attack: Cryptanalysis of reduced Whirlpool and Grøstl. In: Fast Software Encryption 2009. LNCS, vol. 5665, pp. 260-276. Springer, Heidelberg (2009)

40. Mennink, B.: Optimal collision security in double block length hashing with single length key. In: Advances in Cryptology - ASIACRYPT 2012. LNCS, vol. 7658, pp. 526-543. Springer, Heidelberg (2012)

41. Mennink, B., Preneel, B.: Hash functions based on three permutations: A generic security analysis. In: Advances in Cryptology - CRYPTO 2012. LNCS, vol. 7417, pp. 330-347. Springer, Heidelberg (2012) 
42. Mennink, B., Preneel, B.: Efficient parallelizable hashing using small non-compressing primitives. International Journal of Information Security (2015), to appear

43. Meyer, C., Schilling, M.: Secure program load with manipulation detection code. In: Proc. Securicom. pp. 111-130 (1988)

44. Minier, M., Phan, R., Pousse, B.: Distinguishers for ciphers and known key attack against Rijndael with large blocks. In: Progress in Cryptology - AFRICACRYPT 2009. LNCS, vol. 5580, pp. 60-76. Springer, Heidelberg (2009)

45. Miyaguchi, S., Ohta, K., Iwata, M.: Confirmation that some hash functions are not collision free. In: Advances in Cryptology - EUROCRYPT '90. LNCS, vol. 473, pp. 326-343. Springer, Heidelberg (1990)

46. Nakahara Jr., J.: New impossible differential and known-key distinguishers for the 3D cipher. In: Information Security Practice and Experience - ISPEC 2011. LNCS, vol. 6672, pp. 208-221. Springer, Heidelberg (2011)

47. Nikolić, I., Pieprzyk, J., Sokolowski, P., Steinfeld, R.: Known and chosen key differential distinguishers for block ciphers. In: Information Security and Cryptology - ICISC 2010. LNCS, vol. 6829, pp. 29-48. Springer, Heidelberg (2010)

48. Preneel, B., Govaerts, R., Vandewalle, J.: Hash functions based on block ciphers: A synthetic approach. In: Advances in Cryptology - CRYPTO '93. LNCS, vol. 773, pp. 368-378. Springer, Heidelberg (1993)

49. Rabin, M.: Digitalized signatures. In: Foundations of Secure Computation '78. pp. 155-166. Academic Press, New York (1978)

50. Rogaway, P., Shrimpton, T.: Cryptographic hash-function basics: Definitions, implications, and separations for preimage resistance, second-preimage resistance, and collision resistance. In: Fast Software Encryption 2004. LNCS, vol. 3017, pp. 371-388. Springer, Heidelberg (2004)

51. Rogaway, P., Steinberger, J.: Constructing cryptographic hash functions from fixed-key blockciphers. In: Advances in Cryptology - CRYPTO 2008. LNCS, vol. 5157, pp. 433-450. Springer, Heidelberg (2008)

52. Sasaki, Y.: Known-key attacks on Rijndael with large blocks and strengthening ShiftRow parameter. In: International Workshop on Security - IWSEC 2010. LNCS, vol. 6434, pp. 301-315. Springer, Heidelberg (2010)

53. Sasaki, Y., Emami, S., Hong, D., Kumar, A.: Improved known-key distinguishers on Feistel-SP ciphers and application to Camellia. In: Australasian Conference on Information Security and Privacy - ACISP 2012. LNCS, vol. 7372, pp. 87-100. Springer, Heidelberg (2012)

54. Sasaki, Y., Wang, L.: Distinguishers beyond three rounds of the RIPEMD-128/-160 compression functions. In: Applied Cryptography and Network Security 2012. LNCS, vol. 7341, pp. 275-292. Springer, Heidelberg (2012)

55. Sasaki, Y., Wang, L., Takasaki, Y., Sakiyama, K., Ohta, K.: Boomerang distinguishers for full HAS-160 compression function. In: International Workshop on Security - IWSEC 2012. LNCS, vol. 7631, pp. 156-169. Springer, Heidelberg (2012)

56. Sasaki, Y., Yasuda, K.: Known-key distinguishers on 11-round Feistel and collision attacks on its hashing modes. In: Fast Software Encryption 2011. LNCS, vol. 6733, pp. 397-415. Springer, Heidelberg (2011)

57. Shrimpton, T., Stam, M.: Building a collision-resistant compression function from noncompressing primitives. In: International Colloquium on Automata, Languages and Programming - ICALP (2) 2008. LNCS, vol. 5126, pp. 643-654. Springer, Heidelberg (2008)

58. Smith, J.: The design of Lucifer: a cryptographic device for data communications. IBM Research Report RC 3326 (1971)

59. Stam, M.: Blockcipher-based hashing revisited. In: Fast Software Encryption 2009. LNCS, vol. 5665, pp. 67-83. Springer, Heidelberg (2009)

60. Wagner, D.: The boomerang attack. In: Fast Software Encryption '99. LNCS, vol. 1636, pp. 156-170. Springer, Heidelberg (1999)

61. Yu, H., Chen, J., Wang, X.: The boomerang attacks on the round-reduced Skein-512. In: Selected Areas in Cryptography 2012. LNCS, vol. 7707, pp. 287-303. Springer, Heidelberg (2012)

\section{A Knudsen-Rijmen Attack on Feistel $_{7}$}

We briefly discuss the attack of Knudsen and Rijmen 27] on the classical Feistel network on $n$ bits with 7 rounds. Before doing so, we first introduce Feistel. 
The Feistel network is a very common blockcipher design strategy, dating back to the design of Lucifer [58], and many generalizations of this design have appeared in literature. We use the notation of [27]. The $n$-bit Feistel network with 7 rounds, called "Feistel ${ }_{7}$, " uses 2 lines of $n / 2$ bits, and consists of 7 evaluations of a fixed $n / 2$-bit permutation $p$. Each evaluation of $p$ is preceded by an XOR of a round key $k_{1}, \ldots, k_{7}$, derived from the master key using some key schedule. It is depicted in Fig. 5.

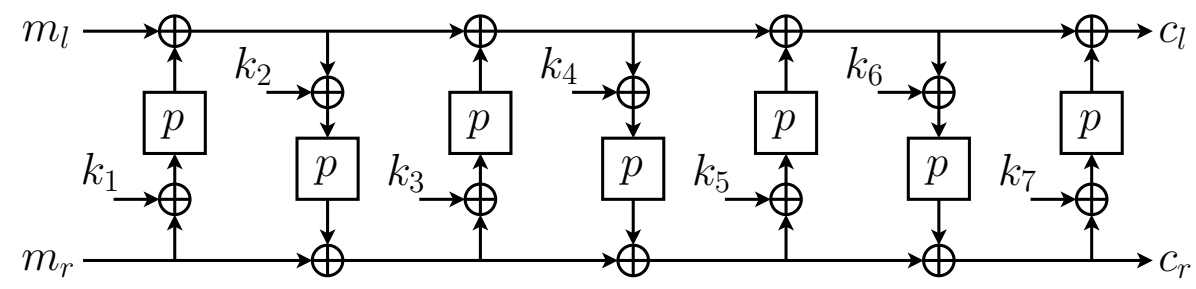

Fig. 5. The $n$-bit Feistel network with 7 rounds.

Now, we describe the attack by Knudsen and Rijmen on this construction [1,27]. Assume $k_{2} \neq k_{6}$. First, the adversary fixes an arbitrary value $y \in\{0,1\}^{n / 2}$. Then, from $y$ the adversary derives two plaintext/ciphertext-pairs $\left(\left(m_{l}, m_{r}\right),\left(c_{l}, c_{r}\right)\right)$ and $\left(\left(m_{l}^{\prime}, m_{r}^{\prime}\right),\left(c_{l}^{\prime}, c_{r}^{\prime}\right)\right)$ as follows: $y$ is fixed to be the input to the third permutation call $p$ for the first tuple, while for the second tuple that input is $y \oplus \alpha$ for some non-zero value $\alpha$ (determined later). Then, the adversary uses $p$ to compute the tuples in a straightforward way:

$$
\begin{aligned}
&\left(m_{l}, m_{r}\right)=\left(z \oplus k_{4} \oplus p(y) \oplus p\left(m_{r} \oplus k_{1}\right), y \oplus k_{3} \oplus p\left(z \oplus k_{2} \oplus k_{4} \oplus p(y)\right)\right) \\
&\left(c_{l}, c_{r}\right)=\left(z \oplus k_{2} \oplus k_{4} \oplus k_{6} \oplus p(y) \oplus p\left(c_{r} \oplus k_{7}\right),\right. \\
& y\left.y \oplus \oplus k_{5} \oplus p\left(z \oplus k_{2} \oplus k_{4} \oplus p(y)\right)\right) \\
&\left(m_{l}^{\prime}, m_{r}^{\prime}\right)=\left(z \oplus k_{2} \oplus k_{4} \oplus k_{6} \oplus p(y) \oplus p\left(m_{r}^{\prime} \oplus k_{1}\right),\right. \\
&\left.y \oplus \alpha \oplus k_{3} \oplus p\left(z \oplus k_{4} \oplus k_{6} \oplus p(y)\right)\right) \\
&\left(c_{l}^{\prime}, c_{r}^{\prime}\right)=\left(z \oplus k_{4} \oplus p(y) \oplus p\left(c_{r}^{\prime} \oplus k_{7}\right), y \oplus k_{5} \oplus p\left(z \oplus k_{4} \oplus k_{6} \oplus p(y)\right)\right),
\end{aligned}
$$

where $\alpha=y \oplus p^{-1}\left(k_{2} \oplus k_{6} \oplus p(y)\right)$ and $z=p^{-1}\left(k_{3} \oplus k_{5} \oplus \alpha\right)$. These pairs satisfy $m_{r} \oplus c_{r}=$ $m_{r}^{\prime} \oplus c_{r}^{\prime}$ with probability 1 , but this equation is satisfied by an ideal cipher with probability at most $1 / 2^{n / 2}$. This completes the distinguishing attack. An extension of this attack to generalized balanced Feistel networks on $r$ wires and $4 r-1$ rounds was derived in [1].

\section{B Proof of Theorem 2}

We focus on PGV2. The analysis for PGV1 is a simplification due to the absence of the feed-forward of the key. We consider any adversary that has query access to $\pi \stackrel{\$}{\leftarrow}$ $\mathrm{BC}\left[\Phi\left(A, B, \varphi^{C}\right)\right](n, n)$ and makes $q$ queries. Let $Z \in\{0,1\}^{n}$. A preimage for $Z$ would imply the existence of a query $(k, x, z)$ such that $x \oplus z=k \oplus Z$. We consider the $i^{\text {th }}$ query $(i \in\{1, \ldots, q\})$ to be the first query to make this condition satisfied, and sum over $i=1, \ldots, q$ at the end. For regular (forward or inverse) queries, the analysis of $[9,10,59$ mostly carries over. The analysis of predicate queries is a more technical, particularly as we cannot make use of abortable ciphers.

Query $\pi_{k}(x)$ or $\pi_{k}^{-1}(z)$. The cases are the same by symmetry, and we consider $\pi_{k}(x)$ only. Denote the response by $z$. As $z$ is randomly drawn from a set of size at least $2^{n}-B q$, it satisfies $z=x \oplus k \oplus Z$ with probability at most $\frac{1}{2^{n}-B q}$.

Query $\boldsymbol{\pi}_{\boldsymbol{k}}^{\boldsymbol{\Phi}}(\boldsymbol{y})$. Denote the query response by $\left\{\left(k, x^{1}, z^{1}\right), \ldots,\left(k, x^{B}, z^{B}\right)\right\}$. If all tuples are old, the query cannot be successful as no earlier query was successful, and so we assume it contains at least one new tuple. The response is drawn uniformly at random from the set 
$\Sigma_{k}\left(P_{k}, P_{k}^{\Phi}\right)$. For $\ell=0, \ldots, B$, denote by $\Sigma_{k}^{\ell}\left(P_{k}, P_{k}^{\Phi}\right)$ the subset of all responses that have $\ell$ new query tuples and $B-\ell$ old query tuples (which already appear in $P_{k}$ ). By construction,

$$
\Sigma_{k}\left(P_{k}, P_{k}^{\Phi}\right)=\bigcup_{\ell=0}^{B} \Sigma_{k}^{\ell}\left(P_{k}, P_{k}^{\Phi}\right) .
$$

Define furthermore for $\ell=1, \ldots, B$ by $\Sigma_{k}^{\ell, \text { pre }}\left(P_{k}, P_{k}^{\Phi}\right)$ the subset of elements of $\Sigma_{k}^{\ell}\left(P_{k}, P_{k}^{\Phi}\right)$ for which one of the new query tuples satisfies $x \oplus z=k \oplus Z$ (recall that we have excluded the case of $\ell=0$ ). The predicate query is successful with probability

$$
\operatorname{Pr}\left(\pi_{k}^{\Phi}(y) \text { sets } \operatorname{pre}\left(\mathcal{Q}_{i}\right)\right)=\sum_{\ell=1}^{B} \frac{\left|\Sigma_{k}^{\ell, \operatorname{pre}}\left(P_{k}, P_{k}^{\Phi}\right)\right|}{\left|\Sigma_{k}\left(P_{k}, P_{k}^{\Phi}\right)\right|}
$$

Using (10), we bound (11) as

$$
\operatorname{Pr}\left(\pi_{k}^{\Phi}(y) \text { sets } \operatorname{pre}\left(\mathcal{Q}_{i}\right)\right) \leq \frac{\left|\Sigma_{k}^{1, \text { pre }}\left(P_{k}, P_{k}^{\Phi}\right)\right|}{\left|\Sigma_{k}^{B}\left(P_{k}, P_{k}^{\Phi}\right)\right|}+\sum_{\ell=2}^{B} \frac{\left|\Sigma_{k}^{\ell, \text { pre }}\left(P_{k}, P_{k}^{\Phi}\right)\right|}{\left|\Sigma_{k}^{\ell}\left(P_{k}, P_{k}^{\Phi}\right)\right|}
$$

The reason why $\ell=1$ is treated differently, will become clear shortly.

We next bound all relevant sets. Here, for integers $a \geq b \geq 1$, we denote by $a^{\underline{b}}=\frac{a !}{(a-b) !}$ the falling factorial power. Starting with the numerators, for $\ell=1$ we have

$$
\left|\Sigma_{k}^{1, \operatorname{pre}}\left(P_{k}, P_{k}^{\Phi}\right)\right| \leq B \cdot\left|P_{k}\right| \frac{B-1}{3} \cdot\left(2^{n}-\left|P_{k}\right|\right) .
$$

Indeed, we have $B$ positions for the sole new query to appear and $\left|P_{k}\right| \frac{B-1}{}$ choices for the old queries. For the new query, without loss of generality $\left(k, x^{B}, z^{B}\right)$, it needs to satisfy $\operatorname{Bits}_{C}\left(x^{B} \oplus z^{B}\right)=\operatorname{Bits}_{C}\left(x^{1} \oplus \cdots \oplus z^{B-1}\right)$ and $x^{B} \oplus z^{B}=k \oplus Z$. We have $2^{n}-\left|P_{k}\right|$ possible choices for $x^{B}$, and any choice gives at most one possible $z^{B}$. We remark that $\left|\Sigma_{k}^{1, \text { pre }}\left(P_{k}, P_{k}^{\Phi}\right)\right|$ will probably be about a factor $2^{-|C|}$ less, as we should only count all possible solutions for the $B-1$ old queries that satisfy $\operatorname{Bits}_{C}\left(x^{1} \oplus \cdots \oplus z^{B-1}\right)=\operatorname{Bits}_{C}(k \oplus Z)$. Deriving a tighter bound would be a cumbersome exercise, but fortunately there is no need to do so: the fraction of elements in $\Sigma_{k}\left(P_{k}, P_{k}^{\Phi}\right)$ consisting of $B-1$ old tuples is already small enough for the case $B>1$. This is the reason why we use a special treatment for the case of $\ell=1$ in (12).

For $\ell \in\{2, \ldots, B\}$ we have

$$
\left|\Sigma_{k}^{\ell, \text { pre }}\left(P_{k}, P_{k}^{\Phi}\right)\right| \leq\left(\begin{array}{c}
B \\
\ell
\end{array}\right) \cdot\left|P_{k}\right| \frac{B-\ell}{} \cdot\left(2^{n}-\left|P_{k}\right|\right)^{\ell} \cdot \ell \cdot\left(2^{n}-\left|P_{k}\right|\right) \frac{\ell-2}{2} \cdot 2^{n-|C|} .
$$

Again, the first term comes from identifying at which positions the new queries appear and the second term comes from the selection of old queries. Next, we have $\left(2^{n}-\left|P_{k}\right|\right)^{\ell}$ choices for the $x$-values and $\ell$ positions for the "winning query" to occur. For this particular winning query, the corresponding $z$-value is fixed by the equation $x \oplus z=k \oplus Z$. For the remaining $\ell-1 z$-values, there are $\left(2^{n}-\left|P_{k}\right|\right)^{\frac{\ell-2}{}}$ possibilities to freely fix the first $\ell-2$ of them, and the last one will be adapted to the predicate condition, and can take at most $2^{n-|C|}$ values.

Regarding the denominators, for $\ell \in\{1, \ldots, B\}$ we have

$$
\left|\Sigma_{k}^{\ell}\left(P_{k}, P_{k}^{\Phi}\right)\right| \geq\left(\begin{array}{c}
B \\
\ell
\end{array}\right) \cdot\left|P_{k}\right| \frac{B-\ell}{\underline{B}} \cdot\left(\begin{array}{c}
\left(2^{n}-\left|P_{k}\right|\right)^{\underline{\ell}} \cdot\left(2^{n}-\left|P_{k}\right|\right)^{\ell-1} \cdot 2^{n-|C|}- \\
B q \cdot\left(2^{n}-\left|P_{k}\right|\right)^{\frac{\ell-1}{}} \cdot\left(2^{n}-\left|P_{k}\right|\right)^{\frac{\ell-1}{2}} \cdot 2^{n-|C|}
\end{array}\right),
$$

which can be seen as follows. As before, we have $\left(\begin{array}{c}B \\ \ell\end{array}\right)$ positions for the new queries to appear and $\left|P_{k}\right| \frac{B-\ell}{2}$ possible lists of old queries. Regarding the $\ell$ new queries, without loss of generality $\left(k, x^{1}, z^{1}\right), \ldots,\left(k, x^{\ell}, z^{\ell}\right)$, these need to satisfy $\operatorname{Bits}_{C}\left(x^{1} \oplus \cdots \oplus z^{\ell}\right)=\operatorname{Bits}_{C}\left(x^{\ell+1} \oplus\right.$ 
$\left.\cdots \oplus z^{B}\right)$. We first compute the number of choices for these new queries where $z^{\ell}$ is only used to adapt to this condition and does not need to satisfy that it is fresh. For this case, we have precisely $\left(2^{n}-\left|P_{k}\right|\right)^{\ell} \cdot\left(2^{n}-\left|P_{k}\right|\right)^{\frac{\ell-1}{2}}$ choices for $x^{1}, \ldots, z^{\ell-1}, x^{\ell}$, and $2^{n-|C|}$ possibilities for the adaption value $z^{\ell}$.

Now, we subtract the cases where this adapted value happens to collide, either with an older value in $\operatorname{nng}\left(P_{k}\right)$ or with any of the new $z^{1}, \ldots, z^{\ell-1}$. Any of these choices would fix $z^{\ell}$ (in total at most $\left(\left|P_{k}\right|+\ell-1\right)$ possibilities). Similarly to the analysis for $\left|\Sigma_{k}^{\ell, \text { pre }}\left(P_{k}, P_{k}^{\Phi}\right)\right|$, where now $x^{\ell}$ will be used to be adapted to the predicate condition, there are at most

$$
\left(\left|P_{k}\right|+\ell-1\right) \cdot\left(2^{n}-\left|P_{k}\right|\right) \frac{\ell-1}{2} \cdot\left(2^{n}-\left|P_{k}\right|\right)^{\frac{\ell-1}{2}} \cdot 2^{n-|C|}
$$

choices for the fresh values. As $\ell \leq B$, and additionally $\left|P_{k}\right| \leq B(i-1) \leq B(q-1)$ for the current query, we obtain our bound for $\left|\Sigma_{k}^{\ell}\left(P_{k}, P_{k}^{\Phi}\right)\right|$. The bound can be simplified to

$\left|\Sigma_{k}^{\ell}\left(P_{k}, P_{k}^{\Phi}\right)\right| \geq\left(\begin{array}{c}B \\ \ell\end{array}\right) \cdot\left|P_{k}\right| \frac{B-\ell}{2} \cdot\left(2^{n}-\left|P_{k}\right|\right)^{\ell-1} \cdot\left(2^{n}-\left|P_{k}\right|\right)^{\ell-1} \cdot 2^{n-|C|} \cdot\left(2^{n}-2 B q\right)$,

using that $\frac{\left(2^{n}-\left|P_{k}\right|\right)^{\underline{\ell}}}{\left(2^{n}-\left|P_{k}\right|\right)^{\ell-1}}=2^{n}-\left|P_{k}\right|-(\ell-1) \geq 2^{n}-B q$.

Plugging these bounds into 12 , we find for the case $B=1$ :

$$
\operatorname{Pr}\left(\pi_{k}^{\Phi}(y) \text { sets pre }\left(\mathcal{Q}_{i}\right)\right) \leq \frac{2^{n}-\left|P_{k}\right|}{2^{n-|C|} \cdot\left(2^{n}-2 q\right)} \leq \frac{2^{|C|}}{2^{n}-2 q} .
$$

For the case $B>1$ the computation is a bit more elaborate:

$$
\begin{aligned}
\operatorname{Pr}\left(\pi_{k}^{\Phi}(y) \text { sets pre }\left(\mathcal{Q}_{i}\right)\right) \leq & \frac{B \cdot\left(2^{n}-\left|P_{k}\right|\right)}{\left(2^{n}-\left|P_{k}\right|\right)^{\frac{B-1}{}} \cdot 2^{n-|C|} \cdot\left(2^{n}-2 B q\right)} \cdot \frac{\left|P_{k}\right| \frac{B-1}{\left(2^{n}-\left|P_{k}\right|\right)^{\frac{B-1}{B}}}+}{}+ \\
& \sum_{\ell=2}^{B} \frac{\left(2^{n}-\left|P_{k}\right|\right)^{\underline{\ell}} \cdot\left(2^{n}-\left|P_{k}\right|\right)^{\frac{\ell-2}{}}}{\left(2^{n}-\left|P_{k}\right|\right)^{\frac{\ell-1}{2}} \cdot\left(2^{n}-\left|P_{k}\right|\right)^{\frac{\ell-1}{}}} \cdot \frac{\ell}{2^{n}-2 B q} .
\end{aligned}
$$

For the first fraction we use that $2^{n}-\left|P_{k}\right| \leq\left(2^{n}-\left|P_{k}\right|\right) \frac{B-1}{\text { as } B>1 \text {, and additionally }}$ that $|C| \leq n$. For the falling factorial powers of the second fraction, we use that $\left|P_{k}\right| \frac{B-1}{} \leq$ $(B q)^{B-1}$ and $\left(2^{n}-\left|P_{k}\right|\right)^{B-1} \geq\left(2^{n}-\left|P_{k}\right|-(B-1)\right)^{B-1} \geq\left(2^{n}-2 B q\right)^{B-1}$. For the fraction

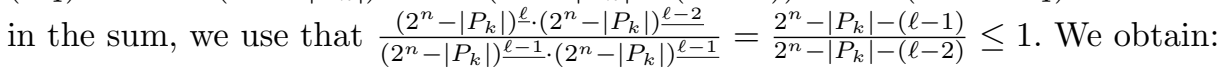

$$
\begin{aligned}
\operatorname{Pr}\left(\pi_{k}^{\Phi}(y) \text { sets pre }\left(\mathcal{Q}_{i}\right)\right) & \leq \frac{B}{2^{n}-2 B q} \cdot \frac{(B q)^{B-1}}{\left(2^{n}-2 B q\right)^{B-1}}+\sum_{\ell=2}^{B} \frac{\ell}{2^{n}-2 B q} \\
& \leq \frac{B^{B} q^{B-1}}{\left(2^{n}-2 B q\right)^{B}}+\frac{B^{2}}{2^{n}-2 B q} .
\end{aligned}
$$

Conclusion. Taking the maximum of all success probabilities, the $i^{\text {th }}$ query is successful with probability at most $\frac{B^{B} q^{B-1}}{\left(2^{n}-2 B q\right)^{B}}+\frac{B^{2} \delta_{B, C}[1]}{2^{n}-2 B q}$. Summation over $i=1, \ldots, q$ gives

$$
\operatorname{Adv}_{\mathrm{PGV} 2}^{\mathrm{epre}}(q) \leq \frac{B^{B} q^{B}}{\left(2^{n}-2 B q\right)^{B}}+\frac{B^{2} \delta_{B, C}[1] q}{2^{n}-2 B q}
$$

The proof is completed by using the fact that $2^{n}-2 B q \geq 2^{n-1}$ for $B q \leq 2^{n-2}$.

\section{Proof of Theorem 3}

We consider any adversary that has access to $\left(\pi_{1}, \pi_{2}\right) \stackrel{\$}{\leftarrow} \mathrm{BC}\left[\Phi\left(A, B, \varphi^{C}\right)\right](n)^{2}$ and makes $q$ queries. These queries are stored in a query history $\mathcal{Q}$ as indexed tuples of the form $\left(\kappa_{i}, x_{i}, z_{i}\right)$ 
for regular queries, where we denote $z_{i}=\pi_{\kappa_{i}}\left(x_{i}\right)$, and $\left\{\left(\kappa_{i}, x_{i}^{1}, z_{i}^{1}\right), \ldots,\left(\kappa_{i}, x_{i}^{B}, z_{i}^{B}\right)\right\}$ for predicate queries. For $q \geq 0$, by $\mathcal{Q}_{q}$ we define the query history after $q$ queries. As a first step, we move from $\left(\pi_{1}, \pi_{2}\right)$ to $\left(\bar{\pi}_{1}, \bar{\pi}_{2}\right) \stackrel{\$}{\leftarrow} \overline{\mathrm{BC}}\left[\Phi\left(A, B, \varphi^{C}\right)\right](n)^{2}$. By Lem. 2 , this costs us an additional term $\frac{B^{2} q(q+1)}{2^{n}-B q}$.

A collision for $\mathrm{F}_{\text {Grøstl }}^{\prime}$ would imply the existence of query pairs $\left(x_{1}, z_{1}\right),\left(x_{1}^{\prime}, z_{1}^{\prime}\right)$ for $\bar{\pi}_{1}$ and $\left(x_{2}, z_{2}\right),\left(x_{2}^{\prime}, z_{2}^{\prime}\right)$ for $\bar{\pi}_{2}$ such that $\left(x_{1}, x_{2}\right) \neq\left(x_{1}^{\prime}, x_{2}^{\prime}\right)$ and

$$
x_{1} \oplus z_{1} \oplus x_{2} \oplus z_{2}=x_{1}^{\prime} \oplus z_{1}^{\prime} \oplus x_{2}^{\prime} \oplus z_{2}^{\prime} .
$$

We define this configuration by $\operatorname{col}\left(\mathcal{Q}_{q}\right)$. For the analysis of $\operatorname{Pr}\left(\operatorname{col}\left(\mathcal{Q}_{q}\right)\right)$ for the case of $B>1$ we introduce an auxiliary event aux $\left(\mathcal{Q}_{q}\right)$. Let $\tau>0$ be any integral value. We define

$$
\operatorname{aux}\left(\mathcal{Q}_{q}\right): B>1 \wedge\left(\left|\operatorname{set}\left(\mathcal{Q}_{q}\right)\right|>q+\tau\right)
$$

where

$\operatorname{set}\left(\mathcal{Q}_{q}\right)=\left\{\left(\kappa, x_{i}, z_{i}\right),\left(\kappa, x_{j}, z_{j}\right) \in \mathcal{Q}_{q} \mid x_{i} \neq x_{j} \wedge \operatorname{Bits}_{C}\left(x_{i} \oplus z_{i} \oplus x_{j} \oplus z_{j}\right)=0\right\}$.

By basic probability theory:

$$
\operatorname{Adv}_{\mathrm{F}_{\text {Grøstl }}^{\prime}}^{\text {col }}(q) \leq \operatorname{Pr}\left(\operatorname{col}\left(\mathcal{Q}_{q}\right) \wedge \neg \operatorname{aux}\left(\mathcal{Q}_{q}\right)\right)+\operatorname{Pr}\left(\operatorname{aux}\left(\mathcal{Q}_{q}\right)\right)+\frac{B^{2} q(q+1)}{2^{n}-B q},
$$

where the third part of the bound comes from the transition from WCM to AWCM.

Starting with $\operatorname{Pr}\left(\operatorname{aux}\left(\mathcal{Q}_{q}\right)\right)$, we make a distinction between $B=2$ and $B \geq 3$. For $B=2$, we make a further distinction depending on whether or not $\left(\kappa, x_{i}, z_{i}\right),\left(\kappa, x_{j}, z_{j}\right)$ come from the same predicate query. Clearly, if this is the case, they satisfy the condition $\operatorname{Bits}_{C}\left(x_{i} \oplus z_{i} \oplus x_{j} \oplus z_{j}\right)=0$ by design. As the adversary makes at most $q$ predicate queries, $\operatorname{set}\left(\mathcal{Q}_{q}\right)$ will contain at most $q$ solutions of this kind. Regarding the case where the two queries are not from the same predicate query (i.e., they are from two different predicate queries or at least one of them comes from a regular query), any combination of two tuples satisfies Bits $C_{C}\left(x_{i} \oplus z_{i} \oplus x_{j} \oplus z_{j}\right)=0$ with probability $2^{-|C|}$. Consequently, as there are at most $\left(\begin{array}{c}B q \\ 2\end{array}\right)$ such combinations,

$$
\operatorname{Ex}\left(\left|\operatorname{set}\left(\mathcal{Q}_{q}\right)\right|-q\right) \leq \frac{\left(\begin{array}{c}
B q \\
2
\end{array}\right)}{2^{|C|}}
$$

Markov's inequality states that

$$
\operatorname{Pr}\left(\operatorname{aux}\left(\mathcal{Q}_{q}\right)\right) \leq \frac{\left(\begin{array}{c}
B q \\
2
\end{array}\right)}{\tau 2^{|C|}}
$$

For the case of $B \geq 3$, the same bound applies with the difference that the case " $\left(\kappa, x_{i}, z_{i}\right)$, $\left(\kappa, x_{j}, z_{j}\right)$ coming from the same predicate query" does not render a solution by design but is instead counted within part two of the analysis (it is already included in the $\left(\begin{array}{c}B q \\ 2\end{array}\right)$ ).

We proceed with the analysis of $\operatorname{Pr}\left(\operatorname{col}\left(\mathcal{Q}_{q}\right) \wedge \neg \operatorname{aux}\left(\mathcal{Q}_{q}\right)\right)$. We consider the $i^{\text {th }}$ query $(i \in$ $\{1, \ldots, q\})$ to be the first query to make this condition satisfied, and sum over $i=1, \ldots, q$ at the end. For regular (forward or inverse) queries, the analysis of 20] mostly carries over. The analysis of predicate queries is a bit more technical.

Regular (forward or inverse) query. The cases are the same by symmetry, and we consider $\bar{\pi}_{1}\left(x_{1}\right)$ only. Denote the query response by $z_{1}$. There are at most $B(i-1)$ possible choices for each of $\left(x_{1}^{\prime}, z_{1}^{\prime}\right),\left(x_{2}, z_{2}\right)$, and $\left(x_{2}^{\prime}, z_{2}^{\prime}\right)$, where $\left(x_{2}, z_{2}\right)$ and $\left(x_{2}^{\prime}, z_{2}^{\prime}\right)$ may be the same but the choice of $\left(x_{1}^{\prime}, z_{1}^{\prime}\right)$ is necessarily older than the current query. As $z_{1}$ is randomly drawn from a set of size at least $2^{n}-B q$, it satisfies 13 with probability at most $\frac{B^{3}(i-1)^{3}}{2^{n}-B q}$. 
Query $\overline{\boldsymbol{\pi}}_{1}^{\Phi}(\boldsymbol{y})$ or $\overline{\boldsymbol{\pi}}_{2}^{\Phi}(\boldsymbol{y})$. The cases are the same by symmetry, and we consider $\bar{\pi}_{1}^{\Phi}(y)$ only. Denote the query response by $\left\{\left(x_{1}^{1}, z_{1}^{1}\right), \ldots,\left(x_{1}^{B}, z_{1}^{B}\right)\right\}$. In case the $B$-set contributes only to $\left(x_{1}, z_{1}\right)$, the same reasoning as for regular queries applies with the difference that any query of the $B$-set may be successful and that the bound of Lem. 2 part (ii) applies: $\frac{B^{4} \delta_{B, C}[1](i-1)^{3}}{2^{n}-B q}$.

Now, consider the case the predicate query contributes to both $\left(x_{1}, z_{1}\right)$ and $\left(x_{1}^{\prime}, z_{1}^{\prime}\right)$. There are $\left(\begin{array}{c}B \\ 2\end{array}\right)$ ways for the predicate query to contribute (or 0 if $B=1$ ). By $\neg$ aux $\left(\mathcal{Q}_{q}\right)$, there are at most $q+\tau$ choices for $\left(x_{2}, z_{2}\right),\left(x_{2}^{\prime}, z_{2}^{\prime}\right)$ to satisfy $\operatorname{Bits}_{C}\left(x_{2} \oplus z_{2} \oplus x_{2}^{\prime} \oplus z_{2}^{\prime}\right)=0$. By Lem. 2 part (iii), the predicate query gives a solution to (13) with probability at most $\left(\begin{array}{l}B \\ 2\end{array}\right) \frac{\delta_{B, C}[2](q+\tau) 2^{n}}{2^{2 n}-B q}$.

Conclusion. Taking the maximum of all success probabilities, the $i^{\text {th }}$ query is successful with probability at most $\frac{B^{4} \delta_{B, C}[1](i-1)^{3}}{2^{n}-B q}+\left(\begin{array}{c}B \\ 2\end{array}\right) \frac{\delta_{B, C}[2](q+\tau) 2^{n}}{2^{2 n}-B q}$. Summation over $i=1, \ldots, q$ gives, using (14),

$$
\mathbf{A d v}_{\mathrm{F}_{\mathrm{Gr} r s \mathrm{st}}}^{\text {col }}(q) \leq \frac{B^{4} \delta_{B, C}[1] q^{4}}{2\left(2^{n}-B q\right)}+\left(\begin{array}{c}
B \\
2
\end{array}\right) \frac{\delta_{B, C}[2]\left(q^{2}+\tau q\right) 2^{n}}{2^{2 n}-B q}+\frac{\left(\begin{array}{c}
B q \\
2
\end{array}\right)}{\tau 2^{|C|}}+\frac{B^{2} q(q+1)}{2^{n}-B q}
$$

The value $\tau$ forms a threshold between the second and third fraction (the second fraction increases while the third fraction decreases for larger $\tau$, and vice versa for smaller $\tau$ ). We put $\tau=2^{n / 2-|C|}$. The proof is completed by using the fact that $2^{n}-B q \geq 2^{n-1}$ for $B q \leq 2^{n-1}$, and that $q+1 \leq 2 q$ for $q \geq 1$.

\section{Tightness of the Bound of Theorem 3}

For the cases $B=1$ and $C$ arbitrary, and $B=2$ and $C$ arbitrary such that $|C|>n / 2$, we derive generic attacks that demonstrate tightness of the bound of Thm. 3 . Mendel et al. 38 already exploited a known-key pair for the underlying permutations of Grøstl to find a semi-free-start collision with a lower complexity, but we approach the problem more generically, particularly considering arbitrary but independent $\pi_{1}$ and $\pi_{2}$.

Proposition $4(\boldsymbol{B}=\mathbf{1})$. Let $n \in \mathbb{N}$. Suppose $\left(\pi_{1}, \pi_{2}\right) \stackrel{\&}{\leftarrow} \mathrm{BC}\left[\Phi\left(A, 1, \varphi^{C}\right)\right](n)^{2}$. Then, $\operatorname{Adv}_{\mathbf{F}_{\text {Grøst }}^{\prime}}^{\text {col }}(2 q) \geq \frac{q^{4}}{2^{n-|C|}}$.

Proof. We construct a collision-finding adversary $\mathcal{A}$ for $\mathrm{F}_{\text {Grøstl }}^{\prime}$. It makes predicate queries to $\pi_{1}^{\Phi}$ on input of distinct values $y$ to obtain $q$ queries $\left(1, x_{1, y}, z_{1, y}\right)$ satisfying $\operatorname{Bits}_{C}\left(x_{1, y} \oplus z_{1, y}\right)=$ 0 , and likewise for $\pi_{2}^{\Phi}$ it obtains $q$ queries $\left(2, x_{2, y}, z_{2, y}\right)$. Any two queries to $\pi_{1}^{\Phi}$ and two queries to $\pi_{2}^{\Phi}$ collide on the entire state, $x_{1, y} \oplus z_{1, y} \oplus x_{2, y} \oplus z_{2, y}=x_{1, y^{\prime}} \oplus z_{1, y^{\prime}} \oplus x_{2, y^{\prime}} \oplus z_{2, y^{\prime}}$, with

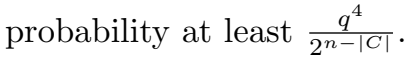

Proposition $5(B=2$ and $|\boldsymbol{C}|>\boldsymbol{n} / \mathbf{2})$. Let $n \in \mathbb{N}$. Suppose $\left(\pi_{1}, \pi_{2}\right) \stackrel{\$}{\leftarrow}$ $\operatorname{BC}\left[\Phi\left(A, 2, \varphi^{C}\right)\right](n)^{2}$. Then, $\mathbf{A d v}_{\mathbf{F}_{\mathrm{Gr} r s t 1}^{\prime}}^{\text {col }}(2 q) \geq \frac{q^{2}}{2^{n-|C|}}$.

Proof. We construct a collision-finding adversary $\mathcal{A}$ for $\mathrm{F}_{\text {Grøstl }}^{\prime}$. It makes predicate queries to $\pi_{1}^{\Phi}$ on input of distinct values $y$ to obtain $q 2$-sets $\left\{\left(1, x_{1, y}^{1}, z_{1, y}^{1}\right),\left(1, x_{1, y}^{2}, z_{1, y}^{2}\right)\right\}$ satisfying $\operatorname{Bits}_{C}\left(x_{1, y}^{1} \oplus z_{1, y}^{1}\right)=\operatorname{Bits}_{C}\left(x_{1, y}^{2} \oplus z_{1, y}^{2}\right)$, and likewise for $\pi_{2}^{\Phi}$ it obtains $q$ 2-sets $\left\{\left(2, x_{2, y}^{1}, z_{2, y}^{1}\right),\left(2, x_{2, y}^{2}, z_{2, y}^{2}\right)\right\}$. Any 2 -set from $\pi_{1}^{\Phi}$ and 2 -set from $\pi_{2}^{\Phi}$ collide on the entire state, $x_{1, y}^{1} \oplus z_{1, y}^{1} \oplus x_{2, y}^{1} \oplus z_{2, y}^{1}=x_{1, y}^{2} \oplus z_{1, y}^{2} \oplus x_{2, y}^{2} \oplus z_{2, y}^{2}$, with probability at least $\frac{q^{2}}{2^{n-|C|}}$.

\section{E Proof of Theorem 4}

We consider any adversary that has access to $\left(\pi_{1}, \pi_{2}\right) \stackrel{\$}{\leftarrow} \mathrm{BC}\left[\Phi\left(A, B, \varphi^{C}\right)\right](n)^{2}$ and makes $q$ queries. As in App. C] these queries are stored in a query history $\mathcal{Q}$ as indexed tuples of the 
form $\left(\kappa_{i}, x_{i}, z_{i}\right)$ for regular queries and $\left\{\left(\kappa_{i}, x_{i}^{1}, z_{i}^{1}\right), \ldots,\left(\kappa_{i}, x_{i}^{B}, z_{i}^{B}\right)\right\}$ for predicate queries. We use the same approach as in App. C. In particular, we make the same transition from $\left(\pi_{1}, \pi_{2}\right)$ to $\left(\bar{\pi}_{1}, \bar{\pi}_{2}\right) \stackrel{\$}{\leftarrow} \overline{\mathrm{BC}}\left[\Phi\left(A, B, \varphi^{C}\right)\right](n)^{2}$.

Let $Z \in\{0,1\}^{n}$. A preimage for $Z$ would imply the existence of query pairs $\left(x_{1}, z_{1}\right)$ for $\bar{\pi}_{1}$ and $\left(x_{2}, z_{2}\right)$ for $\bar{\pi}_{2}$ such that

$$
x_{1} \oplus z_{1} \oplus x_{2} \oplus z_{2}=Z
$$

We define this configuration by $\operatorname{pre}\left(\mathcal{Q}_{q}\right)$. For the analysis of $\operatorname{Pr}\left(\operatorname{pre}\left(\mathcal{Q}_{q}\right)\right)$ we introduce an auxiliary event $\operatorname{aux}\left(\mathcal{Q}_{q}\right)$. Let $\tau>0$ be any integral value. We define

$$
\operatorname{aux}\left(\mathcal{Q}_{q}\right):\left|\operatorname{set}\left(\mathcal{Q}_{q}\right)\right|>q+\tau,
$$

where

$$
\operatorname{set}\left(\mathcal{Q}_{q}\right)=\left\{\left(\kappa, x_{i}, z_{i}\right) \in \mathcal{Q}_{q} \mid \operatorname{Bits}_{C}\left(x_{i} \oplus z_{i}\right)=0\right\}
$$

By basic probability theory:

$$
\mathbf{A d v}_{\mathbf{F}_{\text {Grøstl }}^{\prime}}^{\text {epre }}(q) \leq \operatorname{Pr}\left(\operatorname{pre}\left(\mathcal{Q}_{q}\right) \wedge \neg \operatorname{aux}\left(\mathcal{Q}_{q}\right)\right)+\operatorname{Pr}\left(\operatorname{aux}\left(\mathcal{Q}_{q}\right)\right)+\frac{B^{2} q(q+1)}{2^{n}-B q},
$$

where the third part of the bound comes from the transition from WCM to AWCM.

Starting with $\operatorname{Pr}\left(\operatorname{aux}\left(\mathcal{Q}_{q}\right)\right)$, an identical analysis to the case of the collision resistance of $\mathrm{F}_{\text {Grøstl }}^{\prime}$ in App. C gives

$$
\operatorname{Pr}\left(\operatorname{aux}\left(\mathcal{Q}_{q}\right)\right) \leq \frac{B q}{\tau 2^{|C|}}
$$

We proceed with the analysis of $\operatorname{Pr}\left(\operatorname{pre}\left(\mathcal{Q}_{q}\right) \wedge \neg \operatorname{aux}\left(\mathcal{Q}_{q}\right)\right)$. We consider the $i^{\text {th }}$ query $(i \in$ $\{1, \ldots, q\})$ to be the first query to make this condition satisfied, and sum over $i=1, \ldots, q$ at the end. For regular (forward or inverse) queries, the analysis of 20 mostly carries over. The analysis of predicate queries is a bit more technical.

Regular (forward or inverse) query. The cases are the same by symmetry, and we consider $\bar{\pi}_{1}\left(x_{1}\right)$ only. Denote the query response by $z_{1}$. There are at most $B(i-1)$ possible choices for $\left(x_{2}, z_{2}\right)$. As $z_{1}$ is randomly drawn from a set of size at least $2^{n}-B q$, it satisfies 15 with probability at most $\frac{B(i-1)}{2^{n}-B q}$.

Query $\overline{\boldsymbol{\pi}}_{\mathbf{1}}^{\boldsymbol{\Phi}}(\boldsymbol{y})$ or $\overline{\boldsymbol{\pi}}_{\mathbf{2}}^{\boldsymbol{\Phi}}(\boldsymbol{y})$. The cases are the same by symmetry, and we consider $\bar{\pi}_{1}^{\Phi}(y)$ only. Denote the query response by $\left\{\left(x_{1}^{1}, z_{1}^{1}\right), \ldots,\left(x_{1}^{B}, z_{1}^{B}\right)\right\}$. We make a distinction between $B=1$ and $B>1$. First, if $B>1$, the same reasoning as for regular queries applies with the difference that any query of the $B$-set may be successful and that the bound of Lem. 2. part (ii) applies: $[B>1] \frac{B^{2} \delta_{B, C}[1](i-1)}{2^{n}-B q}$. Now, consider $B=1$. By $\neg \operatorname{aux}\left(\mathcal{Q}_{q}\right)$, there are at most $q+\tau$ choices for $\left(x_{2}, z_{2}\right)$ to satisfy $\operatorname{Bits}_{C}\left(x_{2} \oplus z_{2}\right)=0$. By Lem.2 part (ii), the predicate gives a solution to 15 with probability at most $[B=1] \frac{\delta_{B, C}[1](q+\tau)}{2^{n}-B q}$. Combining, the predicate query succeeds with probability at most $\frac{B^{2} \delta_{B, C}[1](q+\tau)}{2^{n}-B q}$.

Conclusion. Taking the maximum of all success probabilities, the $i^{\text {th }}$ query is successful with probability at most $\frac{B^{2} \delta_{B, C}[1](q+\tau)}{2^{n}-B q}$. Summation over $i=1, \ldots, q$ gives, using 16 ,

$$
\mathbf{A d v}_{\mathrm{F}_{\text {Grøstl }}^{\prime}}^{\text {epre }}(q) \leq \frac{B^{2} \delta_{B, C}[1]\left(q^{2}+\tau q\right)}{2^{n}-B q}+\frac{B q}{\tau 2^{|C|}}+\frac{B^{2} q(q+1)}{2^{n}-B q}
$$

We put threshold $\tau=2^{n / 2-|C|}$. The proof is completed by using the fact that $2^{n}-B q \geq 2^{n-1}$ for $B q \leq 2^{n-1}$, and that $q+1 \leq 2 q$ for $q \geq 1$. 


\section{F Tightness of the Bound of Theorem 4}

For the case $B=1$, we derive a generic attack that demonstrates the tightness of the bound of Thm. 4 .

Proposition $6(\boldsymbol{B}=\mathbf{1})$. Let $n \in \mathbb{N}$. Suppose $\left(\pi_{1}, \pi_{2}\right) \stackrel{\$}{\leftarrow} \mathrm{BC}\left[\Phi\left(A, 1, \varphi^{C}\right)\right](n)^{2}$. Then, $\mathbf{A d v}_{\mathbf{F}_{\text {Grøst }}^{\prime}}^{\text {epre }}(2 q) \geq \frac{q^{2}}{2^{n-|C|}}$.

Proof. Let $Z$ be any given range value with $\operatorname{Bits}_{C}(Z)=0$ (note that epre guarantees security for every range point). A preimage-finding adversary $\mathcal{A}$ for $\mathrm{F}_{\text {Grøstl }}^{\prime}$ proceeds as follows. It makes predicate queries to $\pi_{1}^{\Phi}$ on input of distinct values $y$ to obtain $q$ queries $\left(1, x_{1, y}, z_{1, y}\right)$ satisfying $\operatorname{Bits}_{C}\left(x_{1, y} \oplus z_{1, y}\right)=0$, and likewise for $\pi_{2}^{\Phi}$ it obtains $q$ queries $\left(2, x_{2, y}, z_{2, y}\right)$. Any query to $\pi_{1}^{\Phi}$ and query to $\pi_{2}^{\Phi}$ collide with $Z$ on the entire state, $x_{1, y} \oplus z_{1, y} \oplus x_{2, y} \oplus z_{2, y}=Z$,

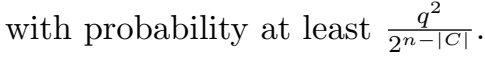

\section{G Proof of Theorem 5}

The bulk of the proof is captured in the following proposition.

Proposition 7. Let $n \in \mathbb{N}$. Suppose $\left(\pi_{1}, \pi_{2}, \pi_{3}\right) \stackrel{\$}{\leftarrow} \mathrm{BC}\left[\Phi\left(A, B, \varphi^{C}\right)\right](n)^{3}$. Then, for $q \leq$ $2^{n-1} / B$ and any positive integral value $\tau$,

$$
\begin{aligned}
\operatorname{Adv}_{\mathrm{F}_{\mathrm{SS}}}^{\mathrm{col}}(q) \leq & \frac{2 \tau^{3} B^{2}(B+2) \delta_{B, C}[1] q^{2}+4 B^{2} q^{2}+e^{2} B^{2}\left(\delta_{B, C}[1]\right)^{2} 2^{-|C|} q^{2}+e^{2} B^{2} q^{2}}{2^{n}}+ \\
& \left(\begin{array}{c}
B \\
2
\end{array}\right)\left[\frac{2 \tau^{2} \delta_{B, C}[2] q}{2^{n}}+\frac{2 \tau B^{2} \delta_{B, C}[2] q^{3}}{2^{2 n}}+\frac{2 B^{4} \delta_{B, C}[2] q^{5}}{2^{3 n}}\right]+ \\
& 2^{n}\left(\frac{e\left(B+\left(\begin{array}{c}
B \\
2
\end{array}\right)\right) q}{\tau} \cdot \frac{2 B^{2} \delta_{B, C}[1] q+2 \delta_{B, C}[2]}{2^{n}}\right)^{\tau /\left(B+\left(\begin{array}{c}
B \\
2
\end{array}\right)\right)}+ \\
& 2^{2 n}\left(\frac{2 e B^{3} \delta_{B, C}[1] q^{2}}{\tau 2^{n}}\right)^{\tau / B}+2^{n}\left(\frac{2 e B^{3} \delta_{B, C}[1] q^{2}}{2^{n}}+\frac{e B^{3} \delta_{B, C}[2] q^{3}}{\tau 2^{2 n}}\right)^{2 \tau / B} .
\end{aligned}
$$

The proof follows later in the appendix, but we first prove Thm. 5 using Prop. 7

\section{Proof of Theorem 5}

(i). We put $\tau=n$. Then, for the particular choice of $B$ and $C$ the bound (17) of Prop. 7 simplifies to

$$
\mathbf{A d v}_{\mathrm{F}_{\mathrm{SS}}}^{\mathrm{col}}(q) \leq \frac{6 n^{3} 2^{|C|} q^{2}+4 q^{2}+e^{2} 2^{|C|} q^{2}+e^{2} q^{2}}{2^{n}}+2 \cdot 2^{2 n}\left(\frac{2 e 2^{|C|} q^{2}}{2^{n}}\right)^{n}
$$

For $q=2^{(n-|C|) / 2-n \varepsilon}$, it is clear that all terms approach 0 for $n \rightarrow \infty$.

(ii) and (iii). We put $\tau=n$. Then, for the particular choice of $B$ (with $C$ arbitrary) the bound (17) of Prop. 7 simplifies to

$$
\begin{aligned}
\mathbf{A d v}_{\mathrm{F}_{\mathrm{SS}}}^{\mathrm{col}}(q) \leq & \frac{32 n^{3} q^{2}+16 q^{2}+8 e^{2} q^{2}}{2^{n}}+\frac{2 n^{2} 2^{|C|} q}{2^{n}}+\frac{8 n 2^{|C|} q^{3}}{2^{2 n}}+\frac{32 \cdot 2^{|C|} q^{5}}{2^{3 n}}+ \\
& 2^{n}\left(\frac{24 e q^{2}+6 e 2^{|C|} q}{n 2^{n}}\right)^{n / 3}+2^{2 n}\left(\frac{16 e q^{2}}{n 2^{n}}\right)^{n / 2}+2^{n}\left(\frac{16 e q^{2}}{2^{n}}+\frac{8 e 2^{|C|} q^{3}}{n 2^{2 n}}\right)^{n} .
\end{aligned}
$$

For $q=2^{n / 2-n \varepsilon}$ (in case $|C| \leq n / 2$ ), and for $q=2^{n-|C|-n \varepsilon}$ (in case $|C|>n / 2$ ), it is clear that all terms approach 0 for $n \rightarrow \infty$.

(iv). The reasoning of previous cases carries over, and we will not go into detail. Note that we have $\delta_{B, C}[1]=\delta_{B, C}[2]=1$ for $B \geq 3$. 


\section{Proof of Proposition 7}

We consider any adversary that has access to $\left(\pi_{1}, \pi_{2}, \pi_{3}\right) \stackrel{\&}{\leftarrow} \mathrm{BC}\left[\Phi\left(A, B, \varphi^{C}\right)\right](n)^{3}$ and makes $q$ queries. These queries are stored in a query history $\mathcal{Q}$ as indexed tuples of the form $\left(\kappa_{i}, x_{i}, z_{i}\right)$ for regular queries, where we denote $z_{i}=\pi_{\kappa_{i}}\left(x_{i}\right)$, and $\left\{\left(\kappa_{i}, x_{i}^{1}, z_{i}^{1}\right), \ldots\right.$, $\left.\left(\kappa_{i}, x_{i}^{B}, z_{i}^{B}\right)\right\}$ for predicate queries. For $q \geq 0$, by $\mathcal{Q}_{q}$ we define the query history after $q$ queries. As a first step, we move from $\left(\pi_{1}, \pi_{2}, \pi_{3}\right)$ to $\left(\bar{\pi}_{1}, \bar{\pi}_{2}, \bar{\pi}_{3}\right) \stackrel{\$}{\leftarrow} \overline{\mathrm{BC}}\left[\Phi\left(A, B, \varphi^{C}\right)\right](n)^{3}$. By Lem. 2. this costs us an additional term $\frac{B^{2} q(q+1)}{2^{n}-B q}$.

The adversary's goal is to find a collision for $\mathrm{F}_{\mathrm{SS}}$, which corresponds to obtaining query pairs $\left(x_{1}, z_{1}\right),\left(x_{1}^{\prime}, z_{1}^{\prime}\right)$ for $\bar{\pi}_{1},\left(x_{2}, z_{2}\right),\left(x_{2}^{\prime}, z_{2}^{\prime}\right)$ for $\bar{\pi}_{2}$, and $\left(x_{3}, z_{3}\right),\left(x_{3}^{\prime}, z_{3}^{\prime}\right)$ for $\bar{\pi}_{3}$ in the query history, such that:

$$
\begin{aligned}
\left(x_{1}, x_{2}\right) & \neq\left(x_{1}^{\prime}, x_{2}^{\prime}\right), \\
x_{1} \oplus z_{1} \oplus x_{2} \oplus z_{2} & =x_{3}, \\
x_{1}^{\prime} \oplus z_{1}^{\prime} \oplus x_{2}^{\prime} \oplus z_{2}^{\prime} & =x_{3}^{\prime}, \\
x_{1} \oplus z_{1} \oplus z_{3} & =x_{1}^{\prime} \oplus z_{1}^{\prime} \oplus z_{3}^{\prime} .
\end{aligned}
$$

We define this configuration by $\operatorname{col}\left(\mathcal{Q}_{q}\right)$. This means:

$$
\operatorname{Adv}_{\mathrm{F}_{\mathrm{SS}}}^{\mathrm{col}}(q)=\operatorname{Pr}\left(\operatorname{col}\left(\mathcal{Q}_{q}\right)\right)+\frac{B^{2} q(q+1)}{2^{n}-B q},
$$

where the second part of the bound comes from the transition from WCM to AWCM.

For the analysis of $\operatorname{Pr}\left(\operatorname{col}\left(\mathcal{Q}_{q}\right)\right)$ we introduce an auxiliary event aux $\left(\mathcal{Q}_{q}\right)$. Let $\tau_{2}, \tau_{3}, \tau_{4}$, $\tau_{5}>0$ be any integral values. We define $\operatorname{aux}\left(\mathcal{Q}_{q}\right)=\operatorname{aux}_{1 \vee \cdots \vee 5}\left(\mathcal{Q}_{q}\right)$, where

$$
\begin{aligned}
& \operatorname{aux}_{1}\left(\mathcal{Q}_{q}\right):\left|\left\{\begin{array}{c}
\left\{\left(\kappa_{i}, x_{i}^{1}, z_{i}^{1}\right), \ldots,\left(\kappa_{i}, x_{i}^{B}, z_{i}^{B}\right)\right\} \in \mathcal{Q}_{q}, \\
a, b \in\{1, \ldots, B\}, a \neq b \mid x_{i}^{a} \oplus z_{i}^{a}=x_{i}^{b} \oplus z_{i}^{b}
\end{array}\right\}\right|>0 ; \\
& \operatorname{aux}_{2}\left(\mathcal{Q}_{q}\right): \max _{Z \in\{0,1\}^{n}}\left|\left\{\left(\kappa_{i}, x_{i}, z_{i}\right) \in \mathcal{Q}_{q} \mid x_{i} \oplus z_{i}=Z\right\}\right|>\tau_{2} ; \\
& \operatorname{aux}_{3}\left(\mathcal{Q}_{q}\right): \max _{Z \in\{0,1\}^{n}}\left|\left\{\begin{array}{c}
\left(\kappa_{i}, x_{i}, z_{i}\right),\left(\kappa_{j}, x_{j}, z_{j}\right) \in \mathcal{Q}_{q} \mid \\
\left(\kappa_{i}, x_{i}\right) \neq\left(\kappa_{j}, x_{j}\right) \wedge x_{i} \oplus z_{i} \oplus x_{j} \oplus z_{j}=Z
\end{array}\right\}\right|>\tau_{3} ; \\
& \operatorname{aux}_{4}\left(\mathcal{Q}_{q}\right): \max _{Z, Z^{\prime} \in\{0,1\}^{n}}\left|\left\{\begin{array}{c}
\left(1, x_{i}, z_{i}\right),\left(2, x_{k}, z_{k}\right),\left(3, x_{l}, z_{l}\right) \in \mathcal{Q}_{q} \mid \\
x_{k} \oplus z_{k} \oplus x_{l}=Z^{\prime} \wedge x_{i} \oplus z_{i} \oplus x_{k} \oplus z_{k} \oplus x_{l} \oplus z_{l}=Z
\end{array}\right\}\right|>\tau_{4} ; \\
& \operatorname{aux}_{5}\left(\mathcal{Q}_{q}\right): \max _{Z \in\{0,1\}^{n}}\left|\left\{\begin{array}{c}
\left(1, x_{i}, z_{i}\right),\left(1, x_{j}, z_{j}\right),\left(2, x_{k}, z_{k}\right),\left(3, x_{l}, z_{l}\right) \in \mathcal{Q}_{q} \mid x_{i} \neq x_{j} \wedge \\
x_{j} \oplus z_{j} \oplus x_{k} \oplus z_{k} \oplus x_{l}=0 \wedge x_{i} \oplus z_{i} \oplus x_{k} \oplus z_{k} \oplus x_{l} \oplus z_{l}=Z
\end{array}\right\}\right|>\tau_{5} .
\end{aligned}
$$

We note that $\operatorname{aux}_{1}\left(\mathcal{Q}_{q}\right)$ only applies to predicate queries, $\operatorname{but} \operatorname{aux}_{2}\left(\mathcal{Q}_{q}\right), \ldots, \operatorname{aux}_{5}\left(\mathcal{Q}_{q}\right)$ apply to regular queries and parts of predicate queries (abusing notation). By basic probability theory, we obtain for (19):

$$
\operatorname{Pr}\left(\operatorname{col}\left(\mathcal{Q}_{q}\right)\right) \leq \operatorname{Pr}\left(\operatorname{col}\left(\mathcal{Q}_{q}\right) \wedge \neg \operatorname{aux}\left(\mathcal{Q}_{q}\right)\right)+\operatorname{Pr}\left(\operatorname{aux}\left(\mathcal{Q}_{q}\right)\right) .
$$

We start with the analysis of $\operatorname{Pr}\left(\operatorname{col}\left(\mathcal{Q}_{q}\right) \wedge \neg \operatorname{aux}\left(\mathcal{Q}_{q}\right)\right)$. For obtaining a query history that fulfills configuration $\operatorname{col}\left(\mathcal{Q}_{q}\right)$, it may be the case that a query appears at multiple positions. For instance, $x_{1}=x_{1}^{\prime}$. We split the analysis of $\operatorname{col}\left(\mathcal{Q}_{q}\right)$ into essentially all different possible cases. We define for binary $\alpha_{1}, \alpha_{2}, \alpha_{3}$ by $\operatorname{col}_{\alpha_{1} \alpha_{2} \alpha_{3}}(\mathcal{Q})$ the configuration $\operatorname{col}(\mathcal{Q})$ restricted to

$$
x_{1}=x_{1}^{\prime} \equiv \alpha_{1}, \quad x_{2}=x_{2}^{\prime} \equiv \alpha_{2}, \quad x_{3}=x_{3}^{\prime} \equiv \alpha_{3} .
$$

By construction, $\operatorname{col}\left(\mathcal{Q}_{q}\right) \Rightarrow \bigvee_{\alpha_{1}, \alpha_{2}, \alpha_{3} \in\{0,1\}} \operatorname{col}_{\alpha_{1} \alpha_{2} \alpha_{3}}\left(\mathcal{Q}_{q}\right)$, and from 19200 we obtain the following bound on $\operatorname{Adv}_{\mathrm{F}_{\mathrm{SS}}}^{\mathrm{col}}(q)$ :

$$
\operatorname{Adv}_{\mathrm{F}_{\mathrm{SS}}}^{\mathrm{col}}(q) \leq \sum_{\substack{\alpha_{1}, \alpha_{2}, \alpha_{3} \in\{0,1\}}} \operatorname{Pr}\left(\operatorname{col}_{\alpha_{1} \alpha_{2} \alpha_{3}}\left(\mathcal{Q}_{q}\right) \wedge \neg \operatorname{aux}\left(\mathcal{Q}_{q}\right)\right)+\operatorname{Pr}\left(\operatorname{aux}\left(\mathcal{Q}_{q}\right)\right)+\frac{B^{2} q(q+1)}{2^{n}-B q}
$$


The probabilities $\operatorname{Pr}\left(\operatorname{col}_{\alpha_{1} \alpha_{2} \alpha_{3}}\left(\mathcal{Q}_{q}\right) \wedge \neg \operatorname{aux}\left(\mathcal{Q}_{q}\right)\right)$, for all possible $\alpha_{1} \alpha_{2} \alpha_{3}$, are bounded in Lems. 446. For $\operatorname{Pr}\left(\operatorname{aux}\left(\mathcal{Q}_{q}\right)\right)$, note that

$$
\begin{aligned}
\operatorname{Pr}\left(\operatorname{aux}\left(\mathcal{Q}_{q}\right)\right) \leq & \operatorname{Pr}\left(\operatorname{aux}_{1}\left(\mathcal{Q}_{q}\right)\right)+\operatorname{Pr}\left(\operatorname{aux}_{2}\left(\mathcal{Q}_{q}\right) \mid \neg \operatorname{aux}_{1}\left(\mathcal{Q}_{q}\right)\right)+ \\
& \sum_{i=3}^{5} \operatorname{Pr}\left(\operatorname{aux}_{i}\left(\mathcal{Q}_{q}\right) \mid \neg \operatorname{aux}_{2 \vee \cdots \vee i-1}\left(\mathcal{Q}_{q}\right)\right) .
\end{aligned}
$$

The probabilities on $\operatorname{aux}_{1}\left(\mathcal{Q}_{q}\right)$ and $\operatorname{aux}_{2}\left(\mathcal{Q}_{q}\right)$ are rather straightforward and bounded in Lem. 7. The bounds on $\operatorname{aux}_{3}\left(\mathcal{Q}_{q}\right), \ldots, \operatorname{aux}_{5}\left(\mathcal{Q}_{q}\right)$ are more involved and discussed in Lems. 8 . 10. In these lemmas, we regularly make use of Stirling's approximation: $t ! \geq(t / e)^{t}$ for any $t$. The proof ideas of these lemmas at a high level consist of noticing that any query adds a solution to the set in question with a certain maximal probability $p$, and in that case it adds at most $\tau^{\prime}$ solutions. This means that if $\tau$ solutions are required, at least $\tau / \tau^{\prime}$ queries have to contribute. Afterwards, all lemmas are combined to obtain a bound on $\mathbf{A d v}_{\mathrm{F}_{\mathrm{SS}}}^{\text {col }}(q)$.

Lemma 4. $\operatorname{Pr}\left(\operatorname{col}_{000}\left(\mathcal{Q}_{q}\right) \wedge \neg \operatorname{aux}\left(\mathcal{Q}_{q}\right)\right) \leq \frac{\left(\tau_{3}^{2}+\tau_{5}\right) B^{2} \delta_{B, C}[1] q^{2}}{2^{n}-B q}+$ $\left(\begin{array}{c}B \\ 2\end{array}\right) \frac{\left(\tau_{3}^{2} 2^{n}+\tau_{3} B^{2} q^{2}\right) \delta_{B, C}[2] q}{2^{2 n}-B q}+\left(\begin{array}{c}B \\ 2\end{array}\right) \frac{B^{4} \delta_{B, C}[2] q^{5}}{2^{3 n}-B q}$.

Proof. In this configuration, we consider (18) with an additional requirement that $x_{1} \neq x_{1}^{\prime}$, $x_{2} \neq x_{2}^{\prime}$, and $x_{3} \neq x_{3}^{\prime}$. Note that 18$)$ can be equivalently stated as

$$
\begin{aligned}
x_{1} \neq x_{1}^{\prime}, x_{2} & \neq x_{2}^{\prime}, x_{3} \neq x_{3}^{\prime}, \\
x_{1} \oplus z_{1} \oplus x_{2} \oplus z_{2} & =x_{3}, \\
x_{1}^{\prime} \oplus z_{1}^{\prime} \oplus x_{2}^{\prime} \oplus z_{2}^{\prime} & =x_{3}^{\prime}, \\
x_{2} \oplus z_{2} \oplus x_{3} \oplus z_{3} & =x_{2}^{\prime} \oplus z_{2}^{\prime} \oplus x_{3}^{\prime} \oplus z_{3}^{\prime},
\end{aligned}
$$

by XORing the second and third equations to the fourth one. We will use both representations interchangeably. For the analysis of $\operatorname{col}_{000}\left(\mathcal{Q}_{q}\right) \wedge \neg \operatorname{aux}\left(\mathcal{Q}_{q}\right)$, we say that the $i^{\text {th }}$ query $(i \in\{1, \ldots, q\})$ is successful if it makes configuration $\operatorname{col}_{000}\left(\mathcal{Q}_{i}\right)$ satisfied and $\neg$ aux $\left(\mathcal{Q}_{i}\right)$ holds. Now, by basic probability theory, we can analyze the probability of the $i^{\text {th }}$ query being successful, and sum over $i=1, \ldots, q$.

Let $i \in\{1, \ldots, q\}$. Clearly, if $\neg$ aux $\left(\mathcal{Q}_{i}\right)$ holds, the $i^{\text {th }}$ query can certainly not be successful, so we assume $\neg$ aux $\left(\mathcal{Q}_{i}\right)$ and analyze the probability the $i^{\text {th }}$ query makes $\operatorname{col}_{000}\left(\mathcal{Q}_{i}\right)$ satisfied. For regular (forward or inverse) queries, the analysis of 41 mostly carries over. The difficulty lies in the analysis of predicate queries.

Query $\overline{\boldsymbol{\pi}}_{1}\left(\boldsymbol{x}_{1}\right)$ or $\overline{\boldsymbol{\pi}}_{1}^{-1}\left(\boldsymbol{z}_{1}\right)$. The cases are the same by symmetry, and we consider $\bar{\pi}_{1}\left(x_{1}\right)$ only. Denote the query response by $z_{1}$. There are at most $B q$ choices for $\left(x_{3}^{\prime}, z_{3}^{\prime}\right)$. For any such choice, by $\neg \operatorname{aux}_{3}\left(\mathcal{Q}_{q}\right)$, there are at most $\tau_{3}$ choices for $\left(x_{1}^{\prime}, z_{1}^{\prime}\right),\left(x_{2}^{\prime}, z_{2}^{\prime}\right)$ to fulfill (23c). Again by $\neg$ aux ${ }_{3}\left(\mathcal{Q}_{q}\right)$, for any such choice there are at most $\tau_{3}$ choices for $\left(x_{2}, z_{2}\right),\left(x_{3}, z_{3}\right)$ to fulfill (23d). As $z_{1}$ is randomly drawn from a set of size at least $2^{n}-B q, 23 \mathrm{~b}$ ) is fulfilled with probability at most $\frac{\tau_{3}^{2} B q}{2^{n}-B q}$.

Query $\bar{\pi}_{2}\left(x_{2}\right)$ or $\bar{\pi}_{2}^{-1}\left(z_{2}\right)$. The cases are the same by symmetry, and we consider $\bar{\pi}_{2}\left(x_{2}\right)$ only. Denote the query response by $z_{2}$. There are at most $B q$ choices for $\left(x_{3}, z_{3}\right)$. By $\operatorname{\neg aux}_{5}\left(\mathcal{Q}_{q}\right)$, there are at most $\tau_{5}$ choices for $\left(x_{1}, z_{1}\right),\left(x_{1}^{\prime}, z_{1}^{\prime}\right),\left(x_{2}^{\prime}, z_{2}^{\prime}\right),\left(x_{3}^{\prime}, z_{3}^{\prime}\right)$ to satisfy $x_{1}^{\prime} \oplus$ $z_{1}^{\prime} \oplus x_{2}^{\prime} \oplus z_{2}^{\prime}=x_{3}^{\prime}$ and $x_{1} \oplus z_{1} \oplus x_{2}^{\prime} \oplus z_{2}^{\prime} \oplus x_{3}^{\prime} \oplus z_{3}^{\prime}=z_{3}$, hence to fulfill (18c 18d) for the particular value $z_{3}$ (where we XOR the two equations). As $z_{2}$ is randomly drawn from a set of size at least $2^{n}-B q, \sqrt{18 \mathrm{~b}}$ is fulfilled with probability at most $\frac{\tau_{5} B q}{2^{n}-B q}$.

Query $\overline{\boldsymbol{\pi}}_{\mathbf{3}}\left(\boldsymbol{x}_{\mathbf{3}}\right)$. Denote the query response by $z_{3}$. There are at most $B q$ choices for $\left(x_{3}^{\prime}, z_{3}^{\prime}\right)$. For any such choice, by $\neg$ aux $x_{3}\left(\mathcal{Q}_{q}\right)$, there are at most $\tau_{3}$ choices for $\left(x_{1}^{\prime}, z_{1}^{\prime}\right),\left(x_{2}^{\prime}, z_{2}^{\prime}\right)$ to fulfill 23c. By $\neg \operatorname{aux}_{3}\left(\mathcal{Q}_{q}\right)$, there are at most $\tau_{3}$ choices for $\left(x_{1}, z_{1}\right),\left(x_{2}, z_{2}\right)$ to fulfill $(23 \mathrm{~b})$. As $z_{3}$ 
is randomly drawn from a set of size at least $2^{n}-B q,(23 \mathrm{~d})$ is fulfilled with probability at most $\frac{\tau_{3}^{2} B q}{2^{n}-B q}$.

Query $\overline{\boldsymbol{\pi}}_{\mathbf{3}}^{-1}\left(z_{3}\right)$. Denote the query response by $x_{3}$. There are at most $B q$ choices for $\left(x_{2}, z_{2}\right)$. By $\neg \operatorname{aux}_{5}\left(\mathcal{Q}_{q}\right)$, there are at most $\tau_{5}$ choices for $\left(x_{1}, z_{1}\right),\left(x_{1}^{\prime}, z_{1}^{\prime}\right),\left(x_{2}^{\prime}, z_{2}^{\prime}\right),\left(x_{3}^{\prime}, z_{3}^{\prime}\right)$ to satisfy $x_{1}^{\prime} \oplus z_{1}^{\prime} \oplus x_{2}^{\prime} \oplus z_{2}^{\prime}=x_{3}^{\prime}$ and $x_{1} \oplus z_{1} \oplus x_{2}^{\prime} \oplus z_{2}^{\prime} \oplus x_{3}^{\prime} \oplus z_{3}^{\prime}=z_{3}$, hence to fulfill $18 \mathrm{c}-18 \mathrm{~d}$ ) for the particular value $z_{3}$ (where we XOR the two equations). As $x_{3}$ is randomly drawn from a set of size at least $2^{n}-B q, 18 \mathrm{~b}$ is fulfilled with probability at most $\frac{\tau_{5} B q}{2^{n}-B q}$.

Query $\overline{\boldsymbol{\pi}}_{\mathbf{1}}^{\boldsymbol{\Phi}}\left(\boldsymbol{y}_{\mathbf{1}}\right)$. Denote the query response by $\left\{\left(x_{1}^{1}, z_{1}^{1}\right), \ldots,\left(x_{1}^{B}, z_{1}^{B}\right)\right\}$. In case the $B$-set contributes at one position only (say $\left(x_{1}, z_{1}\right)$ ), the same reasoning as for regular queries applies with the difference that any query of the $B$-set may be successful and that the bound of Lem. 2 part (ii) applies: $\frac{\tau_{3}^{2} B^{2} \delta_{B, C}[1] q}{2^{n}-B q}$.

Now consider the case the predicate query contributes to both $\left(x_{1}, z_{1}\right)$ and $\left(x_{1}^{\prime}, z_{1}^{\prime}\right)$. There are $\left(\begin{array}{c}B \\ 2\end{array}\right)$ ways for the predicate query to contribute (or 0 if $B=1$ ). By $\neg$ aux $x_{3}\left(\mathcal{Q}_{q}\right)$, there are at most $\tau_{3}^{2} 2^{n}$ choices for $\left(x_{2}, z_{2}\right),\left(x_{2}^{\prime}, z_{2}^{\prime}\right),\left(x_{3}, z_{3}\right),\left(x_{3}^{\prime}, z_{3}^{\prime}\right)$ to fulfill $23 \mathrm{~d}$ ). By Lem. 2 part (iii), 23b 23c are fulfilled with probability at most $\left(\begin{array}{c}B \\ 2\end{array}\right) \frac{\tau_{3}^{2} \delta_{B, C}[2] 2^{n}}{2^{2 n}-B q}$.

Query $\overline{\boldsymbol{\pi}}_{2}^{\boldsymbol{\Phi}}\left(\boldsymbol{y}_{\mathbf{2}}\right)$. Denote the query response by $\left\{\left(x_{2}^{a}, z_{2}^{a}\right),\left(x_{2}^{b}, z_{2}^{b}\right)\right\}$. In case the $B$-set contributes at one position only (say $\left(x_{2}, z_{2}\right)$ ), the same reasoning as for regular queries applies with the difference that any query of the $B$-set may be successful and that the bound of Lem. 2 part (ii) applies: $\frac{\tau_{5} B^{2} \delta_{B, C}[1] q}{2^{n}-B q}$.

Now consider the case the predicate query contributes to both $\left(x_{2}, z_{2}\right)$ and $\left(x_{2}^{\prime}, z_{2}^{\prime}\right)$. There are $\left(\begin{array}{c}B \\ 2\end{array}\right)$ ways for the predicate query to contribute (or 0 if $B=1$ ). There are at most $(B q)^{2}$ choices for $\left(x_{3}, z_{3}\right),\left(x_{3}^{\prime}, z_{3}^{\prime}\right)$. By $\neg \operatorname{aux}_{3}\left(\mathcal{Q}_{q}\right)$, there are at most $\tau_{3}$ choices for $\left(x_{1}, z_{1}\right),\left(x_{1}^{\prime}, z_{1}^{\prime}\right)$ to fulfill (18d). By Lem. 2 part (iii), 18b 18c are fulfilled with probability at most $\left(\begin{array}{c}B \\ 2\end{array}\right) \frac{\tau_{3} B^{2} \delta_{B, C}[2] q^{2}}{2^{2 n}-B q}$.

Query $\overline{\boldsymbol{\pi}}_{\mathbf{3}}^{\boldsymbol{\Phi}}\left(\boldsymbol{y}_{\mathbf{3}}\right)$. Denote the query response by $\left\{\left(x_{3}^{a}, z_{3}^{a}\right),\left(x_{3}^{b}, z_{3}^{b}\right)\right\}$. In case the $B$-set contributes at one position only (say $\left(x_{3}, z_{3}\right)$ ), the same reasoning as for regular forward queries applies with the difference that any query of the $B$-set may be successful and that the bound of Lem. 2 part (ii) applies: $\frac{\tau_{3}^{2} B^{2} \delta_{B, C}[1] q}{2^{n}-B q}$.

Now consider the case the predicate query contributes to both $\left(x_{3}, z_{3}\right)$ and $\left(x_{3}^{\prime}, z_{3}^{\prime}\right)$. There are $\left(\begin{array}{c}B \\ 2\end{array}\right)$ ways for the predicate query to contribute (or 0 if $B=1$ ). There are at most $(B q)^{4}$ choices for $\left(x_{1}, z_{1}\right),\left(x_{1}^{\prime}, z_{1}^{\prime}\right),\left(x_{2}, z_{2}\right),\left(x_{2}^{\prime}, z_{2}^{\prime}\right)$. By Lem. 2 part (iv), 23b 23d are fulfilled with probability at most $\left(\begin{array}{c}B \\ 2\end{array}\right) \frac{B^{4} \delta_{B, C}[2] q^{4}}{2^{3 n}-B q}$.

Conclusion. Taking the maximum of all success probabilities, the $i^{\text {th }}$ query is successful with probability at most $\frac{\left(\tau_{3}^{2}+\tau_{5}\right) B^{2} \delta_{B, C}[1] q}{2^{n}-B q}+\left(\begin{array}{c}B \\ 2\end{array}\right) \frac{\left(\tau_{3}^{2} 2^{n}+\tau_{3} B^{2} q^{2}\right) \delta_{B, C}[2]}{2^{2 n}-B q}+\left(\begin{array}{c}B \\ 2\end{array}\right) \frac{B^{4} \delta_{B, C}[2] q^{4}}{2^{3 n}-B q}$. The claimed bound is obtained by summing over $i=1, \ldots, q$.

Lemma 5. $\operatorname{Pr}\left(\operatorname{col}_{\alpha_{1} \alpha_{2} \alpha_{3}}\left(\mathcal{Q}_{q}\right) \wedge \neg \operatorname{aux}\left(\mathcal{Q}_{q}\right)\right)=0$ for $\alpha_{1} \alpha_{2} \alpha_{3} \in\{001,010,011,101\}$, provided $\tau_{2}=1$.

Proof. We start with the cases 001,011. For these cases, 18d reduces to finding $\left(x_{1}, z_{1}\right)$, $\left(x_{1}^{\prime}, z_{1}^{\prime}\right)$ such that $x_{1} \oplus z_{1}=x_{1}^{\prime} \oplus z_{1}^{\prime}$. By $\neg$ aux $x_{2}\left(\mathcal{Q}_{q}\right)$ with $\tau_{2}=1$, there do not exist two such tuples. Similarly, for 101, in order to comply with $(18 \mathrm{~b})$ and $(18 \mathrm{c})$, it is required to find $\left(x_{2}, z_{2}\right),\left(x_{2}^{\prime}, z_{2}^{\prime}\right)$ such that $x_{2} \oplus z_{2}=x_{2}^{\prime} \oplus z_{2}^{\prime}$, and the remaining reasoning is the same. Finally for $010,\left(18 \mathrm{~b}\right.$ and (18c) can be simplified to $x_{1} \oplus z_{1} \oplus x_{3}=x_{1}^{\prime} \oplus z_{1}^{\prime} \oplus x_{3}^{\prime}$, which once XORed with (18d) reduces to finding $\left(x_{3}, z_{3}\right),\left(x_{3}^{\prime}, z_{3}^{\prime}\right)$ such that $x_{3} \oplus z_{3}=x_{3}^{\prime} \oplus z_{3}^{\prime}$, and the remaining reasoning is the same.

Lemma 6. $\operatorname{Pr}\left(\operatorname{col}_{\alpha_{1} \alpha_{2} \alpha_{3}}\left(\mathcal{Q}_{q}\right) \wedge \neg \operatorname{aux}\left(\mathcal{Q}_{q}\right)\right)=0$ for $\alpha_{1} \alpha_{2} \alpha_{3} \in\{100,110,111\}$.

Proof. If $\alpha_{1} \alpha_{2}=11$, the configuration $\operatorname{col}_{\alpha_{1} \alpha_{2} \alpha_{3}}\left(\mathcal{Q}_{q}\right)$ contradicts 1 18a), and if $\alpha_{1} \alpha_{2} \alpha_{3}=100$, it contradicts (18d). In any case, the configuration is invalid. 
Lemma 7. $\operatorname{Pr}\left(\operatorname{aux}_{1}\left(\mathcal{Q}_{q}\right)\right)+\operatorname{Pr}\left(\operatorname{aux}_{2}\left(\mathcal{Q}_{q}\right) \mid \neg \operatorname{aux}_{1}\left(\mathcal{Q}_{q}\right)\right) \leq\left(\begin{array}{c}B \\ 2\end{array}\right) \frac{\delta_{B, C}[2] q 2^{n}}{2^{2 n}-B q}+$ $2^{n-|C|}\left(\frac{e B \delta_{B, C}[1] q}{\left(\tau_{2}+1\right)\left(2^{n}-B q\right)}\right)^{\tau_{2}+1}+2^{n}\left(\frac{e B q}{\left(\tau_{2}+1\right)\left(2^{n}-B q\right)}\right)^{\tau_{2}+1}$.

Proof. We bound these probabilities separately.

$\operatorname{Pr}\left(\operatorname{aux}_{1}\left(\mathcal{Q}_{q}\right)\right)$. This auxiliary event only applies to predicate queries where $B>1$. Consider the $i^{\text {th }}$ query and let it be a predicate query $\left\{\left(\kappa_{i}, x_{i}^{1}, z_{i}^{1}\right), \ldots,\left(\kappa_{i}, x_{i}^{B}, z_{i}^{B}\right)\right\}$. Let $a, b \in$ $\{1, \ldots, B\}$ distinct. By Lem. 2 part (iii), we find that $x_{i}^{a} \oplus z_{i}^{a}=x_{i}^{b} \oplus z_{i}^{b}$ with probability at most $\frac{\delta_{B, C}[2] 2^{n}}{2^{2 n}-B q}$. Summing over all queries and all choices for $a, b$, we obtain

$$
\operatorname{Pr}\left(\operatorname{aux}_{1}\left(\mathcal{Q}_{q}\right)\right) \leq\left(\begin{array}{c}
B \\
2
\end{array}\right) \frac{\delta_{B, C}[2] q 2^{n}}{2^{2 n}-B q} .
$$

$\operatorname{Pr}\left(\operatorname{aux}_{2}\left(\mathcal{Q}_{q}\right) \mid \neg \operatorname{aux}_{1}\left(\mathcal{Q}_{q}\right)\right)$. Let $Z \in\{0,1\}^{n}$. Consider the $i^{\text {th }}$ query. If this is a regular (forward or inverse) query $\left(\kappa_{i}, x_{i}, z_{i}\right)$, it satisfies $x_{i} \oplus z_{i}=Z$ with probability at most $\frac{1}{2^{n}-B q}$. On the other hand, if it is a predicate query $\left\{\left(\kappa_{i}, x_{i}^{1}, z_{i}^{1}\right), \ldots,\left(\kappa_{i}, x_{i}^{B}, z_{i}^{B}\right)\right\}$, one of the $B$ satisfies $x_{i} \oplus z_{i}=Z$ with probability at most $\frac{B \delta_{B, C}[1]}{2^{n}-B q}$ if $\operatorname{Bits}_{C}(Z)=0$ (Lem. 2 part (ii)) and with probability at most $\frac{B}{2^{n}-B q}$ if $\operatorname{Bits}_{C}(Z) \neq 0$ (in this case the equation could never be satisfied if $B=1$, and $\delta_{B, C}[1]=1$ for $\left.B>1\right)$. By $\neg \operatorname{aux}_{1}\left(\mathcal{Q}_{q}\right)$, at most one of the $B$ queries of the tuple equals $Z$. If $\operatorname{Bits}_{C}(Z)=0$, more than $\tau_{2}$ queries result in a solution with probability at most $\left(\begin{array}{c}q \\ \tau_{2}+1\end{array}\right)\left(\frac{B \delta_{B, C}[1]}{2^{n}-B q}\right)^{\tau_{2}+1} \leq\left(\frac{e B \delta_{B, C}[1] q}{\left(\tau_{2}+1\right)\left(2^{n}-B q\right)}\right)^{\tau_{2}+1}$. If $\operatorname{Bits}_{C}(Z) \neq 0$, we similarly obtain bound $\left(\frac{e B q}{\left(\tau_{2}+1\right)\left(2^{n}-B q\right)}\right)^{\tau_{2}+1}$. Considering any possible choice for $Z$, we obtain:

$$
\begin{aligned}
\operatorname{Pr}\left(\operatorname{aux}_{2}\left(\mathcal{Q}_{q}\right) \mid \neg \operatorname{aux}_{1}\left(\mathcal{Q}_{q}\right)\right) \leq & 2^{n-|C|}\left(\frac{e B \delta_{B, C}[1] q}{\left(\tau_{2}+1\right)\left(2^{n}-B q\right)}\right)^{\tau_{2}+1}+ \\
& \left(2^{n}-2^{n-|C|}\right)\left(\frac{e B q}{\left(\tau_{2}+1\right)\left(2^{n}-B q\right)}\right)^{\tau_{2}+1} .
\end{aligned}
$$

The claim is obtained by adding (24) and a simplified version of (25).

The artificial split in the analysis of $\operatorname{Pr}\left(\operatorname{aux}_{2}\left(\mathcal{Q}_{q}\right) \mid \neg \operatorname{aux} 1\left(\mathcal{Q}_{q}\right)\right)$ is required as a special treatment is needed for $B=1$.

Lemma 8. $\operatorname{Pr}\left(\operatorname{aux}_{3}\left(\mathcal{Q}_{q}\right) \mid \neg \operatorname{aux}_{2}\left(\mathcal{Q}_{q}\right)\right) \leq$

$2^{n}\left(\frac{e\left(\tau_{2} B+\left(\begin{array}{c}B \\ 2\end{array}\right)\right) q}{\tau_{3}+1}\left(\frac{B^{2} \delta_{B, C}[1] q}{2^{n}-B q}+\frac{\delta_{B, C}[2] 2^{n}}{2^{2 n}-B q}\right)\right)^{\frac{\tau_{3}+1}{\tau_{2} B+\left(\begin{array}{c}B \\ 2\end{array}\right)}}$.

Proof. Let $Z \in\{0,1\}^{n}$. Consider the $i^{\text {th }}$ query. A special treatment is needed for predicate queries as two queries from one single $B$-set may make the entire equation satisfied.

Regular (forward or inverse) query. Denote the query by $\left(\kappa_{i}, x_{i}, z_{i}\right)$. For any other tuple $\left(\kappa_{j}, x_{j}, z_{j}\right)$ (at most $B q$ choices), the equation is satisfied with probability at most $\frac{1}{2^{n}-B q}$. Hence, the query is successful with probability at most $\frac{B q}{2^{n}-B q}$. By $\neg \operatorname{aux}_{2}\left(\mathcal{Q}_{q}\right)$, each hit adds at most $\tau_{2}$ solutions.

Predicate query. Denote the query response by $\left\{\left(\kappa_{i}, x_{i}^{1}, z_{i}^{1}\right), \ldots,\left(\kappa_{i}, x_{i}^{B}, z_{i}^{B}\right)\right\}$. In case the $B$-set contributes at one position only (say $\left(\kappa_{i}, x_{i}, z_{i}\right)$ ), the same reasoning as for regular queries applies with the difference that any query of the $B$-set may be successful and that the bound of Lem. 2 part (ii) applies: $\frac{B^{2} \delta_{B, C}[1] q}{2^{n}-B q}$. Again, by $\neg \operatorname{aux}_{2}\left(\mathcal{Q}_{q}\right)$, each hit adds at most $\tau_{2} B$ solutions. 
Now consider the case the predicate query contributes to both $\left(\kappa_{i}, x_{i}, z_{i}\right)$ and $\left(\kappa_{j}, x_{j}, z_{j}\right)$. By Lem. 2] part (iii), we find that the equation is satisfied with probability at most $\frac{\delta_{B, C}[2] 2^{n}}{2^{2 n}-B q}$, and it adds at most $\left(\begin{array}{c}B \\ 2\end{array}\right)$ solutions.

Conclusion. Thus, any query results in a solution with probability at most $\frac{B^{2} \delta_{B, C}[1] q}{2^{n}-B q}+$ $\frac{\delta_{B, C}[2] 2^{n}}{2^{2 n}-B q}$, and each hit adds at most $\tau_{2} B+\left(\begin{array}{c}B \\ 2\end{array}\right)$ solutions. Consequently, more than $\tau_{3}$ solutions are obtained with probability at most

$$
\begin{aligned}
& \left(\begin{array}{c}
q \\
\frac{\tau_{3}+1}{\tau_{2} B+\left(\begin{array}{c}
B \\
2
\end{array}\right)}
\end{array}\right)\left(\frac{B^{2} \delta_{B, C}[1] q}{2^{n}-B q}+\frac{\delta_{B, C}[2] 2^{n}}{2^{2 n}-B q}\right)^{\frac{\tau_{3}+1}{\tau_{2} B+\left(\begin{array}{c}
B \\
2
\end{array}\right)}} \\
\leq & \left(\frac{e\left(\tau_{2} B+\left(\begin{array}{c}
B \\
2
\end{array}\right)\right) q}{\tau_{3}+1}\left(\frac{B^{2} \delta_{B, C}[1] q}{2^{n}-B q}+\frac{\delta_{B, C}[2] 2^{n}}{2^{2 n}-B q}\right)\right)^{\frac{\tau_{3}+1}{\tau_{2} B+\left(\begin{array}{c}
B \\
2
\end{array}\right)}} .
\end{aligned}
$$

Considering any possible choice for $Z$, we obtain the claimed bound.

Lemma 9. $\operatorname{Pr}\left(\operatorname{aux}_{4}\left(\mathcal{Q}_{q}\right) \mid \neg \operatorname{aux}_{2 \vee 3}\left(\mathcal{Q}_{q}\right)\right) \leq 2^{2 n}\left(\frac{e \tau_{2} \tau_{3} B^{3} \delta_{B, C}[1] q^{2}}{\left(\tau_{4}+1\right)\left(2^{n}-B q\right)}\right)^{\frac{\tau_{4}+1}{\tau_{3} B}}$.

Proof. Let $Z, Z^{\prime} \in\{0,1\}^{n}$. The goal is to find tuples $\left(1, x_{i}, z_{i}\right),\left(2, x_{k}, z_{k}\right),\left(3, x_{l}, z_{l}\right) \in \mathcal{Q}_{q}$ such that

$$
\begin{aligned}
x_{k} \oplus z_{k} \oplus x_{l} & =Z^{\prime}, \\
x_{i} \oplus z_{i} \oplus x_{k} \oplus z_{k} \oplus x_{l} \oplus z_{l} & =Z .
\end{aligned}
$$

Consider the $i^{\text {th }}$ query.

Regular (forward or inverse) query to $\overline{\boldsymbol{\pi}}_{\mathbf{1}}$. Without loss of generality, consider a forward query. Denote the query by $\left(1, x_{i}, z_{i}\right)$. There are at most $B q$ choices for $\left(3, x_{l}, z_{l}\right)$. For any such choice, by $\neg \operatorname{aux}_{2}\left(\mathcal{Q}_{q}\right)$, there are at most $\tau_{2}$ choices for $\left(2, x_{k}, z_{k}\right)$ to fulfill (26a). As $z_{i}$ is randomly drawn from a set of size at least $2^{n}-B q, \sqrt{26 \mathrm{~b}}$ is fulfilled with probability at most $\frac{\tau_{2} B q}{2^{n}-B q}$. By $\neg \operatorname{aux}_{3}\left(\mathcal{Q}_{q}\right)$, each hit adds at most $\tau_{3}$ solutions (for fixed $\left(1, x_{i}, z_{i}\right)$, there are at most $\tau_{3}$ choices for $\left(2, x_{k}, z_{k}\right),\left(3, x_{l}, z_{l}\right)$ to satisfy $(26 \mathrm{~b})$ ).

Regular (forward or inverse) query to $\overline{\boldsymbol{\pi}}_{2}$. Without loss of generality, consider a forward query. Denote the query by $\left(2, x_{k}, z_{k}\right)$. There are at most $B q$ choices for $\left(3, x_{l}, z_{l}\right)$. As $z_{k}$ is randomly drawn from a set of size at least $2^{n}-B q, 26 \mathrm{a}$ is fulfilled with probability at most $\frac{B q}{2^{n}-B q}$. Again, by $\neg \operatorname{aux}_{3}\left(\mathcal{Q}_{q}\right)$, each hit adds at most $\tau_{3}$ solutions.

Regular (forward or inverse) query to $\bar{\pi}_{3}$. Consider 26) with (26a) added to (26b). Without loss of generality, consider a forward query. Denote the query by $\left(3, x_{l}, z_{l}\right)$. There are at most $B q$ choices for $\left(1, x_{i}, z_{i}\right)$. As $z_{l}$ is randomly drawn from a set of size at least $2^{n}-B q$, the replaced $26 \mathrm{~b}$ is fulfilled with probability at most $\frac{B q}{2^{n}-B q}$. Again, by $\neg$ aux ${ }_{3}\left(\mathcal{Q}_{q}\right)$, each hit adds at most $\tau_{3}$ solutions.

Predicate query. The same reasoning as for regular queries applies with the difference that any query of the $B$-set may be successful and that the bound of Lem. 2 part (ii) applies: $\frac{\tau_{2} B^{2} \delta_{B, C}[1] q}{2^{n}-B q}$. Again, by $\neg \operatorname{aux}_{3}\left(\mathcal{Q}_{q}\right)$, each hit adds at most $\tau_{3} B$ solutions.

Conclusion. Thus, any query results in a solution with probability at most $\frac{\tau_{2} B^{2} \delta_{B, C}[1] q}{2^{n}-B q}$, and each hit adds at most $\tau_{3} B$ solutions. Consequently, more than $\tau_{4}$ solutions are obtained with probability at most $\left(\begin{array}{c}q \\ \frac{\tau_{4}+1}{\tau_{3} B}\end{array}\right)\left(\frac{\tau_{2} B^{2} \delta_{B, C}[1] q}{2^{n}-B q}\right)^{\frac{\tau_{4}+1}{\tau_{3} B}} \leq\left(\frac{e \tau_{2} \tau_{3} B^{3} \delta_{B, C}[1] q^{2}}{\left(\tau_{4}+1\right)\left(2^{n}-B q\right)}\right)^{\frac{\tau_{4}+1}{\tau_{3} B}}$. Considering any possible choice for $Z, Z^{\prime}$, we obtain the claimed bound.

Lemma 10. $\operatorname{Pr}\left(\operatorname{aux}_{5}\left(\mathcal{Q}_{q}\right) \mid \neg \operatorname{aux}_{2 \vee 3 \vee}\left(\mathcal{Q}_{q}\right)\right) \leq$ $2^{n}\left(\frac{e\left(\tau_{3}\left(\begin{array}{c}B \\ 2\end{array}\right)+\tau_{2} \tau_{3} B+\tau_{4} B\right) q}{\tau_{5}+1}\left(\frac{2 \tau_{3} B^{2} \delta_{B, C}[1] q}{2^{n}-B q}+\frac{B^{2} \delta_{B, C}[2] q^{2}}{2^{2 n}-B q}\right)\right)^{\frac{\tau_{5}+1}{\tau_{3}\left(\begin{array}{c}B \\ 2\end{array}\right)+\tau_{2} \tau_{3} B+\tau_{4} B}}$. 
Proof. Let $Z \in\{0,1\}^{n}$. The goal is to find distinct tuples $\left(1, x_{i}, z_{i}\right),\left(1, x_{j}, z_{j}\right),\left(2, x_{k}, z_{k}\right)$, $\left(3, x_{l}, z_{l}\right) \in \mathcal{Q}_{q}$ such that

$$
\begin{array}{r}
x_{j} \oplus z_{j} \oplus x_{k} \oplus z_{k} \oplus x_{l}=0, \\
x_{i} \oplus z_{i} \oplus x_{k} \oplus z_{k} \oplus x_{l} \oplus z_{l}=Z .
\end{array}
$$

Consider the $i^{\text {th }}$ query. A special treatment is needed for predicate queries to $\bar{\pi}_{1}$ as it may contribute two queries to one solution.

Regular (forward or inverse) query to $\bar{\pi}_{1}$. Without loss of generality, consider a forward query. Note that it may contribute as tuple $\left(1, x_{i}, z_{i}\right)$ or as tuple $\left(1, x_{j}, z_{j}\right)$ (but not both).

First consider it a contribution to $\left(1, x_{i}, z_{i}\right)$. There are at most $B q$ choices for $\left(3, x_{l}, z_{l}\right)$. For any such choice, by $\neg \operatorname{aux}_{3}\left(\mathcal{Q}_{q}\right)$, there are at most $\tau_{3}$ choices for $\left(1, x_{j}, z_{j}\right),\left(2, x_{k}, z_{k}\right)$ to fulfill (27a). As $z_{i}$ is randomly drawn from a set of size at least $2^{n}-B q,(27 \mathrm{~b})$ is fulfilled with probability at most $\frac{\tau_{3} B q}{2^{n}-B q}$. By $\neg \operatorname{aux}_{2 \vee 3}\left(\mathcal{Q}_{q}\right)$, each hit adds at most $\tau_{2} \tau_{3}$ solutions (for fixed $\left(1, x_{i}, z_{i}\right)$, there are at most $\tau_{3}$ choices for $\left(2, x_{k}, z_{k}\right),\left(3, x_{l}, z_{l}\right)$ to satisfy $27 \mathrm{~b}$ and at most $\tau_{2}$ choices $\left(1, x_{j}, z_{j}\right)$ to then satisfy (27a)).

Next, consider it a contribution to $\left(1, x_{j}, z_{j}\right)$. There are at most $B q$ choices for $\left(3, x_{l}, z_{l}\right)$ For any such choice, by $\neg \operatorname{aux}_{3}\left(\mathcal{Q}_{q}\right)$, there are at most $\tau_{3}$ choices for $\left(1, x_{i}, z_{i}\right),\left(2, x_{k}, z_{k}\right)$ to fulfill (27b). As $z_{j}$ is randomly drawn from a set of size at least $2^{n}-B q$, 27a is fulfilled with probability at most $\frac{\tau_{3} B q}{2^{n}-B q}$. By $\neg \operatorname{aux}_{4}\left(\mathcal{Q}_{q}\right)$, each hit adds at most $\tau_{4}$ solutions (for fixed $\left(1, x_{j}, z_{j}\right)$, there are at most $\tau_{4}$ choices for $\left(1, x_{i}, z_{i}\right),\left(2, x_{k}, z_{k}\right),\left(3, x_{l}, z_{l}\right)$ to satisfy (27)).

In total, the query is successful with probability at most $\frac{2 \tau_{3} B q}{2^{n}-B q}$ and each hit adds at most $\tau_{2} \tau_{3}+\tau_{4}$ solutions.

Regular (forward or inverse) query to $\bar{\pi}_{2}$. Consider (27) with 27a added to (27b). Without loss of generality, consider a forward query. Denote the query by $\left(2, x_{k}, z_{k}\right)$. There are at most $B q$ choices for $\left(3, x_{l}, z_{l}\right)$. For any such choice, by $\neg \operatorname{aux}_{3}\left(\mathcal{Q}_{q}\right)$, there are at most $\tau_{3}$ choices for $\left(1, x_{i}, z_{i}\right),\left(1, x_{j}, z_{j}\right)$ to fulfill the replaced $(27 \mathrm{~b})$. As $z_{k}$ is randomly drawn from a set of size at least $2^{n}-B q, \sqrt{27 a}$ is fulfilled with probability at most $\frac{\tau_{3} B q}{2^{n}-B q}$. Again, by $\neg \operatorname{aux}_{2 \vee 3}\left(\mathcal{Q}_{q}\right)$, each hit adds at most $\tau_{2} \tau_{3}$ solutions.

Regular (forward or inverse) query to $\bar{\pi}_{3}$. Consider 27) with 27a added to 27b). Without loss of generality, consider a forward query. Denote the query by $\left(3, x_{l}, z_{l}\right)$. There are at most $B q$ choices for $\left(1, x_{i}, z_{i}\right)$. By $\neg \operatorname{aux}_{3}\left(\mathcal{Q}_{q}\right)$, there are at most $\tau_{3}$ choices for $\left(1, x_{j}, z_{j}\right),\left(2, x_{k}, z_{k}\right)$ to fulfill 27a. As $z_{l}$ is randomly drawn from a set of size at least $2^{n}-B q, 227$ a is fulfilled with probability at most $\frac{\tau_{3} B q}{2^{n}-B q}$. Again, by $\neg \operatorname{aux}_{2 \vee 3}\left(\mathcal{Q}_{q}\right)$, each hit adds at most $\tau_{2} \tau_{3}$ solutions.

Predicate query to $\overline{\boldsymbol{\pi}}_{1}$. Denote the query response by $\left\{\left(x_{i}^{1}, z_{i}^{1}\right), \ldots,\left(x_{i}^{B}, z_{i}^{B}\right)\right\}$. In case the $B$-set contributes at one position only (either $\left(1, x_{i}, z_{i}\right)$ or $\left.\left(1, x_{j}, z_{j}\right)\right)$, the same reasoning as for regular queries applies with the difference that any query of the $B$-set may be successful and that the bound of Lem.2 2 part (ii) applies: $\frac{2 \tau_{3} B^{2} \delta_{B, C}[1] q}{2^{n}-B q}$. Again, by $\neg \operatorname{aux}_{2 \vee 3 \vee}\left(\mathcal{Q}_{q}\right)$, each hit adds at most $\tau_{2} \tau_{3} B+\tau_{4} B$ solutions.

Now consider the case the predicate query contributes to both $\left(1, x_{i}, z_{i}\right)$ and $\left(1, x_{j}, z_{j}\right)$. There are at most $(B q)^{2}$ choices for $\left(2, x_{k}, z_{k}\right),\left(3, x_{l}, z_{l}\right)$. By Lem. 2 part (iii), we find that 27 is fulfilled with probability at most $\frac{B^{2} \delta_{B, C}[2] q^{2}}{2^{2 n}-B q}$. By $\neg \operatorname{aux}_{3}\left(\mathcal{Q}_{q}\right)$, each hit adds at most $\tau_{3}\left(\begin{array}{c}B \\ 2\end{array}\right)$ solutions.

Predicate query to $\bar{\pi}_{2}$ or $\bar{\pi}_{3}$. The same reasoning as for regular queries applies with the difference that any query of the $B$-set may be successful and that the bound of Lem. 2 part (ii) applies: $\frac{\tau_{3} B^{2} \delta_{B, C}[1] q}{2^{n}-B q}$. Again, by $\neg \operatorname{aux}_{2 \vee 3}\left(\mathcal{Q}_{q}\right)$, each hit adds at most $\tau_{2} \tau_{3} B$ solutions.

Conclusion. Thus, any query results in a solution with probability at most $\frac{2 \tau_{3} B^{2} \delta_{B, C}[1] q}{2^{n}-B q}+$ $\frac{B^{2} \delta_{B, C}[2] q^{2}}{2^{2 n}-B q}$, and each hit adds at most $\tau_{3}\left(\begin{array}{c}B \\ 2\end{array}\right)+\tau_{2} \tau_{3} B+\tau_{4} B$ solutions. Consequently, more 
than $\tau_{5}$ solutions are obtained with probability at most

$$
\begin{aligned}
& \left(\begin{array}{c}
q \\
\frac{\tau_{5}+1}{\tau_{3}\left(\begin{array}{c}
B \\
2
\end{array}\right)+\tau_{2} \tau_{3} B+\tau_{4} B}
\end{array}\right)\left(\frac{2 \tau_{3} B^{2} \delta_{B, C}[1] q}{2^{n}-B q}+\frac{B^{2} \delta_{B, C}[2] q^{2}}{2^{2 n}-B q}\right)^{\frac{\tau_{5}+1}{\tau_{3}\left(\begin{array}{c}
B \\
2
\end{array}\right)+\tau_{2} \tau_{3} B+\tau_{4} B}} \\
\leq & \left(\frac{e\left(\tau_{3}\left(\begin{array}{c}
B \\
2
\end{array}\right)+\tau_{2} \tau_{3} B+\tau_{4} B\right) q}{\tau_{5}+1}\left(\frac{2 \tau_{3} B^{2} \delta_{B, C}[1] q}{2^{n}-B q}+\frac{B^{2} \delta_{B, C}[2] q^{2}}{2^{2 n}-B q}\right)\right)^{\frac{\tau_{5}+1}{\tau_{3}\left(\begin{array}{c}
B \\
2
\end{array}\right)+\tau_{2} \tau_{3} B+\tau_{4} B}} .
\end{aligned}
$$

Considering any possible choice for $Z$, we obtain the claimed bound.

From (21), 222), and the results of Lems. 410 we find (recall that in Lem. 5 we require $\left.\tau_{2}=1\right):$

$$
\begin{aligned}
\operatorname{Adv}_{\mathrm{F}_{\mathrm{SS}}}^{\mathrm{col}}(q) \leq & \frac{\left(\tau_{3}^{2}+\tau_{5}\right) B^{2} \delta_{B, C}[1] q^{2}+B^{2} q(q+1)}{2^{n}-B q}+\frac{e^{2} B^{2}\left(\delta_{B, C}[1]\right)^{2} q^{2} 2^{n-|C|}+e^{2} B^{2} q^{2} 2^{n}}{4\left(2^{n}-B q\right)^{2}}+ \\
& \left(\begin{array}{c}
B \\
2
\end{array}\right) \frac{\left(\tau_{3}^{2} q 2^{n}+\tau_{3} B^{2} q^{3}+q 2^{n}\right) \delta_{B, C}[2]}{2^{2 n}-B q}+\left(\begin{array}{c}
B \\
2
\end{array}\right) \frac{B^{4} \delta_{B, C}[2] q^{5}}{2^{3 n}-B q}+ \\
& 2^{n}\left(\frac{e\left(B+\left(\begin{array}{c}
B \\
2
\end{array}\right)\right) q}{\tau_{3}+1}\left(\frac{B^{2} \delta_{B, C}[1] q}{2^{n}-B q}+\frac{\delta_{B, C}[2] 2^{n}}{2^{2 n}-B q}\right)\right)^{\frac{\tau_{3}+1}{B+\left(\begin{array}{c}
B \\
2
\end{array}\right)}}+ \\
& 2^{2 n}\left(\frac{e \tau_{3} B^{3} \delta_{B, C}[1] q^{2}}{\left(\tau_{4}+1\right)\left(2^{n}-B q\right)}\right)^{\frac{\tau_{4}+1}{\tau_{3} B}}+ \\
& 2^{n}\left(\frac{e\left(\tau_{3}\left(\begin{array}{c}
B+1 \\
2
\end{array}\right)+\tau_{4} B\right) q}{\tau_{5}+1}\left(\frac{2 \tau_{3} B^{2} \delta_{B, C}[1] q}{2^{n}-B q}+\frac{B^{2} \delta_{B, C}[2] q^{2}}{2^{2 n}-B q}\right)\right)^{\frac{\tau_{5}+1}{\tau_{3}\left(\begin{array}{c}
B+1 \\
2
\end{array}\right)+\tau_{4} B}} .
\end{aligned}
$$

The proof of Prop. 7 is completed by making the following simplifications: we use the fact that $2^{n}-B q \geq 2^{n-1}$ for $B q \leq 2^{n-1}$, and put $\tau_{3}=\tau-1, \tau_{4}=\tau^{2}-\tau-1$, and $\tau_{5}=$ $2 \tau^{3}+\tau^{2}(B-1)-\tau(B+3)-1$ for some integral $\tau>0$. Additionally, we use $q+1 \leq 2 q$ for $q \geq 1$.

\section{H Tightness of the Bound of Theorem 5}

For the cases $B=1$ and $C$ arbitrary, and $B=2$ and $C$ arbitrary such that $|C|>n / 2$, we derive generic attacks that demonstrate tightness of the bounds of Thm. 5 .

Proposition $8(\boldsymbol{B}=\mathbf{1})$. Let $n \in \mathbb{N}$. Suppose $\left(\pi_{1}, \pi_{2}, \pi_{3}\right) \stackrel{\&}{\leftarrow} \mathrm{BC}\left[\Phi\left(A, 1, \varphi^{C}\right)\right](n)^{3}$. Then, $\operatorname{Adv}_{\mathrm{F}_{\mathrm{SS}}}^{\mathrm{col}}(q) \geq \frac{q^{2}}{2^{n-|C|}}$.

Proof. We construct a collision-finding adversary $\mathcal{A}$ whose goal is to find a collision where $x_{2}=x_{2}^{\prime}$. By construction, to find a collision of such form for $\mathrm{F}_{\mathrm{SS}}$ (see (8)) it suffices to find two distinct values $x_{1}, x_{1}^{\prime}$ such that $x_{1} \oplus \pi_{1}\left(x_{1}\right)=x_{1}^{\prime} \oplus \pi_{1}\left(x_{1}^{\prime}\right)$. To this end, the adversary makes predicate queries to $\pi_{1}^{\Phi}$ on input of distinct values $y$ to obtain $q$ queries $\left(x_{y}, z_{y}\right)$ satisfying $\operatorname{Bits}_{C}\left(x_{y} \oplus z_{y}\right)=0$. Any two such queries collide on the entire state, $x_{y} \oplus z_{y}=x_{y^{\prime}} \oplus z_{y^{\prime}}$, with probability at least $\frac{q^{2}}{2^{n-|C|}}$.

Proposition $9(\boldsymbol{B}=\mathbf{2}$ and $|\boldsymbol{C}|>\boldsymbol{n} / \mathbf{2})$. Let $n \in \mathbb{N}$. Suppose $\left(\pi_{1}, \pi_{2}, \pi_{3}\right) \stackrel{\$}{\leftarrow}$ $\operatorname{BC}\left[\Phi\left(A, 2, \varphi^{C}\right)\right](n)^{3}$. Then, $\operatorname{Adv}_{\mathrm{F}_{\mathrm{SS}}}^{\mathrm{col}}(q) \geq \frac{q}{2^{n-|C|}}$.

Proof. We construct a collision-finding adversary $\mathcal{A}$ whose goal is to find a collision where $x_{2}=x_{2}^{\prime}$. By construction, to find a collision of such form for $\mathrm{F}_{\mathrm{SS}}$ (see (8)) it suffices to find two distinct values $x_{1}, x_{1}^{\prime}$ such that $x_{1} \oplus \pi_{1}\left(x_{1}\right)=x_{1}^{\prime} \oplus \pi_{1}\left(x_{1}^{\prime}\right)$. To this end, the adversary makes 
predicate queries to $\pi_{1}^{\Phi}$ on input of distinct values $y$ to obtain $q$ 2-sets $\left\{\left(x_{y}^{1}, z_{y}^{1}\right),\left(x_{y}^{2}, z_{y}^{2}\right)\right\}$ satisfying $\operatorname{Bits}_{C}\left(x_{y}^{1} \oplus z_{y}^{1}\right)=\operatorname{Bits}_{C}\left(x_{y}^{2} \oplus z_{y}^{2}\right)$. These two queries collide on the entire state, $x_{y}^{1} \oplus z_{y}^{1}=x_{y}^{2} \oplus z_{y}^{2}$, with probability at least $\frac{1}{2^{n-|C|}}$. If the adversary makes $q$ predicate queries, we directly obtain our bound.

\section{Proof of Theorem 6}

The bulk of the proof is captured in the following proposition.

Proposition 10. Let $n \in \mathbb{N}$. Suppose $\left(\pi_{1}, \pi_{2}, \pi_{3}\right) \stackrel{\$}{\leftarrow} \mathrm{BC}\left[\Phi\left(A, B, \varphi^{C}\right)\right](n)^{3}$. Then, for $q \leq$ $2^{n-1} / B$ and any positive integral values $\tau, \tau^{\prime}, \tau^{\prime \prime}$,

$$
\begin{aligned}
& \mathbf{A d v}_{\mathrm{F}_{\mathrm{SS}}}^{\text {epre }}(q) \leq \frac{2 \tau B^{3} q^{2}+4 \tau^{\prime \prime} \tau[B=1] B 2^{|C|} q+2 \tau^{\prime} \tau B^{3} q+4 B^{2} q^{2}}{2^{n}}+\frac{2[B=1] 2^{|C|} q^{3}}{2^{2 n}}+ \\
& 2^{n-|C|}\left(\frac{2 e B \delta_{B, C}[1] q}{\tau 2^{n}}\right)^{\tau}+2^{n}\left(\frac{2 e B q}{\tau 2^{n}}\right)^{\tau}+2^{n}\left(\frac{2 e B^{2} \delta_{B, C}[1] q^{2}}{\tau^{\prime} 2^{n}}\right)^{\tau^{\prime}}+ \\
& {[B=1]\left(\frac{2 e q}{\tau^{\prime \prime} 2^{|C|}}\right)^{\tau^{\prime \prime}}+2[B=1]\left(\frac{2 e 2^{|C|} q}{2^{n}}+\frac{2 e \tau B q}{\tau^{\prime \prime} 2^{|C|}}+\frac{2 e q^{2}}{\tau^{\prime \prime} 2^{n}}\right)^{\tau^{\prime \prime}} .}
\end{aligned}
$$

The proof follows later in the appendix, but we first prove Thm. 6 using Prop. 10.

\section{Proof of Theorem 6}

(i) and (ii). We put $\tau=n, \tau^{\prime}=n 2^{|C|}$, and $\tau^{\prime \prime}=n 2^{\max \{0, n / 2-|C|\}}$. Then, for the particular choice of $B$ (with $C$ arbitrary) the bound 28 of Prop. 10 simplifies to

$$
\begin{aligned}
\operatorname{Adv}_{\mathrm{F}_{\mathrm{SS}}}^{\text {epre }}(q) \leq & \frac{2 n q^{2}+4 q^{2}+4 e q^{2}+6 n^{2} 2^{\max \{|C|, n / 2\}} q+4 e 2^{|C|} q}{2^{n}}+\frac{2 \cdot 2^{|C|} q^{3}}{2^{2 n}}+ \\
& \frac{6 e q}{2^{\max \{|C|, n / 2\}}}+2^{n-|C|}\left(\frac{2 e 2^{|C|} q}{n 2^{n}}\right)^{n}+2 \cdot 2^{n}\left(\frac{2 e q^{2}}{n 2^{n}}\right)^{n} .
\end{aligned}
$$

For $q=2^{n / 2-n \varepsilon}$ (in case $|C| \leq n / 2$ ), and for $q=2^{n-|C|-n \varepsilon}$ (in case $|C|>n / 2$ ), it is clear that all terms approach 0 for $n \rightarrow \infty$.

(iii). We put $\tau=2$ and $\tau^{\prime}=n\left(\tau^{\prime \prime}\right.$ disappears for $\left.B \geq 2\right)$. The reasoning of previous cases carries over, and we will not go into detail. Note that we have $\delta_{B, C}[1]=1$ for $B \geq 2$.

\section{Proof of Proposition 10}

We consider any adversary that has access to $\left(\pi_{1}, \pi_{2}, \pi_{3}\right) \stackrel{\$}{\leftarrow} \mathrm{BC}\left[\Phi\left(A, B, \varphi^{C}\right)\right](n)^{3}$ and makes $q$ queries. As in App. G, these queries are stored in a query history $\mathcal{Q}$ as indexed tuples of the form $\left(\kappa_{i}, x_{i}, z_{i}\right)$ for regular queries and $\left\{\left(\kappa_{i}, x_{i}^{1}, z_{i}^{1}\right), \ldots,\left(\kappa_{i}, x_{i}^{B}, z_{i}^{B}\right)\right\}$ for predicate queries. We use the same approach as in App. G. In particular, we make the same transition from $\left(\pi_{1}, \pi_{2}, \pi_{3}\right)$ to $\left(\bar{\pi}_{1}, \bar{\pi}_{2}, \bar{\pi}_{3}\right) \stackrel{\S}{\leftarrow} \overline{\mathrm{BC}}\left[\Phi\left(A, B, \varphi^{C}\right)\right](n)^{3}$.

Let $Z \in\{0,1\}^{n}$. A preimage for $Z$ corresponds to obtaining query pairs $\left(x_{1}, z_{1}\right)$ for $\bar{\pi}_{1}$, $\left(x_{2}, z_{2}\right)$ for $\bar{\pi}_{2}$, and $\left(x_{3}, z_{3}\right)$ for $\bar{\pi}_{3}$ in the query history, such that:

$$
\begin{aligned}
x_{1} \oplus z_{1} \oplus x_{2} \oplus z_{2} & =x_{3}, \\
x_{1} \oplus z_{1} \oplus z_{3} & =Z .
\end{aligned}
$$

We define this configuration by $\operatorname{pre}\left(\mathcal{Q}_{q}\right)$. This means:

$$
\mathbf{A d v}_{\mathbf{F}_{\mathrm{SS}}}^{\text {epre }}(q)=\operatorname{Pr}\left(\operatorname{pre}\left(\mathcal{Q}_{q}\right)\right)+\frac{B^{2} q(q+1)}{2^{n}-B q},
$$


where the second part of the bound comes from the transition from WCM to AWCM.

As before, for the analysis of $\operatorname{Pr}\left(\operatorname{pre}\left(\mathcal{Q}_{q}\right)\right)$ we introduce an auxiliary event aux $\left(\mathcal{Q}_{q}\right)$. Let $\tau_{1}, \ldots, \tau_{5}>0$ be any integral values. We define $\operatorname{aux}\left(\mathcal{Q}_{q}\right)=\operatorname{aux}_{1 \vee \cdots \vee 5}\left(\mathcal{Q}_{q}\right)$, where

$$
\begin{aligned}
& \operatorname{aux}_{1}\left(\mathcal{Q}_{q}\right):\left|\left\{\left(\kappa_{i}, x_{i}, z_{i}\right) \in \mathcal{Q}_{q} \mid B=1 \wedge \operatorname{Bits}_{C}\left(x_{i}\right)=0 \wedge \operatorname{Bits}_{C}\left(z_{i}\right)=Z\right\}\right|>\tau_{1} ; \\
& \operatorname{aux}_{2}\left(\mathcal{Q}_{q}\right): \max _{Z \in\{0,1\}^{n}}\left|\left\{\left(\kappa_{i}, x_{i}, z_{i}\right) \in \mathcal{Q}_{q} \mid x_{i} \oplus z_{i}=Z\right\}\right|>\tau_{2} ; \\
& \operatorname{aux}_{3}\left(\mathcal{Q}_{q}\right): \max _{Z \in\{0,1\}^{n}}\left|\left\{\begin{array}{c}
\left(\kappa_{i}, x_{i}, z_{i}\right),\left(\kappa_{j}, x_{j}, z_{j}\right) \in \mathcal{Q}_{q} \mid \\
\kappa_{i} \neq \kappa_{j} \wedge x_{i} \oplus z_{i} \oplus x_{j} \oplus z_{j}=Z
\end{array}\right\}\right|>\tau_{3} ; \\
& \operatorname{aux}_{4}\left(\mathcal{Q}_{q}\right):\left|\left\{\begin{array}{c}
\left(2, x_{i}, z_{i}\right),\left(3, x_{j}, z_{j}\right) \in \mathcal{Q}_{q} \mid \\
B=1 \wedge x_{i} \oplus z_{i} \oplus x_{j} \oplus z_{j}=Z \wedge \operatorname{Bits}_{C}\left(x_{i} \oplus z_{i} \oplus x_{j}\right)=0
\end{array}\right\}\right|>\tau_{4} ; \\
& \operatorname{aux}_{5}\left(\mathcal{Q}_{q}\right):\left|\left\{\begin{array}{c}
\left(1, x_{i}, z_{i}\right),\left(3, x_{j}, z_{j}\right) \in \mathcal{Q}_{q} \mid \\
B=1 \wedge x_{i} \oplus z_{i} \oplus z_{j}=Z \wedge \operatorname{Bits}_{C}\left(x_{i} \oplus z_{i} \oplus x_{j}\right)=0
\end{array}\right\}\right|>\tau_{5} .
\end{aligned}
$$

Here, $\operatorname{aux}_{2 \vee 3}\left(\mathcal{Q}_{q}\right)$ is similar to $\operatorname{aux}_{2 \vee 3}\left(\mathcal{Q}_{q}\right)$ of App. G Events aux ${ }_{1 \vee 4 \vee 5}\left(\mathcal{Q}_{q}\right)$ are only employed when $B=1$ as a special treatment is needed for this case, and they only apply to $Z$ being the target range value. Again, by basic probability theory, we obtain for (30):

$$
\operatorname{Pr}\left(\operatorname{pre}\left(\mathcal{Q}_{q}\right)\right) \leq \operatorname{Pr}\left(\operatorname{pre}\left(\mathcal{Q}_{q}\right) \wedge \neg \operatorname{aux}\left(\mathcal{Q}_{q}\right)\right)+\operatorname{Pr}\left(\operatorname{aux}\left(\mathcal{Q}_{q}\right)\right) .
$$

We consider $\operatorname{Pr}\left(\operatorname{pre}\left(\mathcal{Q}_{q}\right) \wedge \neg \operatorname{aux}\left(\mathcal{Q}_{q}\right)\right)$ in Lem. 11. For $\operatorname{Pr}\left(\operatorname{aux}\left(\mathcal{Q}_{q}\right)\right)$, note that

$$
\operatorname{Pr}\left(\operatorname{aux}\left(\mathcal{Q}_{q}\right)\right) \leq \sum_{i=1}^{2} \operatorname{Pr}\left(\operatorname{aux}_{i}\left(\mathcal{Q}_{q}\right)\right)+\sum_{i=3}^{5} \operatorname{Pr}\left(\operatorname{aux}_{i}\left(\mathcal{Q}_{q}\right) \mid \neg \operatorname{aux}_{1 \vee 2}\left(\mathcal{Q}_{q}\right)\right) .
$$

The probabilities on $\operatorname{aux}_{1}\left(\mathcal{Q}_{q}\right)$, aux ${ }_{2}\left(\mathcal{Q}_{q}\right)$, and $\operatorname{aux}_{3}\left(\mathcal{Q}_{q}\right)$ are straightforward and bounded in Lem. 12. The bounds on $\operatorname{aux}_{4}\left(\mathcal{Q}_{q}\right)$ and $\operatorname{aux}_{5}\left(\mathcal{Q}_{q}\right)$ are more involved and discussed in Lem. 13 and Lem. 14 respectively. Afterwards, all lemmas are combined to obtain a bound on $\mathbf{A d v}_{\mathrm{FSS}_{\mathrm{SS}}}^{\text {epre }}(q)$.

Lemma 11. $\operatorname{Pr}\left(\operatorname{pre}\left(\mathcal{Q}_{q}\right) \wedge \neg \operatorname{aux}\left(\mathcal{Q}_{q}\right)\right) \leq$ $\frac{\left(\tau_{4}+\tau_{5}\right)[B=1] 2^{|C|} q+\tau_{2} B^{2} q^{2}+\tau_{3} B q}{2^{n}-B q}+\frac{[B=1] 2^{|C|} q^{3}}{2^{2 n}-B q}$.

Proof. Note that $(29)$ can be equivalently stated as

$$
\begin{aligned}
& x_{1} \oplus z_{1} \oplus x_{2} \oplus z_{2}=x_{3}, \\
& x_{2} \oplus z_{2} \oplus x_{3} \oplus z_{3}=Z,
\end{aligned}
$$

by XORing the two equations. We will use both representations interchangeably. For the analysis of $\operatorname{pre}\left(\mathcal{Q}_{q}\right) \wedge \neg \operatorname{aux}\left(\mathcal{Q}_{q}\right)$, we say that the $i^{\text {th }}$ query $(i \in\{1, \ldots, q\})$ is successful if it makes configuration $\operatorname{pre}\left(\mathcal{Q}_{i}\right)$ satisfied and $\neg \operatorname{aux}\left(\mathcal{Q}_{i}\right)$ holds. Now, by basic probability theory, we can analyze the probability of the $i^{\text {th }}$ query being successful, and sum over $i=1, \ldots, q$.

Let $i \in\{1, \ldots, q\}$. Clearly, if $\neg \operatorname{aux}_{i}\left(\mathcal{Q}_{i}\right)$ holds, the $i^{\text {th }}$ query can certainly not be successful, so we assume $\neg \operatorname{aux}\left(\mathcal{Q}_{i}\right)$ and analyze the probability the $i^{\text {th }}$ query makes pre $\left(\mathcal{Q}_{i}\right)$ satisfied. For regular (forward or inverse) queries, the analysis of 41 mostly carries over. The difficulty lies in the analysis of predicate queries.

Query $\bar{\pi}_{1}\left(x_{1}\right)$ or $\bar{\pi}_{1}^{-1}\left(z_{1}\right)$. The cases are the same by symmetry, and we consider $\bar{\pi}_{1}\left(x_{1}\right)$ only. Denote the query response by $z_{1}$. By $\neg \operatorname{aux}_{3}\left(\mathcal{Q}_{q}\right)$, there are at most $\tau_{3}$ choices for $\left(x_{2}, z_{2}\right),\left(x_{3}, z_{3}\right)$ to fulfill $\left.33 \mathrm{~b}\right)$. As $z_{1}$ is randomly drawn from a set of size at least $2^{n}-B q$, 33a is fulfilled with probability at most $\frac{\tau_{3}}{2^{n}-B q}$.

Query $\bar{\pi}_{2}\left(x_{2}\right)$ or $\bar{\pi}_{2}^{-1}\left(z_{2}\right)$. The cases are the same by symmetry, and we consider $\bar{\pi}_{2}\left(x_{2}\right)$ only. Denote the query response by $z_{2}$. There are at most $B q$ choices for $\left(x_{3}, z_{3}\right)$. By 
$\neg \operatorname{aux}_{2}\left(\mathcal{Q}_{q}\right)$, there are at most $\tau_{2}$ choices for $\left(x_{1}, z_{1}\right)$ to fulfill $29 \mathrm{~b}$. As $z_{2}$ is randomly drawn from a set of size at least $2^{n}-B q, 29 \mathrm{a}$ is fulfilled with probability at most $\frac{\tau_{2} B q}{2^{n}-B q}$.

Query $\overline{\boldsymbol{\pi}}_{\mathbf{3}}\left(\boldsymbol{x}_{\boldsymbol{3}}\right)$. Denote the query response by $z_{3}$. By $\neg \operatorname{aux}_{3}\left(\mathcal{Q}_{q}\right)$, there are at most $\tau_{3}$ choices for $\left(x_{1}, z_{1}\right),\left(x_{2}, z_{2}\right)$ to fulfill (33a). As $z_{3}$ is randomly drawn from a set of size at least $2^{n}-B q, 33 \mathrm{~b}$ is fulfilled with probability at most $\frac{\tau_{3}}{2^{n}-B q}$.

Query $\bar{\pi}_{3}^{-1}\left(z_{3}\right)$. Denote the query response by $x_{3}$. There are at most $B q$ choices for $\left(x_{2}, z_{2}\right)$. By $\neg \operatorname{aux}_{2}\left(\mathcal{Q}_{q}\right)$, there are at most $\tau_{2}$ choices for $\left(x_{1}, z_{1}\right)$ to fulfill $(29 \mathrm{~b})$. As $x_{3}$ is randomly drawn from a set of size at least $2^{n}-B q, 29$ a is fulfilled with probability at most $\frac{\tau_{2} B q}{2^{n}-B q}$. Query $\overline{\boldsymbol{\pi}}_{\mathbf{1}}^{\boldsymbol{\Phi}}\left(\boldsymbol{y}_{1}\right)$. Denote the query response by $\left\{\left(x_{1}^{1}, z_{1}^{1}\right), \ldots,\left(x_{1}^{B}, z_{1}^{B}\right)\right\}$. We make a distinction between $B=1$ and $B>1$. First, if $B>1$, the same reasoning as for regular queries applies with the difference that any query of the $B$-set may be successful and that the bound of Lem. 2 part (ii) applies: $[B>1] \frac{\tau_{3} B \delta_{B, C}[1]}{2^{n}-B q} \leq \frac{\tau_{3} B}{2^{n}-B q}$. Now, consider $B=1$. By $\neg \operatorname{aux}_{4}\left(\mathcal{Q}_{q}\right)$, there are at most $\tau_{4}$ choices for $\left(x_{2}, z_{2}\right),\left(x_{3}, z_{3}\right)$ to satisfy $x_{2} \oplus z_{2} \oplus x_{3} \oplus z_{3}=Z$, hence to fulfill (33b), and $\operatorname{Bits}_{C}\left(x_{2} \oplus z_{2} \oplus x_{3}\right)=0$. By Lem. 2 part (ii), the predicate query fulfills (33)

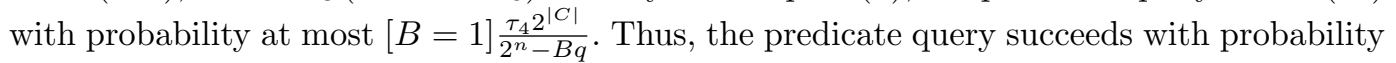
at most $\frac{\tau_{4}[B=1] 2^{|C|}+\tau_{3} B}{2^{n}-B q}$.

Query $\overline{\boldsymbol{\pi}}_{2}^{\boldsymbol{\Phi}}\left(\boldsymbol{y}_{\mathbf{2}}\right)$. Denote the query response by $\left\{\left(x_{2}^{1}, z_{2}^{1}\right), \ldots,\left(x_{2}^{B}, z_{2}^{B}\right)\right\}$. We make a distinction between $B=1$ and $B>1$. First, if $B>1$, the same reasoning as for regular queries applies with the difference that any query of the $B$-set may be successful and that the bound of Lem. 2 part (ii) applies: $[B>1] \frac{\tau_{2} B^{2} \delta_{B, C}[1] q}{2^{n}-B q} \leq \frac{\tau_{2} B^{2} q}{2^{n}-B q}$. Now, consider $B=1$. By $\neg \operatorname{aux}_{5}\left(\mathcal{Q}_{q}\right)$, there are at most $\tau_{5}$ choices for $\left(x_{1}, z_{1}\right),\left(x_{3}, z_{3}\right)$ to satisfy $x_{1} \oplus z_{1} \oplus z_{3}=Z$, hence to fulfill (29b), and $\operatorname{Bits}_{C}\left(x_{1} \oplus z_{1} \oplus x_{3}\right)=0$. By Lem. 2 part (ii), the predicate query fulfills 29 with probability at most $[B=1] \frac{\tau_{2^{n}}|C|}{2^{n}-B q}$. Thus, the predicate query succeeds with probability at most $\frac{\tau_{5}[B=1] 2^{|C|}+\tau_{2} B^{2} q}{2^{n}-B q}$.

Query $\overline{\boldsymbol{\pi}}_{\mathbf{3}}^{\boldsymbol{\Phi}}\left(\boldsymbol{y}_{\mathbf{3}}\right)$. Denote the query response by $\left\{\left(x_{3}^{1}, z_{3}^{1}\right), \ldots,\left(x_{3}^{B}, z_{3}^{B}\right)\right\}$. We make a distinction between $B=1$ and $B>1$. First, if $B>1$, the same reasoning as for regular forward queries applies with the difference that any query of the $B$-set may be successful and that the bound of Lem. 2 part (ii) applies: $[B>1] \frac{\tau_{3} B \delta_{B, C}[1]}{2^{n}-B q} \leq \frac{\tau_{3} B}{2^{n}-B q}$. Now, consider $B=1$. There are at most $q^{2}$ choices for $\left(x_{1}, z_{1}\right),\left(x_{2}, z_{2}\right)$. Similar to Lem. 2 part (iii), one can prove that the predicate query fulfills 29 with probability at most $[B=1]{\frac{2}{2^{2 n}-B q} q^{2}}^{2}$. Thus, the predicate query succeeds with probability at most $\frac{[B=1] 2^{|C|} q^{2}}{2^{2 n}-B q}+\frac{\tau_{3} B}{2^{n}-B q}$.

Conclusion. Taking the maximum of all success probabilities, the $i^{\text {th }}$ query is successful with probability at most $\frac{\left(\tau_{4}+\tau_{5}\right)[B=1] 2^{|C|}+\tau_{2} B^{2} q+\tau_{3} B}{2^{n}-B q}+\frac{[B=1] 2^{|C|} q^{2}}{2^{2 n}-B q}$. The claimed bound is obtained by summing over $i=1, \ldots, q$.

Lemma 12. $\operatorname{Pr}\left(\operatorname{aux}_{1}\left(\mathcal{Q}_{q}\right)\right)+\operatorname{Pr}\left(\operatorname{aux}_{2}\left(\mathcal{Q}_{q}\right)\right)+\operatorname{Pr}\left(\operatorname{aux}_{3}\left(\mathcal{Q}_{q}\right) \mid \neg \operatorname{aux}_{1 \vee 2}\left(\mathcal{Q}_{q}\right)\right) \leq$ $[B=1]\left(\frac{e 2^{n-|C|} q}{\left(\tau_{1}+1\right)\left(2^{n}-q\right)}\right)^{\tau_{1}+1}+2^{n-|C|}\left(\frac{e B^{2} \delta_{B, C}[1] q}{\left(\tau_{2}+1\right)\left(2^{n}-B q\right)}\right)^{\frac{\tau_{2}+1}{B}}+$ $2^{n}\left(\frac{e B^{2} q}{\left(\tau_{2}+1\right)\left(2^{n}-B q\right)}\right)^{\frac{\tau_{2}+1}{B}}+2^{n}\left(\frac{e \tau_{2} B^{3} \delta_{B, C}[1] q^{2}}{\left(\tau_{3}+1\right)\left(2^{n}-B q\right)}\right)^{\frac{\tau_{3}+1}{\tau_{2} B}}$.

Proof. We bound these probabilities separately.

$\operatorname{Pr}\left(\operatorname{aux}_{1}\left(\mathcal{Q}_{q}\right)\right)$. Recall that aux $\left(\mathcal{Q}_{q}\right)$ only applies to $B=1$. Let $Z$ be the challenge range value. Consider the $i^{\text {th }}$ query. If this is a regular (forward or inverse) query $\left(\kappa_{i}, x_{i}, z_{i}\right)$, it satisfies the condition with probability at most $\frac{2^{n-|C|}}{2^{n}-q}$. If it is a predicate query $\left(\kappa_{i}, x_{i}, z_{i}\right)$, it satisfies the condition with probability at most $\frac{2^{n-|C|}}{2^{n}-q}$ (Lem. 2 part (i)). More than $\tau_{1}$ solutions are obtained with probability at most $\left(\begin{array}{c}q \\ \tau_{1}+1\end{array}\right)\left(\frac{2^{n-|C|}}{2^{n}-q}\right)^{\tau_{1}+1} \leq\left(\frac{e 2^{n-|C|} q}{\left(\tau_{1}+1\right)\left(2^{n}-q\right)}\right)^{\tau_{1}+1}$. 
As the event only applies to the challenge value $Z$, we obtain:

$$
\operatorname{Pr}\left(\operatorname{aux}_{1}\left(\mathcal{Q}_{q}\right)\right) \leq[B=1]\left(\frac{e 2^{n-|C|} q}{\left(\tau_{1}+1\right)\left(2^{n}-q\right)}\right)^{\tau_{1}+1} .
$$

$\operatorname{Pr}\left(\operatorname{aux}_{2}\left(\mathcal{Q}_{q}\right)\right)$. The analysis differs from the one of Lem. 7 in the fact that each hit may add at most $B$ solutions (rather than 1). Taking this into account results in the following bound:

$$
\operatorname{Pr}\left(\operatorname{aux}_{2}\left(\mathcal{Q}_{q}\right)\right) \leq 2^{n-|C|}\left(\frac{e B^{2} \delta_{B, C}[1] q}{\left(\tau_{2}+1\right)\left(2^{n}-B q\right)}\right)^{\frac{\tau_{2}+1}{B}}+2^{n}\left(\frac{e B^{2} q}{\left(\tau_{2}+1\right)\left(2^{n}-B q\right)}\right)^{\frac{\tau_{2}+1}{B}} .
$$

$\operatorname{Pr}\left(\operatorname{aux}_{3}\left(\mathcal{Q}_{q}\right) \mid \neg \operatorname{aux}_{2}\left(\mathcal{Q}_{q}\right)\right)$. Event aux ${ }_{3}\left(\mathcal{Q}_{q}\right)$ differs from $\operatorname{aux}_{3}\left(\mathcal{Q}_{q}\right)$ in the fact that a single predicate query cannot make the entire equation satisfied. Leaving out this special case in the analysis of Lem. 8 results in the following bound:

$$
\operatorname{Pr}\left(\operatorname{aux}_{3}\left(\mathcal{Q}_{q}\right) \mid \neg \operatorname{aux}_{2}\left(\mathcal{Q}_{q}\right)\right) \leq 2^{n}\left(\frac{e \tau_{2} B^{3} \delta_{B, C}[1] q^{2}}{\left(\tau_{3}+1\right)\left(2^{n}-B q\right)}\right)^{\frac{\tau_{3}+1}{\tau_{2} B}} .
$$

The claim is obtained by adding 3436$)$.

Lemma 13. $\operatorname{Pr}\left(\operatorname{aux}_{4}\left(\mathcal{Q}_{q}\right) \mid \neg \operatorname{aux}_{1 \vee 2}\left(\mathcal{Q}_{q}\right)\right) \leq[B=1]\left(\frac{e \tau_{1} \tau_{2} 2^{|C|} q+e \tau_{2} q^{2}}{\left(\tau_{4}+1\right)\left(2^{n}-q\right)}\right)^{\frac{\tau_{4}+1}{\tau_{2}}}$.

Proof. Recall that $\operatorname{aux}_{4}\left(\mathcal{Q}_{q}\right)$ only applies to $B=1$. Let $Z$ be the challenge range value. The goal is to find distinct tuples $\left(2, x_{2}, z_{2}\right)$ and $\left(3, x_{3}, z_{3}\right) \in \mathcal{Q}_{q}$ such that $x_{2} \oplus z_{2} \oplus x_{3} \oplus z_{3}=Z$ and $\operatorname{Bits}_{C}\left(x_{2} \oplus z_{2} \oplus x_{3}\right)=0$. Consider the $i^{\text {th }}$ query.

Regular (forward or inverse) query. We follow the analysis of $\operatorname{aux}_{3}\left(\mathcal{Q}_{q}\right)$, only focussing on $x_{2} \oplus z_{2} \oplus x_{3} \oplus z_{3}=Z$. The query is successful with probability at most $\frac{q}{2^{n}-q}$ and each hit adds at most $\tau_{2}$ solutions.

Predicate query to $\bar{\pi}_{2}$. Denote the query response by $\left(x_{2}, z_{2}\right)$, which will satisfy $\operatorname{Bits}_{C}\left(x_{2} \oplus\right.$ $\left.z_{2}\right)=0$. By $\neg \operatorname{aux}_{1}\left(\mathcal{Q}_{q}\right)$, there are at most $\tau_{1}$ choices for $\left(x_{3}, z_{3}\right)$ to satisfy $\operatorname{Bits}_{C}\left(x_{3}\right)=0$ and $\operatorname{Bits}_{C}\left(z_{3}\right)=\operatorname{Bits}_{C}(Z)$. By Lem. 2 part (ii), we find that the equation is satisfied with probability at most $\frac{\tau_{1} 2^{|C|}}{2^{n}-q}$. By $\neg \operatorname{aux}_{2}\left(\mathcal{Q}_{q}\right)$, each hit adds at most $\tau_{2}$ solutions.

Predicate query to $\overline{\boldsymbol{\pi}}_{\mathbf{3}}$. Denote the query response by $\left(x_{3}, z_{3}\right)$, which will satisfy $\operatorname{Bits}_{C}\left(x_{3} \oplus\right.$ $\left.z_{3}\right)=0$. There are at most $q$ choices for $\left(x_{2}, z_{2}\right)$. Similar to Lem. 2 part (ii), one can prove that the equation is satisfied with probability at most $\frac{2^{n-|C|}}{2^{2^{n-|C|}-q}} \cdot q \leq \frac{q}{2^{n}-q}$ : we need the predicate query to satisfy $\operatorname{Bits}_{C}\left(x_{3}\right)=\operatorname{Bits}_{C}\left(x_{2} \oplus z_{2}\right), \operatorname{Bits}_{C}\left(x_{3} \oplus z_{3}\right)=0=\operatorname{Bits}_{C}\left(x_{2} \oplus z_{2} \oplus Z\right)$, and $\operatorname{Bits}_{\bar{C}}\left(x_{3} \oplus z_{3}\right)=\operatorname{Bits}_{\bar{C}}\left(x_{2} \oplus z_{2} \oplus Z\right)$, and this gives at most $2^{n-|C|}$ possible tuples of $\bar{\Sigma}_{k}\left(P, P^{\Phi}\right)$. By $\neg \operatorname{aux}_{2}\left(\mathcal{Q}_{q}\right)$, each hit adds at most $\tau_{2}$ solutions.

Conclusion. Thus, if $B=1$, any query results in a solution with probability at most $\frac{\tau_{1} 2^{|C|}+q}{2^{n}-q}$, and each hit adds at most $\tau_{2}$ solutions. Consequently, more than $\tau_{4}$ solutions are obtained with probability at most $\left(\begin{array}{c}q \\ \frac{\tau_{4}+1}{\tau_{2}}\end{array}\right)\left(\frac{\tau_{1} 2^{|C|}+q}{2^{n}-q}\right)^{\frac{\tau_{4}+1}{\tau_{2}}} \leq\left(\frac{e \tau_{1} \tau_{2} 2^{|C|} q+e \tau_{2} q^{2}}{\left(\tau_{4}+1\right)\left(2^{n}-q\right)}\right)^{\frac{\tau_{4}+1}{\tau_{2}}}$.

Lemma 14. $\operatorname{Pr}\left(\operatorname{aux}_{5}\left(\mathcal{Q}_{q}\right) \mid \neg \operatorname{aux}_{1 \vee 2}\left(\mathcal{Q}_{q}\right)\right) \leq$

$[B=1]\left(\frac{e \tau_{1} \tau_{2} 2^{|C|} q+e \tau_{2}^{2} 2^{n-|C|} q+e \tau_{2} q^{2}}{\left(\tau_{5}+1\right)\left(2^{n}-q\right)}\right)^{\frac{\tau_{5}+1}{\tau_{2}}}$.

Proof. Recall that $\operatorname{aux}_{5}\left(\mathcal{Q}_{q}\right)$ only applies to $B=1$. Let $Z$ be the challenge range value. The goal is to find distinct tuples $\left(1, x_{1}, z_{1}\right)$ and $\left(3, x_{3}, z_{3}\right) \in \mathcal{Q}_{q}$ such that $x_{1} \oplus z_{1} \oplus z_{3}=Z$ and $\operatorname{Bits}_{C}\left(x_{1} \oplus z_{1} \oplus x_{3}\right)=0$, or equivalently $x_{1} \oplus z_{1} \oplus z_{3}=Z$ and $\operatorname{Bits}_{C}\left(x_{3} \oplus z_{3}\right)=0$. Consider the $i^{\text {th }}$ query. 
Regular (forward or inverse) query to $\overline{\boldsymbol{\pi}}_{1}$. Without loss of generality, consider a forward query. Denote the query by $\left(x_{1}, z_{1}\right)$. There are at most $q$ choices for $\left(x_{3}, z_{3}\right)$. As $z_{1}$ is randomly drawn from a set of size at least $2^{n}-q$, the equation is satisfied with probability at most $\frac{q}{2^{n}-q}$. Each hit adds at most 1 solution.

Regular forward query to $\bar{\pi}_{3}$. By $\neg \operatorname{aux}_{2}\left(\mathcal{Q}_{q}\right)$, there are at most $\tau_{2} 2^{n-|C|}$ choices for $\left(x_{1}, z_{1}\right)$ to satisfy $\operatorname{Bits}_{C}\left(x_{1} \oplus z_{1} \oplus x_{3}\right)=0$. As $z_{3}$ is randomly drawn from a set of size at least $2^{n}-q$, the equation is satisfied with probability at most $\frac{\tau_{2} 2^{n-|C|}}{2^{n}-q}$. By $\neg \operatorname{aux}_{2}\left(\mathcal{Q}_{q}\right)$, each hit adds at most $\tau_{2}$ solutions.

Regular inverse query to $\overline{\boldsymbol{\pi}}_{\mathbf{3}}$. By $\neg \operatorname{aux}_{2}\left(\mathcal{Q}_{q}\right)$, there are at most $\tau_{2}$ choices for $\left(x_{1}, z_{1}\right)$ to satisfy $x_{1} \oplus z_{1} \oplus z_{3}=Z$. As $x_{3}$ is randomly drawn from a set of size at least $2^{n}-q$, the equation is satisfied with probability at most $\frac{\tau_{2} 2^{n-|C|}}{2^{n}-q}$. By $\neg \operatorname{aux}_{2}\left(\mathcal{Q}_{q}\right)$, each hit adds at most $\tau_{2}$ solutions.

Predicate query to $\overline{\boldsymbol{\pi}}_{\mathbf{1}}$. Denote the query response by $\left(x_{1}, z_{1}\right)$, which will satisfy $\operatorname{Bits}_{C}\left(x_{1} \oplus\right.$ $\left.z_{1}\right)=0$. By $\neg \operatorname{aux}_{1}\left(\mathcal{Q}_{q}\right)$, there are at most $\tau_{1}$ choices for $\left(x_{3}, z_{3}\right)$ to satisfy $\operatorname{Bits}_{C}\left(x_{3}\right)=0$ and $\operatorname{Bits}_{C}\left(z_{3}\right)=\operatorname{Bits}_{C}(Z)$. By Lem. 2 part (ii), we find that the equation is satisfied with probability at most $\frac{\tau_{1} 2^{|C|}}{2^{n}-q}$. Each hit adds at most 1 solution.

Predicate query to $\bar{\pi}_{3}$. Denote the query response by $\left(x_{3}, z_{3}\right)$, which will satisfy Bits $C\left(x_{3} \oplus\right.$ $\left.z_{3}\right)=0$. There are at most $q$ choices for $\left(x_{1}, z_{1}\right)$. Similar to the proof of Lem. 13, one can prove that the equation is satisfied with probability at most $\frac{2^{n-|C|}}{2^{n} 2^{n-|C|}-q} \cdot q \leq \frac{q}{2^{n}-q}$ : we need the predicate query to satisfy $\operatorname{Bits}_{C}\left(x_{3}\right)=\operatorname{Bits}_{C}\left(x_{1} \oplus z_{1}\right)$, $\operatorname{Bits}_{C}\left(x_{3} \oplus z_{3}\right)=0$, and $z_{3}=x_{1} \oplus z_{1} \oplus Z$, and this gives at most $2^{n-|C|}$ possible tuples of $\bar{\Sigma}_{k}\left(P, P^{\Phi}\right)$. By $\neg \operatorname{aux}_{2}\left(\mathcal{Q}_{q}\right)$, each hit adds at most $\tau_{2}$ solutions.

Conclusion. Thus, if $B=1$, any query results in a solution with probability at most $\frac{\tau_{1} 2^{|C|}+\tau_{2} 2^{n-|C|}+q}{2^{n}-q}$, and each hit adds at most $\tau_{2}$ solutions. Consequently, more than $\tau_{5}$ solutions are obtained with probability at most $\left(\begin{array}{c}q \\ \frac{\tau_{5}+1}{\tau_{2}}\end{array}\right)\left(\frac{\tau_{1} 2^{|C|}+\tau_{2} 2^{n-|C|}+q}{2^{n}-q}\right)^{\frac{\tau_{5}+1}{\tau_{2}}} \leq$ $\left(\frac{e \tau_{1} \tau_{2} 2^{|C|} q+e \tau_{2}^{2} 2^{n-|C|} q+e \tau_{2} q^{2}}{\left(\tau_{5}+1\right)\left(2^{n}-q\right)}\right)^{\frac{\tau_{5}+1}{\tau_{2}}}$.

From 30-31), 32, and the results of Lems. 11 14 we find:

$$
\begin{aligned}
\operatorname{Adv}_{\mathrm{F}_{\mathrm{SS}}}^{\mathrm{epre}}(q) \leq & \frac{\left(\tau_{4}+\tau_{5}\right)[B=1] 2^{|C|} q+\tau_{2} B^{2} q^{2}+\tau_{3} B q+B^{2} q(q+1)}{2^{n}-B q}+\frac{[B=1] 2^{|C|} q^{3}}{2^{2 n}-B q}+ \\
& {[B=1]\left(\frac{e 2^{n-|C|} q}{\left(\tau_{1}+1\right)\left(2^{n}-q\right)}\right)^{\tau_{1}+1}+2^{n-|C|}\left(\frac{e B^{2} \delta_{B, C}[1] q}{\left(\tau_{2}+1\right)\left(2^{n}-B q\right)}\right)^{\frac{\tau_{2}+1}{B}}+} \\
& 2^{n}\left(\frac{e B^{2} q}{\left(\tau_{2}+1\right)\left(2^{n}-B q\right)}\right)^{\frac{\tau_{2}+1}{B}}+2^{n}\left(\frac{e \tau_{2} B^{3} \delta_{B, C}[1] q^{2}}{\left(\tau_{3}+1\right)\left(2^{n}-B q\right)}\right)^{\frac{\tau_{3}+1}{\tau_{2} B}}+ \\
& {[B=1]\left(\frac{e \tau_{1} \tau_{2} 2^{|C|} q+e \tau_{2} q^{2}}{\left(\tau_{4}+1\right)\left(2^{n}-q\right)}\right)^{\frac{\tau_{4}+1}{\tau_{2}}}+} \\
& {[B=1]\left(\frac{e \tau_{1} \tau_{2} 2^{|C|} q+e \tau_{2}^{2} 2^{n-|C|} q+e \tau_{2} q^{2}}{\left(\tau_{5}+1\right)\left(2^{n}-q\right)}\right)^{\frac{\tau_{5}+1}{\tau_{2}}} . }
\end{aligned}
$$

The proof of Prop. 10 is completed by making the following simplifications: we use the fact that $2^{n}-B q \geq 2^{n-1}$ for $B q \leq 2^{n-1}$, and put $\tau_{2}=\tau B-1, \tau_{3}=\tau^{\prime} \tau_{2} B-1, \tau_{1}=\tau^{\prime \prime}-1$, and $\tau_{4}=\tau_{5}=\tau^{\prime \prime} \tau_{2}-1$ for some integral $\tau, \tau^{\prime}, \tau^{\prime \prime}>0$. Additionally, we use $q+1 \leq 2 q$ for $q \geq 1$. 